Kyoto University,

Graduate School of Economics

Discussion Paper Series

Optimality of Naive Investment Strategies in Dynamic MeanVariance Optimization Problems with Multiple Priors

Yuki Shigeta

Discussion Paper No. E-16-004

Graduate School of Economics Kyoto University

Yoshida-Hommachi, Sakyo-ku

Kyoto City, 606-8501, Japan

July, 2016 


\title{
Optimality of Naive Investment Strategies in Dynamic Mean-Variance Optimization Problems with Multiple Priors*
}

\author{
Yuki Shigeta ${ }^{\dagger}$
}

July 1, 2016

\begin{abstract}
We study dynamic mean-variance optimization problems with multiple priors. We introduce two types of multiple priors, the priors for expected returns and the priors for covariances. Our framework suggests that the global minimumvariance portfolio is optimal when the investor strongly doubts the correctness of the estimated expected returns, and the equally weighted portfolio is optimal when the investor strongly doubts the correctness of the estimated covariances. From the back tests, we find that for some data sets, the strategy that invests in the global minimum-variance portfolio or the equally weighted portfolio considering the market condition is more efficient than the other mean-variance efficient portfolios.
\end{abstract}

Key words: Robust mean-variance optimization; dynamic portfolio selections; naive diversification; global minimum-variance portfolio; mean-variance efficiency.

JEL Classification: G11

*Acknowledgements: I would like to express my gratitude to Prof. Masahiko Egami and Prof. Katsutoshi Wakai for their helpful suggestions in regard to this paper. I am also grateful to Prof. Yuji Yamada and to the participants of the 24th annual meeting of the Nihon Financial Association for their helpful advice.

${ }^{\dagger}$ Graduate School of Economics, Kyoto university, Japan. E-mail address: sy46744@gmail.com 


\section{Introduction}

The mean-variance analysis proposed by Markowitz (1952) and developed by Tobin (1958) and Merton (1972) tells us that the tangency portfolio combined with the risk-free asset is meanvariance efficient, which means that it delivers the highest Sharpe ratio among all portfolios. However, it is not always efficient in practice. For example, Jagannathan and Ma (2003) mention that the global minimum-variance portfolio delivers as large an out-of-sample Sharpe ratio as the other efficient portfolios when the estimated expected returns are used. DeMiguel, Garlappi, and Uppal (2009b) report that in their back tests, the equally weighted portfolio $(1 / N$ portfolio) often has a higher Sharpe ratio than other optimal portfolios including the tangency portfolio.

Probable explanations of these practical pitfalls are difficulties in estimating expected returns and sensitivity of the tangency portfolio to the expected returns. Merton (1980) reports that the estimation of expected returns is more difficult than that of a covariance matrix of returns. Best and Grauer (1991) show that the mean-variance efficient portfolios are very sensitive to the values of the expected returns. Therefore, the estimation errors of expected returns have a large impact on optimal portfolio selections. Consequently, in practice, the global minimum-variance portfolio, which is not mean-variance efficient in theory, and the equally weighted portfolio, which seems to be naive, can become more efficient than the mean-variance efficient portfolios.

In this paper, we construct plausible mean-variance optimization problems with multiple priors and show that in limiting cases, the solutions to these problems include the equally weighted portfolio and the global minimum-variance portfolio. The mean-variance problems with multiple priors are robust to estimation errors of moments of asset returns. We consider two-dimensional multiple priors: the priors for expected returns and the priors for a covariance matrix of returns. In our framework, the naive and inefficient strategies such as the equally weighted portfolio and the global minimum-variance portfolio are characterized by an investor's suspicion of the estimated parameters. If an investor strongly doubts the estimated expected returns, her optimal portfolio becomes similar to the global minimum-variance portfolio. In contrast to that, it becomes similar to the equally weighted portfolio if an investor strongly doubts the estimated covariances.

Moreover, our framework is based on dynamic optimization problems and allows dependency among the asset returns over time. More specifically, the market model in our framework is the Markovian market model where a Markov process affects the distribution of returns. This includes various return models such as the factor pricing models (e.g., capital asset pricing model (CAPM) by Sharpe (1964) and the Fama-French three factor model by Fama and French (1993)), the Markov regime switching models (e.g., Hamilton (1989) and Ang and Bekaert (2002)) and so on. Furthermore, the dynamic approach justifies the investment strategies which seem myopic. For example, the strategy investing in the global minimumvariance portfolio during economic booming and investing in the equally weighted portfolio during economic recession can be justified by our framework.

In order to investigate the effects of the estimation errors and study the efficiency of the optimal portfolio in our framework, we conduct back tests using various data sets. Similar to previous research, our back tests demonstrate that the global minimum-variance portfolio is sometimes more efficient than the tangency portfolio. On the other hand, the investment strategy that invests in the equally weighted portfolio during the economic booming and the global minimum-variance portfolio during the economic recession is sometimes the most efficient in all the portfolios. This strategy seems to be naive. However, since it is characterized by the limiting cases in our framework, the reason why the investor chooses it is because of the strong suspicion of the estimated parameters.

In the investment theory, the various methods have been proposed to deal with the esti- 
mation errors. They are classified into two major approaches: the Bayesian approach and the non-Bayesian approach. In this paper, we adopt the non-Bayesian approach, specifically, the max-min approach; maximizing an objective function with respect to portfolios after minimizing the objective function with respect to priors of return's distribution. The max-min problem is popular when dealing with the multiple prior optimization. Goldfarb and Iyengar (2003) solve the mean-variance portfolio selections with multiple priors under the factor pricing model. Garlappi, Uppal, and Wang (2007) characterize multiple priors for the expected returns by the confidence interval around the estimated expected returns and find that their multiple-priors optimal portfolios have high Sharpe ratios in their back tests. The max-min approach is economically axiomatized by Gilboa and Schmeidler (1989) in order to describe the ambiguity aversion of investors illustrated by Ellsberg (1961).

In addition, we use one concept from the Bayesian approach. The Bayesian approach is based on the Bayesian statistics, e.g., shrinkage estimators (Jobson and Korkie (1980) and Jorion (1986)), the Black-Litterman model (Black and Litterman (1990)) and the other methods such as Pástor and Stambaugh (2000). Ledoit and Wolf (2003) propose the shrinkage estimator to estimate a covariance matrix of asset returns when facing the small sample problem that is when the number of assets is larger than the number of observations. To compute the shrinkage estimator, they minimize the Frobenius norm of estimated errors of the covariance matrix. Using the idea of Ledoit and Wolf (2003), we introduce the Frobenius norm of estimation errors of a covariance matrix in order to deal with multiple priors.

The results obtained by the mean-variance optimization constraining portfolio norms are similar to the results by our framework. The mean-variance optimization constraining portfolio norms is proposed by DeMiguel, Garlappi, Nogales, and Uppal (2009a). Especially, their 2 -norm-constrained portfolio is basically the same as the one of our limiting cases. The differences between our model and the one in DeMiguel et al. (2009a) are investment horizon and problem formulation. DeMiguel et al. (2009a) consider static portfolio selections such as

$$
\min _{u} u^{\prime} \widehat{\Sigma} u \quad \text { subject to } u^{\prime} u \leq k,
$$

where $u$ is a portfolio vector and $u^{\prime}$ is its transpose. $\widehat{\Sigma}$ is an estimated covariance matrix. $k$ represents the upper bound of the constraint. DeMiguel et al. (2009a) solve the above static minimum-variance problem since it is free from the problem of time-inconsistency ${ }^{1}$. The optimal 2-norm-constrained portfolio is based on the covariance estimator of Ledoit and Wolf (2004). If the upper boundary of the constraint is the reciprocal of the number of assets, then the optimal portfolio becomes the equally weighted portfolio.

In contrast to the above problem, we consider the mean-variance-style objective function such that

$$
\mathrm{E}^{\theta, V}\left[\sum_{t=0}^{T-1} \delta^{t}\left(\mathrm{E}^{\theta, V}\left[X_{t+1} \mid \mathcal{F}_{t}^{R, F}\right]-\frac{\widehat{\gamma}_{t}}{2 X_{t}} \operatorname{Var}^{\theta, V}\left(X_{t+1} \mid \mathcal{F}_{t}^{R, F}\right)\right)\right],
$$

where $X_{t}$ is a wealth of the investor at time $t$ and $\mathcal{F}_{t}^{R, F}$ is the information of the investor at time $t . \widehat{\gamma}_{t} / X_{t}$ represents the trade-off between the expected return and associated risks. The superscripts $\theta$ and $V$ express multiple priors and $\delta$ is a constant discount rate. Hence, this objective function is an expected value of discounted sum of objectives in conditional mean-variance problems. The above objective function is proposed by Chen, $\mathrm{Li}$, and Zhao (2014) to avoid the problem of time-inconsistency. Chen et al. (2014) consider this objective function with a unique prior, but we consider the objective function with multiple priors.

Although there are several differences, the results of Pflug, Pichler, and Wozabal (2012) are essentially the same as our results. Pflug et al. (2012) characterize a degree of ambiguity

\footnotetext{
${ }^{1}$ The standard dynamic programming procedure can not be applied to the simple mean-variance optimization. See $\mathrm{Li}$ and $\mathrm{Ng}$ (2000) for more details.
} 
as the Kantorovich metric, and show that for various objective functions including a meanstandard-deviation objective function and for arbitrary distributions of asset returns, the investor chooses the equally weighted portfolio under high model ambiguity. In contrast to Pflug et al. (2012), we focus on mean-variance objective functions and Markovian return models. This allows us to use more simple and clear degrees of ambiguity than the Kantorovich metric, that is, we use a confidence interval around the estimated expected return vector and a relative error of the estimated covariance matrix. Furthermore, our optimization problems are dynamic optimization problems, whereas optimization problems in Pflug et al. (2012) are static optimization problems. Therefore, our models have different implications from the model in Pflug et al. (2012).

The rest of this paper is organized as follows. Section 2 formulates and solves the dynamic mean-variance optimization problem with multiple priors. Section 3 investigates limiting behaviors of the solutions. Section 4 conducts back tests and reports their results. Section 5 is the concluding section. The most proofs of theorems, lemmas and propositions in this paper are in Appendix.

\section{Mean-Variance Portfolio Selections with Multiple Priors in a Markovian Market}

First, we introduce the following notations.

- $\mathbb{R}^{d}$, the $d$-dimensional Euclidean space. Specifically, we write $\mathbb{R}:=\mathbb{R}^{1}$ and $\mathbb{R}_{+}:=\{x \in$ $\mathbb{R} \mid x \geq 0\}$.

- $\mathbb{R}^{m, n}$, a set of $m \times n$ matrices.

- $x^{\prime}$, the transpose of a vector or matrix $x$.

- $\mathbf{0}_{d}$, the $d$-dimensional real-valued vector whose all elements are 0 .

- $\mathbf{1}_{d}$, the $d$-dimensional real-valued vector whose all elements are 1 .

- $I_{d}$, the $d$-dimensional identity matrix.

- $\mathbb{1}_{A}(\omega)$, an indicator function. If $\omega \in A$, then $\mathbb{1}_{A}(\omega)=1$. If $\omega \notin A$, then $\mathbb{1}_{A}(\omega)=0$.

- $(x)^{+}:=\max \{x, 0\}$ for all $x \in \mathbb{R}$.

- $\|x\|$, a general expression of norms. Specifically, $\|x\|$ is the Euclidean norm of $x \in \mathbb{R}^{d}$ and $\|A\|$ is the Frobenius norm of $A \in \mathbb{R}^{m \times n}$.

Let $(\Omega, \mathcal{F}, \mathbb{P})$ be a probability space. We consider optimal portfolio selections of an investor during $T$ periods. There are one risk-free asset and $d$ risky assets in the financial market. Moreover, we assume that a sequence of $\mathbb{R}^{K}$-valued random vectors, denoted by $\left(F_{t}\right)_{t=0}^{T}$, influences distributions of the return vectors of the risky assets: The return vector of the risky assets at time $t$, denoted by $R_{t}$, satisfies

$$
R_{t}=\mu\left(F_{t}\right)+\sigma\left(F_{t}\right) \epsilon_{t}, \quad t \geq 0,
$$

where $\left(\epsilon_{t}\right)_{t=1}^{T}$ is a sequence of mutually independent and $d$-dimensional standard normal random vectors, and $\mu: \mathbb{R}^{K} \rightarrow \mathbb{R}^{d}$ and $\sigma: \mathbb{R}^{K} \rightarrow \mathbb{R}^{d \times d}$ are measurable functions. Furthermore, 
we assume that $F_{t}$ and $\epsilon_{t}$ are mutually independent for each $t=1, \ldots, T$. Then, the conditional expectation and variance of $R_{t}$ given by $F_{t}$ are

$$
\begin{aligned}
\mathrm{E}\left[R_{t} \mid F_{t}\right] & =\mu\left(F_{t}\right), \\
\operatorname{Var}\left(R_{t} \mid F_{t}\right) & =\sigma\left(F_{t}\right)\left(\sigma\left(F_{t}\right)\right)^{\prime},
\end{aligned}
$$

for all $t \geq 1$. Hence, the conditional mean and variance of the risky assets' returns are driven by the movement of $\left(F_{t}\right)_{t=0}^{T}$. For convenience, we write $R_{0}=\mathbf{0}_{d}$. The risk-free rate is a constant over time and it is denoted by $r_{\mathrm{f}}$, and $\left(R_{t}^{e}\right)_{t=1}^{T}$ is the excess return process, $R_{t}^{e}=R_{t}-r_{\mathrm{f}} \mathbf{1}_{d}$.

Denote by $\mathbb{F}^{R, F}=\left(\mathcal{F}_{t}^{R, F}\right)_{t=0}^{T}$ and $\mathbb{F}^{F}=\left(\mathcal{F}_{t}^{F}\right)_{t=0}^{T}$ the filtrations generated by $\left(R_{t}, F_{t}\right)_{t=0}^{T}$ and $\left(F_{t}\right)_{t=0}^{T}$, respectively. We assume that an investor can observe the value of $F_{t}$ and $R_{t}$ at each $t \geq 0$, so her information at time $t$ is represented by $\mathcal{F}_{t}^{R, F}$. In addition, we assume the following:

Assumption 1 The random process $\left(F_{t}\right)_{t=0}^{T}$ is a time-homogeneous Markov process with respect to $\mathbb{F}^{R, F}$.

Assumption 1 is important for our optimization. Many return models satisfy this assumption.

\section{Example 2}

1. Factor pricing models. Suppose that $\left(F_{t}\right)_{t=0}^{T}$ satisfies Assumption 1. Let $\mu(F)=r_{\mathrm{f}} \mathbf{1}_{d}+$ $B F$ and $\sigma(F)=\bar{\sigma}$, where $B \in \mathbb{R}^{d, K}$ and $\bar{\sigma} \in \mathbb{R}^{d, d}$ are constant matrices. Then, the return process can be expressed as

$$
R_{t}=r_{\mathrm{f}} \mathbf{1}_{d}+B F_{t}+\bar{\sigma} \epsilon_{t}, \quad t \geq 1 .
$$

The above return process is the one of factor pricing models such as the capital asset pricing model (CAPM) and the Fama-French three factor model.

2. Stochastic volatility models. Suppose that $K=d$ and that $\left(F_{t}\right)_{t=0}^{T}$ is specified by

$$
\log \left(F_{t}\right)=m+B\left(\log \left(F_{t-1}\right)-m\right)+\eta_{t}, \quad t \geq 1,
$$

where $m \in \mathbb{R}^{d}$ is a constant vector and $B \in \mathbb{R}^{d, d}$ is a constant diagonal matrix. $\left(\eta_{t}\right)_{t=1}^{T}$ is a sequence of $d$-dimensional i.i.d. random vectors that are independent of $\left(\epsilon_{t}\right)_{t=1}^{T}$. The return process is defined as

$$
R_{t}=\bar{\mu}+\operatorname{diag}\left(\sqrt{F_{t}}\right) C \epsilon_{t}, \quad t \geq 1
$$

where $C C^{\prime}$ is a constant correlation matrix. Then, $\left(F_{t}\right)_{t=0}^{T}$ satisfies Assumption 1.

3. Markov regime-switching models. Suppose that $\left(F_{t}\right)_{t=0}^{T}$ is a $K$-states, time-homogeneous Markov chain and that $\mu$ and $\sigma$ are specified by

$$
\mu\left(F_{t}\right)=\mu_{i}, \quad \sigma\left(F_{t}\right)=\sigma_{i}, \quad \text { if } F_{t} \text { is } i \text { th state. }
$$

In addition, $\left(F_{t}\right)_{t=0}^{T}$ and $\left(\epsilon_{t}\right)_{t=1}^{T}$ are mutually independent. Then, $\left(F_{t}\right)_{t=0}^{T}$ satisfies Assumption 1. 
In usual portfolio optimization, researchers assume that an investor knows the conditional mean $\mu\left(F_{t}\right)$ and variance $\sigma\left(F_{t}\right)\left(\sigma\left(F_{t}\right)\right)^{\prime}$, but, the investor needs to estimate the moments in practice. In the robust optimization literature, researchers take into account the estimation error of these moments. Similar to the literature, we introduce two statistical error components. Let $\left(\theta_{t}\right)_{t=0}^{T-1}$ be a $\mathbb{R}^{d}$-valued random process of errors of mean and let $\left(V_{t}\right)_{t=0}^{T-1}$ be a $\mathbb{R}^{d, d}$-valued process of errors of variance. The one-period-ahead conditional mean and variance with respect to the probability measure representing the investor's belief, denoted by $\mathbb{P}^{\theta, V}$, can be expressed as

$\mathrm{E}^{\theta, V}\left[R_{t+1} \mid \mathcal{F}_{t}^{R, F}\right]=\mathrm{E}\left[R_{t+1} \mid \mathcal{F}_{t}^{R, F}\right]+\theta_{t}, \quad \operatorname{Var}^{\theta, V}\left(R_{t+1} \mid \mathcal{F}_{t}^{R, F}\right)=\operatorname{Var}\left(R_{t+1} \mid \mathcal{F}_{t}^{R, F}\right)+V_{t}, \quad t \geq 0$.

Hence, $\theta_{t}$ and $V_{t}$ can be regarded as statistical errors of conditional mean and variance given $\mathcal{F}_{t}^{R, F}$ at each $t$. The assumptions regarding $\left(\theta_{t}\right)_{t=0}^{T-1}$ and $\left(V_{t}\right)_{t=0}^{T-1}$ are

\section{Assumption 3}

1. For each $t, \theta_{t}$ and $V_{t}$ are $\mathcal{F}_{t}^{R, F}$-measurable.

2. For each $t, \operatorname{Var}\left(R_{t+1} \mid \mathcal{F}_{t}^{R, F}\right)+V_{t}$ and $\sigma\left(F_{t+1}\right)\left(\sigma\left(F_{t+1}\right)\right)^{\prime}+V_{t}$ are positive-definite matrices. From the definitions of $\left(\theta_{t}\right)_{t=0}^{T-1}$ and $\left(V_{t}\right)_{t=0}^{T-1}$, Assumption 3 is natural.

The important technical issue is whether the probability measure $\mathbb{P}^{\theta, V}$ exists or not. To construct the probability measure $\mathbb{P}^{\theta, V}$, we introduce the random variable,

$$
Z_{t}=\prod_{s=0}^{t} \zeta_{s}, \quad t \geq 0
$$

where

$$
\begin{aligned}
& \zeta_{t+1}= \sqrt{\frac{\operatorname{det}\left(\sigma\left(F_{t+1}\right)\left(\sigma\left(F_{t+1}\right)\right)^{\prime}\right)}{\operatorname{det}\left(\sigma\left(F_{t+1}\right)\left(\sigma\left(F_{t+1}\right)\right)^{\prime}+V_{t}\right)}} \\
& \times \exp \left\{-\frac{1}{2} \theta_{t}^{\prime}\left(\sigma\left(F_{t+1}\right)\left(\sigma\left(F_{t+1}\right)\right)^{\prime}+V_{t}\right)^{-1} \theta_{t}+\theta_{t}^{\prime}\left(\sigma\left(F_{t+1}\right)\left(\sigma\left(F_{t+1}\right)\right)^{\prime}+V_{t}\right)^{-1} \sigma\left(F_{t+1}\right) \epsilon_{t+1}\right. \\
&\left.\quad+\frac{1}{2} \epsilon_{t+1}^{\prime}\left(I_{d}-\left(\sigma\left(F_{t+1}\right)\right)^{\prime}\left(\sigma\left(F_{t+1}\right)\left(\sigma\left(F_{t+1}\right)\right)^{\prime}+V_{t}\right)^{-1} \sigma\left(F_{t+1}\right)\right) \epsilon_{t+1}\right\}, \quad t \geq 1
\end{aligned}
$$

and $\zeta_{0}=1$. Then, the following lemma holds.

Lemma 4 Under Assumptions 1 and 3, there exists a probability measure $\mathbb{P}^{\theta, V}$, such that

$$
\mathbb{P}^{\theta, V}(A)=\mathrm{E}\left[\mathbb{1}_{A} Z_{T}\right], \quad A \in \mathcal{F}_{T}^{R, F} .
$$

Under $\mathbb{P}^{\theta, V}$,

$$
\begin{aligned}
\mathrm{E}^{\theta, V}\left[R_{t+1} \mid \mathcal{F}_{t}^{R, F}\right] & =\mathrm{E}\left[R_{t+1} \mid \mathcal{F}_{t}^{R, F}\right]+\theta_{t}, \\
\operatorname{Var}^{\theta, V}\left(R_{t+1} \mid \mathcal{F}_{t}^{R, F}\right) & =\operatorname{Var}\left(R_{t+1} \mid \mathcal{F}_{t}^{R, F}\right)+V_{t},
\end{aligned}
$$

for all $t \geq 0$. Furthermore, the conditional distribution of $F_{t+1}$ given $\mathcal{F}_{t}^{R, F}$ under $\mathbb{P}^{\theta, V}$ is the same as the conditional distribution of $F_{t+1}$ given $\mathcal{F}_{t}^{R, F}$ under $\mathbb{P}$. 
The proof of Lemma 4 is in Section A.1. Since $\left(F_{t}\right)_{t=0}^{T}$ is the time-homogeneous Markov process with respect to $\mathbb{F}^{R, F}$, we can express the conditional mean and variance of $R_{t+1}$ as functions depending on $F_{t}$, that is,

$$
\begin{aligned}
& m(F):=\mathrm{E}\left[R_{t+1} \mid F_{t}=F\right]=\mathrm{E}\left[R_{t+1} \mid \mathcal{F}_{t}^{R, F}\right], \\
& A(F):=\operatorname{Var}\left(R_{t+1} \mid F_{t}=F\right)=\operatorname{Var}\left(R_{t+1} \mid \mathcal{F}_{t}^{R, F}\right),
\end{aligned}
$$

for all $t \geq 0$. We denote the conditional expected excess return by $m^{e}(F)=m(F)-r_{\mathrm{f}} \mathbf{1}_{d}$.

Now, let us formulate the optimization problem of the investor. Let $\left(X_{t}\right)_{t=0}^{T}$ be a wealth process of the investor. We assume that $X_{t}$ satisfies a self-financing constraint, that is,

$$
X_{t+1}=\left(\left(1+r_{\mathrm{f}}\right)+\left(R_{t+1}^{e}\right)^{\prime} u_{t}\right) X_{t}, \quad t \geq 0
$$

where $\left(u_{t}\right)_{t=0}^{T-1}$ is a sequence of $d$-dimensional portfolio vectors for risky assets. For each $u_{t}$, the $i$ th element of $u_{t}$ represents the weight of the wealth invested in the $i$ th risky asset. The investor buys or sells $X_{t}\left(1-\left(\mathbf{1}_{d}\right)^{\prime} u_{t}\right)$ dollar's risk-free asset at each $t \geq 0$. The investor's objective function is

$$
\mathrm{E}^{\theta, V}\left[\sum_{t=0}^{T-1} \delta^{t}\left(\mathrm{E}^{\theta, V}\left[X_{t+1} \mid \mathcal{F}_{t}^{R, F}\right]-\frac{\widehat{\gamma}_{t}}{2 X_{t}} \operatorname{Var}^{\theta, V}\left(X_{t+1} \mid \mathcal{F}_{t}^{R, F}\right)\right)\right],
$$

where $\widehat{\gamma}:=\left(\widehat{\gamma}_{t}\right)_{t=0}^{T-1}$ is a positive and $\mathbb{F}^{F}$-adapted process which represents the investor's trade-off between the expected return and associated risks. We emphasize that $\widehat{\gamma}$ is not only $\mathbb{F}^{R, F}$-adapted, but also $\mathbb{F}^{F}$-adapted. The $\mathbb{F}^{F}$-adaptedness of $\widehat{\gamma}$ is important in the derivation of the explicit form of the value function.

The trade-off parameter is divided by $X_{t}$ whereas in the other standard problem, it is not divided. This parameterization is used by Björk, Murgoci, and Zhou (2014). They provide two natural interpretations of the parameterization. One is an adjustment of units. The unit of the conditional expected wealth is (dollar), whereas the unit of the conditional variance is $(\text { dollar })^{2}$. So the variance needs to be divided by wealth in order to measure the objective function in dollars. Another interpretation is to measure the objective function by the rate of return. We replace the wealth in the objective by the gross return of the wealth, namely, we consider

$$
\begin{aligned}
& \mathrm{E}^{\theta, V}\left[\frac{X_{t+1}}{X_{t}} \mid \mathcal{F}_{t}^{R, F}\right]-\frac{\widehat{\gamma}_{t}}{2} \operatorname{Var}^{\theta, V}\left(\frac{X_{t+1}}{X_{t}} \mid \mathcal{F}_{t}^{R, F}\right) \\
& =\frac{1}{X_{t}}\left\{\mathrm{E}^{\theta, V}\left[X_{t+1} \mid \mathcal{F}_{t}^{R, F}\right]-\frac{\widehat{\gamma}_{t}}{2 X_{t}} \operatorname{Var}^{\theta, V}\left(X_{t+1} \mid \mathcal{F}_{t}^{R, F}\right)\right\} .
\end{aligned}
$$

Then, Björk et al. (2014) argue that the equilibrium in the above objective is the same as that in the following objective,

$$
\mathrm{E}^{\theta, V}\left[X_{t+1} \mid \mathcal{F}_{t}^{R, F}\right]-\frac{\widehat{\gamma}_{t}}{2 X_{t}} \operatorname{Var}^{\theta, V}\left(X_{t+1} \mid \mathcal{F}_{t}^{R, F}\right) .
$$

However, in our settings, the optimal portfolios are different between these two objectives. Our settings, therefore, should be interpreted as the adjustment of units.

We can also interpret our objective function as an approximation of a certainty equivalent of a CRRA utility. Let

$$
u_{t}(x):= \begin{cases}\frac{x^{1-\widehat{\gamma}_{t}}-1}{1-\widehat{\gamma}_{t}}, & \text { if } \widehat{\gamma}_{t} \neq 1 \\ \log x, & \text { if } \widehat{\gamma}_{t}=1\end{cases}
$$


where $\widehat{\gamma}_{t}>0$ is the constant relative risk aversion coefficient at time $t$. Let us denote by $\bar{c}_{t}(X)$ the certainty equivalent of a random variable $X$ under the probability measure $\mathbb{P}^{\theta, V}$, that is

$$
\bar{c}_{t}(X):=\left(u_{t}\right)^{-1}\left(\mathrm{E}^{\theta, V}\left[u_{t}(X) \mid \mathcal{F}_{t}^{R, F}\right]\right),
$$

where $\left(u_{t}\right)^{-1}$ is the functional inverse of $u_{t}$. Then, it is well known that $\bar{c}_{t}(X)$ can be approximated as follows.

$$
\bar{c}_{t}\left(X_{t+1}\right) \approx \mathrm{E}^{\theta, V}\left[X_{t+1} \mid \mathcal{F}_{t}^{R, F}\right]+\frac{1}{2} \frac{u_{t}^{\prime \prime}\left(\mathrm{E}^{\theta, V}\left[X_{t+1} \mid \mathcal{F}_{t}^{R, F}\right]\right)}{u_{t}^{\prime}\left(\mathrm{E}^{\theta, V}\left[X_{t+1} \mid \mathcal{F}_{t}^{R, F}\right]\right)} \operatorname{Var}^{\theta, V}\left(X_{t+1} \mid \mathcal{F}_{t}^{R, F}\right),
$$

where $u_{t}^{\prime}$ and $u_{t}^{\prime \prime}$ are the first and the second derivatives of $u_{t}$, respectively. Now, we assume $X_{t} \approx \mathrm{E}^{\theta, V}\left[X_{t+1} \mid \mathcal{F}_{t}^{R, F}\right]$. Then, since $u_{t}$ is a CRRA utility, we have

$$
\frac{u_{t}^{\prime \prime}\left(\mathrm{E}^{\theta, V}\left[X_{t+1} \mid \mathcal{F}_{t}^{R, F}\right]\right)}{u_{t}^{\prime}\left(\mathrm{E}^{\theta, V}\left[X_{t+1} \mid \mathcal{F}_{t}^{R, F}\right]\right)}=-\frac{\widehat{\gamma}_{t}}{\mathrm{E}^{\theta, V}\left[X_{t+1} \mid \mathcal{F}_{t}^{R, F}\right]} \approx-\frac{\widehat{\gamma}_{t}}{X_{t}}
$$

Therefore, we have

$$
\bar{c}_{t}\left(X_{t+1}\right) \approx \mathrm{E}^{\theta, V}\left[X_{t+1} \mid \mathcal{F}_{t}^{R, F}\right]-\frac{\widehat{\gamma}_{t}}{2 X_{t}} \operatorname{Var}^{\theta, V}\left(X_{t+1} \mid \mathcal{F}_{t}^{R, F}\right) .
$$

Hence, the expectation of the discounted sum of the certainty equivalents can be approximated as follows:

$$
\mathrm{E}^{\theta, V}\left[\sum_{t=0}^{T-1} \delta^{t} \bar{c}_{t}\left(X_{t+1}\right)\right] \approx \mathrm{E}^{\theta, V}\left[\sum_{t=0}^{T-1} \delta^{t}\left(\mathrm{E}^{\theta, V}\left[X_{t+1} \mid \mathcal{F}_{t}^{R, F}\right]-\frac{\widehat{\gamma}_{t}}{2 X_{t}} \operatorname{Var}^{\theta, V}\left(X_{t+1} \mid \mathcal{F}_{t}^{R, F}\right)\right)\right] .
$$

The above approximation coincides with our objective function (2.1). Furthermore, the time varying $\widehat{\gamma}$ implies that the relative risk aversion coefficient varies over time.

Note that, we assume that $X_{t}$ is almost surely strictly positive for all $t \geq 0$. Strictly speaking, it does not hold when permitting short selling, whereas it holds if short selling is not allowed. Nevertheless, we assume the strict positivity of $X_{t}$ in all cases for mathematical convenience. Furthermore, the division of the trade-off parameter by $X_{t}$ is a crucial assumption for the derivation of the explicit solution to our problem. Without it, we can not derive the explicit solution. We again refer to this assumption in the problem without the risk-free asset.

By the Markov property of $\left(F_{t}\right)_{t=0}^{T}$, the objective under $\mathbb{P}^{\theta, V}$ can be expressed as

$$
\begin{aligned}
& \mathrm{E}^{\theta, V}\left[\sum_{t=0}^{T-1} \delta^{t}\left(\mathrm{E}^{\theta, V}\left[X_{t+1} \mid \mathcal{F}_{t}^{R, F}\right]-\frac{\widehat{\gamma}_{t}}{2 X_{t}} \operatorname{Var}^{\theta, V}\left(X_{t+1} \mid \mathcal{F}_{t}^{R, F}\right)\right)\right] \\
& =\mathrm{E}^{\theta, V}\left[\sum_{t=0}^{T-1} \delta^{t} X_{t} c_{t}\left(F_{t}, \theta_{t}, V_{t}, u_{t}\right)\right],
\end{aligned}
$$

where $c_{t}: \mathbb{R}^{K} \times \mathbb{R}^{d} \times \mathbb{R}^{d, d} \times \mathbb{R}^{d} \rightarrow \mathbb{R}$ is a measurable function such that

$$
c_{t}(F, \theta, V, u)=1+r_{\mathrm{f}}+\left(m^{e}(F)+\theta\right)^{\prime} u-\frac{\widehat{\gamma}_{t}}{2} u^{\prime}(A(F)+V) u .
$$

Now, we construct the optimization problem with multiple priors. The investor selects the portfolio maximizing the expected utility of the worst case. This means that the investor 
faces on the following maximization problem:

$$
\begin{gathered}
V_{T}(x, f)=\max _{\substack{\left.\left(u_{t}\right)_{t=0}^{T-1} \in \mathcal{A}_{T}^{u} \\
\text { subject to } X_{t+1}, V_{t}\right)_{t=0}^{T-1} \in \mathcal{A}_{T}^{\theta, V}}} \mathrm{E}^{\theta, V}\left[\sum_{t=0}^{T-1} \delta^{t} X_{t} c_{t}\left(F_{t}, \theta_{t}, V_{t}, u_{t}\right)\right] \\
X_{0}=x, \quad R_{0}=f,
\end{gathered}
$$

where $\mathcal{A}_{T}^{u}$ is a set of admissible portfolios and $\mathcal{A}_{T}^{\theta, V}$ is a set of admissible errors, $\left(\theta_{t}\right)_{t=0}^{T-1}$ and $\left(V_{t}\right)_{t=0}^{T-1}$. The set of admissible portfolios can be expressed as

$$
\mathcal{A}_{T}^{u}=\left\{u=\left(u_{t}\right)_{t=0}^{T-1} \mid u \text { is a } \mathbb{R}^{d} \text {-valued and } \mathbb{F}^{R, F} \text {-adapted process. }\right\} .
$$

So, the investor is permitted to short sell. We later analyze the case when the investor is prohibited from short selling.

The set of admissible errors is characterized by two inequalities. For all $\left(\theta_{t}, V_{t}\right)_{t=0}^{T-1} \in \mathcal{A}_{T}^{\theta, V}$, $\left(\theta_{t}\right)_{t=0}^{T-1}$ is a $\mathbb{R}^{d}$-valued process and $\left(V_{t}\right)_{t=0}^{T-1}$ is an $\mathbb{R}^{d \times d}$-valued process. Furthermore, they satisfy Assumption 3 and

$$
\begin{aligned}
& \left(\eta_{t}^{\theta}\right)^{2} \geq \theta_{t}^{\prime}\left(A\left(F_{t}\right)+V_{t}\right)^{-1} \theta_{t}, \\
& \left(\eta_{t}^{V}\right)^{2} \operatorname{tr}\left(\left(A\left(F_{t}\right)\right)^{2}\right) \geq \operatorname{tr}\left(V_{t}^{2}\right),
\end{aligned}
$$

where $\left(\eta_{t}^{\theta}\right)_{t=0}^{T-1}$ and $\left(\eta_{t}^{V}\right)_{t=0}^{T-1}$ are positive $\mathbb{F}^{F}$-adopted processes and $\operatorname{tr}(A)$ is the trace of matrix $A$.

The inequality (2.3) represents a confidence interval in statistics. This idea is introduced by Garlappi et al. (2007). We can express $\theta_{t}^{\prime}\left(A\left(F_{t}\right)+V_{t}\right)^{-1} \theta_{t}$ as

$$
\begin{aligned}
& \theta_{t}^{\prime}\left(\operatorname{Var}^{\theta, V}\left(R_{t+1} \mid \mathcal{F}_{t}^{R, F}\right)\right)^{-1} \theta_{t}= \\
& \left(\mathrm{E}\left[R_{t+1} \mid \mathcal{F}_{t}^{R, F}\right]-\mathrm{E}^{\theta, V}\left[R_{t+1} \mid \mathcal{F}_{t}^{R, F}\right]\right)^{\prime}\left(\operatorname{Var}^{\theta, V}\left(R_{t+1} \mid \mathcal{F}_{t}^{R, F}\right)\right)^{-1}\left(\mathrm{E}\left[R_{t+1} \mid \mathcal{F}_{t}^{R, F}\right]-\mathrm{E}^{\theta, V}\left[R_{t+1} \mid \mathcal{F}_{t}^{R, F}\right]\right),
\end{aligned}
$$

so $\theta_{t}^{\prime}\left(A\left(F_{t}\right)+V_{t}\right)^{-1} \theta_{t}$ is the $F$ statistic of the estimated means in the null hypothesis of $\mathrm{E}\left[R_{t+1} \mid \mathcal{F}_{t}^{R, F}\right]=\mathrm{E}^{\theta, V}\left[R_{t+1} \mid \mathcal{F}_{t}^{R, F}\right]$.

Garlappi et al. (2007) characterize this constraint of $\left(\theta_{t}\right)_{t=0}^{T-1}$ as the confidence interval of the estimated mean vector. Their discussion is justified if the return vectors of the risky assets are i.i.d.. However, the return vectors in our model are not i.i.d. in general. Therefore, our constraint (2.3) does not represent the confidence interval. Since the financial models are usually estimated by using the maximum likelihood methods, one should use the inverse of the information matrix as the covariance matrix in our constraint (2.3) in order to characterize this constraint as the confidence interval. However, in this paper, we use this constraint, similar to Garlappi et al. (2007), to reduce the mathematical complexity.

The inequality (2.4) can be expressed as

$$
\eta_{t}^{V} \geq \frac{\operatorname{tr}\left(V_{t}^{2}\right)}{\operatorname{tr}\left(\left(A\left(F_{t}\right)\right)^{2}\right)}=\frac{\left\|\operatorname{Var}^{\theta, V}\left(R_{t+1} \mid \mathcal{F}_{t}^{R, F}\right)-\operatorname{Var}\left(R_{t+1} \mid \mathcal{F}_{t}^{R, F}\right)\right\|^{2}}{\left\|\operatorname{Var}\left(R_{t+1} \mid \mathcal{F}_{t}^{R, F}\right)\right\|^{2}},
$$

for all $t \geq 0$. Therefore, $\left(\eta_{t}^{V}\right)_{t=0}^{T-1}$ represents the upper boundary of the ratio of the least square error to the conditional variance. Ledoit and Wolf (2003) consider the shrinkage estimator minimizing the following objective:

$$
\min _{\alpha} \operatorname{tr}\left((\widehat{\Sigma}(\alpha)-\Sigma)^{2}\right)=\min _{\alpha}\|\widehat{\Sigma}(\alpha)-\Sigma\|^{2},
$$


where $\Sigma$ is an actual covariance matrix of returns and $\widehat{\Sigma}(\alpha)$ is a shrunk estimated covariance matrix depending on the parameter $\alpha$. We use the above objective in the inequality (2.4). Therefore, the inequality (2.4) represents the upper boundary of the objective in Ledoit and Wolf (2003).

As in the case of a unique prior, we can apply the dynamic programming procedure to the optimization problem (2.2).

Theorem 5 Let $\left(Y_{t}^{T}\right)_{t=0}^{T-1}$ be a sequence of random variables, such that

$$
\begin{aligned}
Y_{T-1}^{T} & =\max _{u_{T-1} \in \mathbb{R}^{d}} \min _{\left.\left(\theta_{T-1}, V_{T-1}\right) \in \mathcal{A}_{T}^{\theta, V}\right|_{T-1} ^{T-1}}\left\{c_{T-1}\left(F_{T-1}, \theta_{T-1}, V_{T-1}, u_{T-1}\right)\right\}, \\
Y_{t}^{T} & =\max _{u_{t} \in \mathbb{R}^{d}} \min _{\left.\left(\theta_{t}, V_{t}\right) \mathcal{A}_{T}^{\theta, V}\right|_{t} ^{t}}\left\{c_{t}\left(F_{t}, \theta_{t}, V_{t}, u_{t}\right)+\delta \mathrm{E}^{\theta, V}\left[Y_{t+1}^{T}\left(1+r_{\mathrm{f}}+\left(R_{t+1}^{e}\right)^{\prime} u_{t}\right) \mid \mathcal{F}_{t}^{R, S}\right]\right\},
\end{aligned}
$$

for all $0 \leq t \leq T-2$, where $\left.\mathcal{A}_{T}^{\theta, V}\right|_{t} ^{t}$ is a set of $t$-th elements in $\mathcal{A}_{T}^{\theta, V}$. Then, the above random sequence exists, and

$$
V_{T}(x, f)=\mathrm{E}\left[Y_{0}^{T} \mid F_{0}=f\right] x, \quad x \in \mathbb{R}, f \in \mathbb{R}^{K} .
$$

Furthermore, the optimal portfolio process $\left(u_{t}^{*}\right)_{t=0}^{T-1}$ derived by the above dynamic programming is time-consistent.

The explicit solution is provided by the following proposition.

\section{Proposition 6 Let}

$$
\begin{aligned}
Y_{T}^{T} & =0 \\
m_{t}^{e} & =\mathrm{E}\left[\left(1+\delta Y_{t+1}^{T}\right)\left(\mu\left(F_{t+1}\right)-r_{\mathrm{f}} \mathbf{1}_{d}\right) \mid \mathcal{F}_{t}^{R, F}\right], \quad g_{t}^{e}=1+\delta \mathrm{E}\left[Y_{t+1}^{T} \mid \mathcal{F}_{t}^{R, F}\right], \\
a_{t}^{e} & =\left(m_{t}^{e}\right)^{\prime}\left(A\left(F_{t}\right)+\eta_{t}^{V}\left\|A\left(F_{t}\right)\right\| I_{d}\right)^{-1} m_{t}^{e}
\end{aligned}
$$

for all $0 \leq t \leq T-1$. Then, $\left(Y_{t}^{T}\right)_{t=0}^{T-1}$ in Theorem 5 can be expressed as

$$
Y_{t}^{T}=\left(1+r_{\mathrm{f}}\right) g_{t}^{e}+\frac{\left(\left(\sqrt{a_{t}^{e}}-\left|g_{t}^{e}\right| \eta_{t}^{\theta}\right)^{+}\right)^{2}}{2 \widehat{\gamma}_{t}}
$$

and the optimal portfolio is

$$
u_{t}^{*}=\frac{1}{\widehat{\gamma}_{t}}\left(1-\frac{\left|g_{t}^{e}\right| \eta_{t}^{\theta}}{\sqrt{a_{t}^{e}}}\right)^{+}\left(A\left(F_{t}\right)+\eta_{t}^{V}\left\|A\left(F_{t}\right)\right\| I_{d}\right)^{-1} m_{t}^{e} .
$$

The proofs of Theorem 5 and Proposition 6 are in Section A.2. By the construction, $\left(Y_{t}^{T}\right)_{t=0}^{T-1}$ is $\mathbb{F}^{F}$-adapted. Furthermore, if $\widehat{\gamma}_{t}, \eta_{t}^{\theta}$ and $\eta_{t}^{V}$ depend only on the value of $F_{t}$ for all $0 \leq t \leq T-1, Y_{t}^{T}$ also depends only on the value of $F_{t}$. Therefore, we can easily compute it numerically using the Markov property of $\left(F_{t}\right)_{t=0}^{T}$.

The optimal portfolio (2.5) is similar to the ambiguity averse minimum-variance portfolio by Pinar (2014). However, the multiple priors for mean and variance affects the portfolio (2.5) whereas the ambiguity averse minimum-variance portfolio is only affected by the multiple priors for mean. Therefore, the ambiguity averse minimum-variance portfolio is a special case of our portfolios.

Note that, we obtain similar results to Proposition 6 even if $r_{\mathrm{f}}$ varies over time. Let $r_{\mathrm{f}, t+1}$ be a time-varying risk-free rate at time $t+1$ such that

$$
r_{\mathrm{f}, t+1}=h\left(F_{t}\right), \quad t \geq 0,
$$


where $h$ is a measurable function from $\mathbb{R}^{K}$ onto $\mathbb{R}$. The reason why the risk-free rate at time $t+1$ depends on the value of $F_{t}$ is that the risk-free rate needs to be determined before investment decision. Then, the following portfolio $u_{t}^{T V *}$ is optimal.

$$
u_{t}^{T V *}=\frac{1}{\widehat{\gamma}_{t}}\left(1-\frac{\left|g_{t}^{T V e}\right| \eta_{t}^{\theta}}{\sqrt{a_{t}^{T V e}}}\right)^{+}\left(A\left(F_{t}\right)+\eta_{t}^{V}\left\|A\left(F_{t}\right)\right\| I_{d}\right)^{-1} m_{t}^{T V e},
$$

where

$$
\begin{aligned}
Y_{T}^{T V T} & =0, \quad m_{t}^{T V e}=\mathrm{E}\left[\left(1+\delta Y_{t+1}^{T V T}\right)\left(\mu\left(F_{t+1}\right)-h\left(F_{t}\right) \mathbf{1}_{d}\right) \mid \mathcal{F}_{t}^{R, F}\right], \\
g_{t}^{T V e} & =1+\delta \mathrm{E}\left[Y_{t+1}^{T V T} \mid \mathcal{F}_{t}^{R, F}\right], \quad a_{t}^{T V e}=\left(m_{t}^{T V e}\right)^{\prime}\left(A\left(F_{t}\right)+\eta_{t}^{V}\left\|A\left(F_{t}\right)\right\| I_{d}\right)^{-1} m_{t}^{T V e}, \\
Y_{t}^{T V T} & =\left(1+h\left(F_{t}\right)\right) g_{t}^{T V e}+\frac{\left(\left(\sqrt{a_{t}^{T V e}}-\left|g_{t}^{T V e}\right| \eta_{t}^{\theta}\right)^{+}\right)^{2}}{2 \widehat{\gamma}_{t}} .
\end{aligned}
$$

It is clear that $Y_{t}^{T V T}$ is $\mathcal{F}_{t}^{R, F}$-measurable for all $t \geq 0$. In addition, $u^{T V *}$ is admissible. The proof is similar to that of Proposition 6, so we omit it. The above result indicates that if the movement of $r_{\mathrm{f}, t}$ is independent of $\epsilon_{t}$, then the optimal portfolio with the constant risk-free rate can be naturally extended into the optimal portfolio with the time-varying risk-free rate.

We next consider an optimization problem with multiple priors without the risk-free asset. In this case, the investor's wealth updating formula is

$$
X_{t+1}=\left(1+\left(R_{t+1}\right)^{\prime} u_{t}\right) X_{t}, \quad 0 \leq t \leq T-1,
$$

where $u_{t}$ satisfies $\mathbf{1}_{d}^{\prime} u_{t}=1$. The objective can be expressed as

$$
\begin{aligned}
& \mathrm{E}^{\theta, V}\left[\sum_{t=0}^{T-1} \delta^{t}\left(\mathrm{E}^{\theta, V}\left[X_{t+1} \mid \mathcal{F}_{t}^{R, F}\right]-\frac{\widehat{\gamma}_{t}}{2 X_{t}} \operatorname{Var}^{\theta, V}\left(X_{t+1} \mid \mathcal{F}_{t}^{R, F}\right)\right)\right] \\
& =\mathrm{E}^{\theta, V}\left[\sum_{t=0}^{T-1} \delta^{t} X_{t} \widehat{c}_{t}\left(F_{t}, \theta_{t}, V_{t}, u_{t}\right)\right],
\end{aligned}
$$

where $\widehat{c}_{t}: \mathbb{R}^{K} \times \mathbb{R}^{d} \times \mathbb{R}^{d, d} \times \mathbb{R}^{d} \rightarrow \mathbb{R}$ is a measurable function such that

$$
\widehat{c}_{t}(F, \theta, V, u)=1+(m(F)+\theta)^{\prime} u-\frac{\widehat{\gamma}_{t}}{2} u^{\prime}(A(F)+V) u .
$$

Hence, the objective without the risk-free asset has the same form as the objective with the risk-free asset. This implies that Theorem 5 can be applied to the problem without the risk-free asset.

The optimization problem without the risk-free asset is formulated as

$$
\begin{gathered}
V_{T}^{\text {worf }}(x, f)=\max _{\left(u_{t}\right)_{t=0}^{T-1} \in \widetilde{\mathcal{A}}_{T}^{u}} \min _{\left(\theta_{t}, V_{t}\right)_{t=0}^{T-1} \in \mathcal{A}_{T}^{\theta, V}} \mathrm{E}^{\theta, V}\left[\sum_{t=0}^{T-1} \delta^{t} X_{t} \widehat{c}_{t}\left(F_{t}, \theta_{t}, V_{t}, u_{t}\right)\right] \\
\text { subject to } X_{t+1}=\left(1+\left(R_{t+1}\right)^{\prime} u_{t}\right) X_{t}, \quad t \geq 0, \\
X_{0}=x, \quad F_{0}=f,
\end{gathered}
$$

where the set of admissible portfolios $\widetilde{\mathcal{A}}_{T}^{u}$ is

$$
\widetilde{\mathcal{A}}_{T}^{u}=\left\{u=\left(u_{t}\right)_{t=0}^{T-1} \mid u \text { is a } \mathbb{R}^{d} \text {-valued and } \mathbb{F}^{R, F} \text {-adapted process and } \mathbf{1}_{d}^{\prime} u_{t}=1\right\} .
$$

The following proposition provides the optimal portfolios and the value functions. 
Proposition 7 For all $0 \leq t \leq T-1$, let

$$
\begin{aligned}
\widehat{Y}_{T}^{T} & =0 \\
g_{t} & =1+\delta \mathrm{E}\left[\widehat{Y}_{t+1}^{T} \mid \mathcal{F}_{t}^{R, F}\right], \quad m_{t}=\mathrm{E}\left[\left(1+\delta \widehat{Y}_{t+1}^{T}\right) \mu\left(F_{t+1}\right) \mid \mathcal{F}_{t}^{R, F}\right], \\
a_{t}^{\text {worf }} & =m_{t}^{\prime}\left(A\left(F_{t}\right)+\eta_{t}^{V}\left\|A\left(F_{t}\right)\right\| I_{d}\right)^{-1} m_{t}, \quad c_{t}^{\text {worf }}=m_{t}^{\prime}\left(A\left(F_{t}\right)+\eta_{t}^{V}\left\|A\left(F_{t}\right)\right\| I_{d}\right)^{-1} \mathbf{1}_{d}, \\
b_{t}^{\text {worf }} & =\mathbf{1}_{d}^{\prime}\left(A\left(F_{t}\right)+\eta_{t}^{V}\left\|A\left(F_{t}\right)\right\| I_{d}\right)^{-1} \mathbf{1}_{d}, \quad d_{t}^{\text {worf }}=a_{t}^{\text {worf }} b_{t}^{\text {worf }}-\left(c_{t}^{\text {worf }}\right)^{2}, \\
\widehat{Y}_{t}^{T} & =g_{t}+\frac{c_{t}^{\text {worf }}-\left(\widehat{\gamma}_{t}+\left|g_{t}\right| \eta_{t}^{\theta} / \psi_{t}^{*}\right)}{b_{t}^{\text {worf }}}+\frac{\widehat{\gamma}_{t}}{2}\left(\psi_{t}^{*}\right)^{2},
\end{aligned}
$$

where $\psi_{t}^{*}$ is a unique positive solution of the polynomial equation.

$$
b_{t}^{\text {worf }}\left(\psi_{t}^{*}\right)^{2}=\frac{d_{t}^{\text {worf }}}{\left(\widehat{\gamma}_{t}+\left|g_{t}\right| \eta_{t}^{\theta} / \psi_{t}^{*}\right)^{2}}+1 .
$$

Then, the value function is

$$
V_{T}^{\text {worf }}(x, f)=\mathrm{E}\left[\widehat{Y}_{0}^{T} \mid F_{0}=f\right] x, \quad(x, f) \in \mathbb{R}_{+} \times \mathbb{R}^{K},
$$

and the time-consistent optimal portfolio is

$$
u_{t}^{*}=\frac{1}{\widehat{\gamma}_{t}+\left|g_{t}\right| \eta_{t}^{\theta} / \psi_{t}^{*}}\left(A\left(F_{t}\right)+\eta_{t}^{V}\left\|A\left(F_{t}\right)\right\| I_{d}\right)^{-1}\left(m_{t}-\frac{c_{t}^{\text {worf }}-\left(\widehat{\gamma}_{t}+\left|g_{t}\right| \eta_{t}^{\theta} / \psi_{t}^{*}\right)}{b_{t}^{\text {worf }}} \mathbf{1}_{d}\right),
$$

for all $0 \leq t \leq T-1$.

The proof of Proposition 7 is in Section A.3. The equation (2.7) is essentially the same as the equation (A11) in Garlappi et al. (2007), so it must have a unique positive solution by Garlappi et al. (2007). We give the details of the proofs of the existence of the unique positive solution to the equation (2.7) in Section A.3.

The main difference between Garlappi et al. (2007) and our model is the covariance matrix. The optimal solution in Garlappi et al. (2007) can be regarded as the case when $\eta_{t}^{V}$ is zero in our solution. In our case, the multiple priors for covariance affects the optimal portfolio.

The division of $\widehat{\gamma}_{t}$ by $X_{t}$ has an important role in the problem without the risk-free asset. Without this division, we can not derive the explicit solution since the solution of the polynomial (2.7) depends on the value of $X_{t}$. This implies that the $t$-step value function $\widehat{Y}_{t}^{T}$, also depends on the value of $X_{t}$. Then, it is difficult to compute the conditional expected value of $\widehat{Y}_{t}^{T}$ given $\mathcal{F}_{t-1}^{R, F}$ under the probability measure $\mathbb{P}^{\theta, V}$. Hence, the trade-off parameter's division by the wealth is crucial.

Now we introduce a short-selling constraint. Let $\widetilde{\mathcal{A}}_{+T}^{u}$ be a set of admissible portfolios with a short-selling constraint such that

$\widetilde{\mathcal{A}}_{+T}^{u}=\left\{u=\left(u_{t}\right)_{t=0}^{T-1} \mid u\right.$ is a $\mathbb{R}^{d}$-valued and $\mathbb{F}^{R, F}$-adapted process, $\mathbf{1}_{d}^{\prime} u_{t}=1$ and $\left.u_{t} \in \mathbb{R}_{+}^{d}\right\}$.

Then, the dynamic optimization problem with a short-selling constraint can be expressed as

$$
\begin{gathered}
V_{+T}^{w o r f}(x, f)=\max _{\left(u_{t}\right)_{t=0}^{T-1} \in \widetilde{\mathcal{A}}_{+T}^{u}\left(\theta_{t}, V_{t}\right)_{t=0}^{T-1} \in \mathcal{A}_{T}^{\theta, V}} \mathrm{E}^{\theta, V}\left[\sum_{t=0}^{T-1} \delta^{t} X_{t} \widehat{c}_{t}\left(F_{t}, \theta_{t}, V_{t}, u_{t}\right)\right] \\
\text { subject to } X_{t+1}=\left(1+\left(R_{t+1}\right)^{\prime} u_{t}\right) X_{t}, \quad t \geq 0, \\
X_{0}=x, \quad F_{0}=f .
\end{gathered}
$$

Then, the following proposition holds. 
Proposition 8 For all $0 \leq t \leq T-1$, let

$$
\begin{aligned}
\widehat{Y}_{T}^{+T} & =0, \\
g_{t}^{+} & =1+\delta \mathrm{E}\left[\widehat{Y}_{t+1}^{+T} \mid \mathcal{F}_{t}^{R, F}\right], \quad m_{t}^{+}=\mathrm{E}\left[\left(1+\delta \widehat{Y}_{t+1}^{+T}\right) \mu\left(F_{t+1}\right) \mid \mathcal{F}_{t}^{R, F}\right], \\
\widehat{Y}_{t}^{+T} & =\max _{u_{t} \in \overline{\mathcal{C}}^{+}} \min _{\left.\left(\theta_{t}, V_{t}\right) \in \mathcal{A}_{T}^{\theta, V}\right|_{t} ^{t}}\left\{g_{t}^{+}+\left(m_{t}^{+}+g_{t}^{+} \theta_{t}\right)^{\prime} u_{t}-\frac{\widehat{\gamma}_{t}}{2} u_{t}^{\prime}\left(A\left(F_{t}\right)+V_{t}\right) u_{t}\right\},
\end{aligned}
$$

where $\overline{\mathcal{C}^{+}}$is a set of portfolios such that

$$
\overline{\mathcal{C}^{+}}=\left\{\phi \in \mathbb{R}_{+}^{d} \mid 1=\mathbf{1}_{d}^{\prime} \phi .\right\} .
$$

Then, the value function is

$$
V_{+T}^{w o r f}(x, f)=\mathrm{E}\left[\widehat{Y}_{0}^{+T} \mid F_{0}=f\right] x,
$$

and the optimal portfolio at time $t$ is

$$
u_{t}^{*}=\arg \max _{u_{t} \in \overline{\mathcal{C}}^{+}} \min _{\left.\left(\theta_{t}, V_{t}\right) \in \mathcal{A}_{T}^{\theta, V}\right|_{t} ^{t}}\left\{g_{t}^{+}+\left(m_{t}^{+}+g_{t}^{+} \theta_{t}\right)^{\prime} u_{t}-\frac{\widehat{\gamma}_{t}}{2} u_{t}^{\prime}\left(A\left(F_{t}\right)+V_{t}\right) u_{t}\right\} .
$$

The proof of Proposition 8 is in Section A.3. The $t$ stage problem,

$$
\max _{u_{t} \in \overline{\mathcal{C}^{+}}} \min _{\left.\left(\theta_{t}, V_{t}\right) \in \mathcal{A}_{T}^{\theta, V}\right|_{t} ^{t}}\left\{g_{t}^{+}+\left(m_{t}^{+}+g_{t}^{+} \theta_{t}\right)^{\prime} u_{t}-\frac{\widehat{\gamma} t}{2} u_{t}^{\prime}\left(A\left(F_{t}\right)+V_{t}\right) u_{t}\right\},
$$

can be rewritten as follows,

$$
\begin{aligned}
& \max _{u_{t} \in \overline{\mathcal{C}^{+}}} \min _{\left.\left(\theta_{t}, V_{t}\right) \in \mathcal{A}_{T}^{\theta, V}\right|_{t} ^{t}}\left\{g_{t}^{+}+\left(m_{t}^{+}+g_{t}^{+} \theta_{t}\right)^{\prime} u_{t}-\frac{\widehat{\gamma}_{t}}{2} u_{t}^{\prime}\left(A\left(F_{t}\right)+V_{t}\right) u_{t}\right\}= \\
& \max _{u_{t} \in \overline{\mathcal{C}}^{+}}\left\{g_{t}^{+}+\left(m_{t}^{+}\right)^{\prime} u_{t}-\frac{\widehat{\gamma}_{t}}{2} u_{t}^{\prime}\left(A\left(F_{t}\right)+\eta_{t}\left\|A\left(F_{t}\right)\right\| I_{d}\right) u_{t}-\left|g_{t}^{+}\right| \eta_{t}^{\theta} \sqrt{u_{t}^{\prime}\left(A\left(F_{t}\right)+\eta_{t}\left\|A\left(F_{t}\right)\right\| I_{d}\right) u_{t}}\right\} .
\end{aligned}
$$

Therefore, the solution to the above problem exists since the above objective is continuous in $u_{t}$.

\section{Limiting Behaviors}

In this section, we study limiting behaviors of optimal portfolios. We have postulated that the parameters $\left(\widehat{\gamma}_{t}\right)_{t=0}^{T-1},\left(\eta_{t}^{\theta}\right)_{t=0}^{T-1}$, and $\left(\eta_{t}^{V}\right)_{t=0}^{T-1}$ are non-negative. We therefore consider the behaviors of the optimal portfolios when these parameters go to infinity.

We first consider the optimal portfolio with the risk-free asset. By Proposition 6, the optimal portfolio with the risk-free asset is

$$
u_{t}^{*}=\frac{1}{\widehat{\gamma}_{t}}\left(1-\frac{\left|g_{t}^{e}\right| \eta_{t}^{\theta}}{\sqrt{a_{t}^{e}}}\right)^{+}\left(A\left(F_{t}\right)+\eta_{t}^{V}\left\|A\left(F_{t}\right)\right\| I_{d}\right)^{-1} m_{t}^{e},
$$

for all $t \geq 0$. Then, $m_{t}^{e}$ and $g_{t}^{e}$ are determined independently of the values of $\widehat{\gamma}_{t}, \eta_{t}^{\theta}$ and $\eta_{t}^{V}$. $a_{t}^{e}$ can be expressed as

$$
\begin{aligned}
a_{t}^{e} & =\left(m_{t}^{e}\right)^{\prime}\left(A\left(F_{t}\right)+\eta_{t}^{V}\left\|A\left(F_{t}\right)\right\| I_{d}\right)^{-1} m_{t}^{e} \\
& =\frac{1}{\eta_{t}^{V}}\left(m_{t}^{e}\right)^{\prime}\left(\frac{1}{\eta_{t}^{V}} A\left(F_{t}\right)+\left\|A\left(F_{t}\right)\right\| I_{d}\right)^{-1} m_{t}^{e},
\end{aligned}
$$


therefore, $a_{t}^{e} \rightarrow 0$ as $\eta_{t}^{V} \rightarrow \infty$. This implies that for any fixed $\widehat{\gamma}_{t}$ and $\eta_{t}^{\theta}$,

$$
\lim _{\eta_{t}^{V} \rightarrow \infty} u_{t}^{*}=\mathbf{0}_{d}
$$

So, if the investor has absolutely no confidence in the estimated conditional variance, she quits investing in the risky assets. Similarly, if $\eta_{t}^{\theta} \rightarrow \infty$, then

$$
\lim _{\eta_{t}^{\theta} \rightarrow \infty}\left(1-\frac{\left|g_{t}^{e}\right| \eta_{t}^{\theta}}{\sqrt{a_{t}^{e}}}\right)^{+}=0
$$

for any fixed $\eta_{t}^{V}$. Therefore, $u_{t}^{*}$ converges to $\mathbf{0}_{d}$ when $\eta_{t}^{\theta}$ tends to infinity. These behaviors are natural since the investor with strong doubts of the correctnesses of the risky assets' parameters prefers investing in the risk-free asset which yields a deterministic return $r_{\mathrm{f}}$. Thus, the effects of multiple priors are similar to the risk-averse behavior if the risk-free asset is present. However, with the absence of risk-free asset, limiting portfolios are two famous portfolios, namely, the global minimum-variance portfolio and the equally weighted portfolio.

\section{Proposition 9}

1. With the risk-free asset. If $\widehat{\gamma}_{t}, \eta_{t}^{\theta}$ or $\eta_{t}^{V}$ tends to infinity, then the investor invests all wealth in the risk-free asset.

2. Without the risk-free asset.

(a) If $\widehat{\gamma}_{t}$ or $\eta_{t}^{\theta}$ tends to infinity and if $\eta_{t}^{V}$ is fixed, then the investor invests in the global minimum-variance portfolio under $\mathbb{P}^{\theta, V}$, that is

$$
\lim _{\eta_{t}^{\theta} \text { or } \widehat{\gamma}_{t} \rightarrow \infty} u_{t}^{*}=\frac{1}{\mathbf{1}_{d}^{\prime}\left(A\left(F_{t}\right)+\eta_{t}^{V}\left\|A\left(F_{t}\right)\right\| I_{d}\right)^{-1} \mathbf{1}_{d}}\left(A\left(F_{t}\right)+\eta_{t}^{V}\left\|A\left(F_{t}\right)\right\| I_{d}\right)^{-1} \mathbf{1}_{d},
$$

when permitting short selling. When short selling is not allowed, then the optimal portfolio again converges to the global minimum-variance portfolio.

$$
\lim _{\eta_{t}^{\theta} \rightarrow \infty} u_{t}^{*}=u_{t}^{+G M V}:=\arg \min _{u \in \mathcal{C}^{+}}\left\{u^{\prime}\left(A\left(F_{t}\right)+\eta_{t}^{V}\left\|A\left(F_{t}\right)\right\| I_{d}\right) u\right\} .
$$

(b) If $\eta_{t}^{V}$ tends to infinity, then the investor invests in the equally weighted portfolio whether short selling is allowed or not, that is,

$$
\lim _{\eta_{t}^{V} \rightarrow \infty} u_{t}^{*}=\frac{1}{d} \mathbf{1}_{d}
$$

Proof . By Proposition 7, the optimal portfolio without the risk-free asset is

$$
u_{t}^{*}=\frac{1}{\widehat{\gamma}_{t}+\left|g_{t}\right| \eta_{t}^{\theta} / \psi_{t}^{*}}\left(A\left(F_{t}\right)+\eta_{t}^{V}\left\|A\left(F_{t}\right)\right\| I_{d}\right)^{-1}\left(m_{t}-\frac{c_{t}^{\text {worf }}-\left(\widehat{\gamma}_{t}+\left|g_{t}\right| \eta_{t}^{\theta} / \psi_{t}^{*}\right)}{b_{t}^{\text {worf }}} \mathbf{1}_{d}\right)
$$

where

$$
\begin{aligned}
& a_{t}^{\text {worf }}=m_{t}^{\prime}\left(A\left(F_{t}\right)+\eta_{t}^{V}\left\|A\left(F_{t}\right)\right\| I_{d}\right)^{-1} m_{t}, \quad c_{t}^{\text {worf }}=m_{t}^{\prime}\left(A\left(F_{t}\right)+\eta_{t}^{V}\left\|A\left(F_{t}\right)\right\| I_{d}\right)^{-1} \mathbf{1}_{d}, \\
& b_{t}^{\text {worf }}=\mathbf{1}_{d}^{\prime}\left(A\left(F_{t}\right)+\eta_{t}^{V}\left\|A\left(F_{t}\right)\right\| I_{d}\right)^{-1} \mathbf{1}_{d}, \quad d_{t}^{\text {worf }}=a_{t}^{\text {worf }} b_{t}^{\text {worf }}-\left(c_{t}^{\text {worf }}\right)^{2}
\end{aligned}
$$


and $\psi_{t}^{*}$ is the unique solution of the following quartic equation,

$$
b_{t}^{\text {worf }}\left(\psi_{t}^{*}\right)^{2}=\frac{d_{t}^{\text {worf }}}{\left(\widehat{\gamma}_{t}+\left|g_{t}\right| \eta_{t}^{\theta} / \psi_{t}^{*}\right)^{2}}+1 .
$$

Similar to the case with the risk-free asset, $m_{t}$ and $g_{t}$ are determined independently of the values of $\widehat{\gamma}_{t}, \eta_{t}^{\theta}$ and $\eta_{t}^{V}$, so we can regard them as constants. To study the limiting behavior of $u_{t}^{*}$, it is important to use the quartic equation for $\psi_{t}^{*}$.

The quartic equation can be transformed as follows:

$$
\left(\widehat{\gamma}_{t}+\frac{\left|g_{t}\right| \eta_{t}^{\theta}}{\psi_{t}^{*}}\right)^{2}=\frac{d_{t}^{\text {worf }}}{b_{t}^{\text {worf }}\left(\psi_{t}^{*}\right)^{2}-1}
$$

The benefit of the above expression is that it separates the terms depending on $\eta_{t}^{\theta}$ and $\eta_{t}^{V}$. In addition,

$$
\left(\widehat{\gamma}_{t}+\frac{\left|g_{t}\right| \eta_{t}^{\theta}}{\psi_{t}^{*}}\right)^{2}=\frac{d_{t}^{\text {worf }}}{b_{t}^{\text {worf }}\left(\psi_{t}^{*}\right)^{2}-1} \geq \widehat{\gamma}_{t}^{2}>0
$$

for all $\widehat{\gamma}_{t}, \eta_{t}^{\theta}$, and $\eta_{t}^{V}$. For any fixed $\widehat{\gamma}_{t}$ and $\eta_{t}^{V}$, two limits of the equation (3.1) as $\eta_{t}^{\theta} \rightarrow \infty$ can be expected. One is some positive constant $C>0$. Then, we have

$$
\lim _{\eta_{t}^{\theta} \rightarrow \infty}\left(\widehat{\gamma}_{t}+\frac{\left|g_{t}\right| \eta_{t}^{\theta}}{\psi_{t}^{*}}\right)^{2}=\lim _{\eta_{t}^{\theta} \rightarrow \infty} \frac{d_{t}^{\text {worf }}}{b_{t}^{\text {worf }}\left(\psi_{t}^{*}\right)^{2}-1}=C .
$$

The right equality follows that

$$
\lim _{\eta_{t}^{\theta} \rightarrow \infty} \psi_{t}^{*}=C_{1}
$$

for some constant $C_{1}>0$. However, this implies

$$
\lim _{\eta_{t}^{\theta} \rightarrow \infty}\left(\widehat{\gamma}_{t}+\frac{\left|g_{t}\right| \eta_{t}^{\theta}}{\psi_{t}^{*}}\right)^{2}=\infty
$$

Hence it is a contradiction. Therefore, the equation (3.1) diverges when $\eta_{t}^{\theta}$ tends to infinity. Indeed,

$$
\lim _{\eta_{t}^{\theta} \rightarrow \infty} \frac{d_{t}^{\text {worf }}}{b_{t}^{\text {worf }}\left(\psi_{t}^{*}\right)^{2}-1}=\infty
$$

implies that $\left(\psi_{t}^{*}\right)^{2} \rightarrow 1 / b_{t}^{\text {worf }}$. Then,

$$
\lim _{\eta_{t}^{\theta} \rightarrow \infty}\left(\widehat{\gamma}_{t}+\frac{\left|g_{t}\right| \eta_{t}^{\theta}}{\psi_{t}^{*}}\right)^{2}=\infty .
$$

This is consistent. Therefore, the limiting portfolio with respect to $\eta_{t}^{\theta}$ is

$$
\lim _{\eta_{t}^{\theta} \rightarrow \infty} u_{t}^{*}=\frac{1}{b_{t}^{\text {worf }}}\left(A\left(F_{t}\right)+\eta_{t}^{V}\left\|A\left(F_{t}\right)\right\| I_{d}\right)^{-1} \mathbf{1}_{d} .
$$

This limiting portfolio is the global minimum-variance portfolio under $\mathbb{P}^{\theta, V}$.

We consider the limiting behavior with respect to $\eta_{t}^{V} \cdot d_{t}^{\text {worf }}$ and $b_{t}^{\text {worf }}$ can be expressed as

$$
\begin{aligned}
& d_{t}^{\text {worf }}=\frac{1}{\left(\eta_{t}^{V}\right)^{2}} d_{t}^{\eta}, \quad \lim _{\eta_{t}^{V} \rightarrow \infty} d_{t}^{\eta}=d_{0}>0, \\
& b_{t}^{\text {worf }}=\frac{1}{\eta_{t}^{V}} b_{t}^{\eta}, \quad \lim _{\eta_{t}^{V} \rightarrow \infty} b_{t}^{\eta}=b_{0}>0 .
\end{aligned}
$$


Then, the RHS in the equation (3.1) becomes

$$
\frac{d_{t}^{\text {worf }}}{b_{t}^{\text {worf }}\left(\psi_{t}^{*}\right)^{2}-1}=\frac{d_{t}^{\eta}}{b_{t}^{\eta} \eta_{t}^{V}\left(\left(\psi_{t}^{*}\right)^{2}-\eta_{t}^{V} / b_{t}^{\eta}\right)}
$$

We first assume that the LHS in the equation (3.1) diverges, that is,

$$
\lim _{\eta_{t}^{V} \rightarrow \infty}\left(\widehat{\gamma}_{t}+\frac{\left|g_{t}\right| \eta_{t}^{\theta}}{\psi_{t}^{*}}\right)^{2}=\infty
$$

This implies that $\psi_{t}^{*}$ converges to 0 when $\eta_{t}^{V} \rightarrow \infty$. However, for the RHS in the equation $(3.1)$,

$$
\lim _{\eta_{t}^{V} \rightarrow \infty} \frac{d_{t}^{\eta}}{b_{t}^{\eta} \eta_{t}^{V}\left(\left(\psi_{t}^{*}\right)^{2}-\eta_{t}^{V} / b_{t}^{\eta}\right)}=0 .
$$

This is a contradiction. It follows that there exists some positive constant $C_{2}>0$ such that

$$
\lim _{\eta_{t}^{V} \rightarrow \infty} \frac{d_{t}^{\eta}}{b_{t}^{\eta} \eta_{t}^{V}\left(\left(\psi_{t}^{*}\right)^{2}-\eta_{t}^{V} / b_{t}^{\eta}\right)}=C_{2}
$$

Then, $\eta_{t}^{V}\left(\left(\psi_{t}^{*}\right)^{2}-\eta_{t}^{V} / b_{t}^{\eta}\right) \rightarrow C_{3}$ for some positive constant $C_{3}>0$ as $\eta_{t}^{V} \rightarrow \infty$. This implies that $\psi_{t}^{*}$ diverges. Then the LHS in the equation (3.1) also converges to $\widehat{\gamma}_{t}^{2}$, so it is consistent with our expectation.

By the definition, it holds that

$$
\lim _{\eta_{t}^{V} \rightarrow \infty} \frac{c_{t}^{\text {worf }}}{b_{t}^{\text {worf }}}=C_{4}
$$

for some constant $C_{4}$. Furthermore, we have

$$
\lim _{\eta_{t}^{V} \rightarrow \infty}\left(A\left(F_{t}\right)+\eta_{t}^{V}\left\|A\left(F_{t}\right)\right\| I_{d}\right)^{-1} x=\mathbf{0}_{d}
$$

for any $x \in \mathbb{R}^{d}$. Moreover, $\widehat{\gamma}_{t}+\left|g_{t}\right| \eta_{t}^{\theta} / \psi_{t}^{*}$ converges to $\widehat{\gamma}_{t}$ as $\eta_{t}^{V} \rightarrow \infty$. Hence,

$$
\lim _{\eta_{t}^{V} \rightarrow \infty} \frac{1}{\widehat{\gamma}_{t}+\left|g_{t}\right| \eta_{t}^{\theta} / \psi_{t}^{*}}\left(A\left(F_{t}\right)+\eta_{t}^{V}\left\|A\left(F_{t}\right)\right\| I_{d}\right)^{-1}\left(m_{t}-\frac{c_{t}^{\text {worf }}}{b_{t}^{\text {worf }}} \mathbf{1}_{d}\right)=\mathbf{0}_{d} .
$$

On the other hand,

$$
\lim _{\eta_{t}^{V} \rightarrow \infty} \frac{\left(A\left(F_{t}\right)+\eta_{t}^{V}\left\|A\left(F_{t}\right)\right\| I_{d}\right)^{-1} \mathbf{1}_{d}}{b_{t}^{\text {worf }}}=\lim _{\eta_{t}^{V} \rightarrow \infty} \frac{\left(A\left(F_{t}\right) / \eta_{t}^{V}+\left\|A\left(F_{t}\right)\right\| I_{d}\right)^{-1} \mathbf{1}_{d}}{\mathbf{1}_{d}^{\prime}\left(A\left(F_{t}\right) / \eta_{t}^{V}+\left\|A\left(F_{t}\right)\right\| I_{d}\right)^{-1} \mathbf{1}_{d}}=\frac{1}{d} \mathbf{1}_{d} .
$$

Finally, it holds that

$$
\lim _{\eta_{t}^{V} \rightarrow \infty} u_{t}^{*}=\frac{1}{d} \mathbf{1}_{d}
$$

Hence, the limiting portfolio as $\eta_{t}^{V} \rightarrow \infty$ is the equally weighted portfolio.

In the case when short selling is not allowed, it holds that $u_{t}^{*} \rightarrow \mathbf{1}_{d} / d$ as $\eta_{t}^{V} \rightarrow \infty$ since $\mathbf{1}_{d} / d$ is an interior point of $\overline{\mathcal{C}^{+}}$. When $\eta_{t}^{\theta} \rightarrow \infty$,

$$
\lim _{\eta_{t}^{\theta} \rightarrow \infty} u_{t}^{*}=u_{t}^{+G M V}=\arg \min _{u \in \overline{\mathcal{C}^{+}}}\left\{u^{\prime}\left(A\left(F_{t}\right)+\eta_{t}^{V}\left\|A\left(F_{t}\right)\right\| I_{d}\right) u\right\}
$$


since the objective function is proportional to

$$
\frac{1}{\eta_{t}^{\theta}}\left(m_{t}^{+}\right)^{\prime} u_{t}-\frac{\widehat{\gamma}_{t}}{2 \eta_{t}^{\theta}} u_{t}^{\prime}\left(A\left(F_{t}\right)+\eta_{t}^{V}\left\|A\left(F_{t}\right)\right\| I_{d}\right) u_{t}-\left|g_{t}^{+}\right| \sqrt{u_{t}^{\prime}\left(A\left(F_{t}\right)+\eta_{t}^{V}\left\|A\left(F_{t}\right)\right\| I_{d}\right) u_{t}}
$$

and the effects of the first two terms become small when $\eta_{t}^{\theta}$ becomes large.

Interestingly, the optimal portfolio $u_{t}^{*}$ also converges to the equally weighted portfolio when $\eta_{t}^{V} \rightarrow \infty$ and $\eta_{t}^{\theta}=0$. This means that the investor chooses the equally weighted portfolio under the strong uncertainty for variance even if she believes that the errors of the estimated conditional expected returns do not exist. Therefore, we conclude that the uncertainty about variances has a stronger impact on the investor's portfolio selection than the uncertainty about means.

However, the above result slightly differs from natural intuition. The readers may think that the investor tends to choose the asset having the highest expected return if the degree of suspicion for variances $\eta_{t}^{V}$, is sufficiently large. This question can be resolved through the inequality constraint (2.3). If $\eta_{t}^{V}$ is sufficiently large, then the error of variance $V_{t}$, is also large. This implies that

$$
\theta_{t}^{\prime}\left(A\left(F_{t}\right)+V_{t}\right)^{-1} \theta_{t}
$$

is very close to 0 . Then, the mean errors $\theta_{t}$ in the investor's belief can take an arbitrary value if $\eta^{V} \rightarrow \infty$. Therefore, the investor's worst scenario is that the means take large negative values which are the same across all risky assets. Hence, the investor chooses the equally weighted portfolio if $\eta^{V} \rightarrow \infty$.

Without the risk-free asset, the limiting case $\eta_{t}^{\theta} \rightarrow \infty$ is the same as the special case of the 2-norm-constrained optimal portfolio in DeMiguel et al. (2009a). However, our framework admits dynamic changes of covariance, so the conditional covariance matrix appears in the limiting portfolio, whereas the unconditional covariance matrix appears in DeMiguel et al. (2009a) since they consider the static optimization.

Unfortunately, without the risk-free asset, the limiting strategies are not applicable straightforwardly since the value functions diverge. These limiting portfolios are only admissible in the initial period of the investment horizon. However, if $\eta_{t}^{\theta}$ and $\eta_{t}^{V}$ are sufficiently large, then the optimal portfolios are very similar to the limiting portfolios, so we can use the optimal portfolios with large $\eta_{t}^{\theta}$ and $\eta_{t}^{V}$ as proxies of the limiting portfolios. In Section 4, we study the cases of large $\eta_{t}^{\theta}$ and $\eta_{t}^{V}$.

Remark 10 As mentioned below, in a limiting case, Pflug et al. (2012) reach essentially the same conclusion as us, although there are several differences. Pflug et al. (2012) show that as a model uncertainty measured by the Kantorovich metric increases, an optimal portfolio in a mean-standard-deviation optimization problem converges to the equally weighted portfolio. Specifically, Pflug et al. (2012) consider the following problem: ${ }^{2}$

$$
\begin{aligned}
\max _{u} \min _{\mathbb{Q}: d_{2}(\mathbb{P}, \mathbb{Q})} \leq \kappa & \left\{\mathrm{E}^{\mathbb{Q}}[X]-\frac{\gamma}{2} \sqrt{\operatorname{Var}^{\mathbb{Q}}(X)}\right\}, \\
\text { subject to } X & =R^{\prime} u, \\
1 & =\mathbf{1}_{d}^{\prime} u,
\end{aligned}
$$

where $d_{2}$ is the Kantorovich metric with order 2 , and $R$ is a risky assets' return vector. $\gamma$ is a non-negative trade-off parameter between returns and risks. $\mathrm{E}^{\mathbb{Q}}$ and $\operatorname{Var}^{\mathbb{Q}}$ are expectation and

\footnotetext{
${ }^{2}$ We modify the original problem in Pflug et al. (2012) for this to take the same form as in this paper. It can be easily seen that the problem (3.3) is equivalent to the original problem in Pflug et al. (2012).
} 
variance operators under a probability measure $\mathbb{Q}$, respectively. $\kappa$ is a non-negative constant that represents a degree of uncertainty. Pflug et al. (2012) show that the solution to the problem (3.3) converges to the equally weighted portfolio as $\kappa \rightarrow \infty$.

One of the differences between our model and the model in Pflug et al. (2012) is the measure of uncertainty. Pflug et al. (2012) adopts the Kantorovich metric, while it is a mathematically sophisticated concept of a distance among probability measures, it is usually hard to compute, and it is not usually used in practice. In contrast, our measures of uncertainty are a confidence interval around means and a relative error of covariances, which are widely used in practice and easy to compute.

Another difference between our model and the model in Pflug et al. (2012) is the objective function. Let us assume $T=1$ in our model, so we consider a static problem. In order to apply the approach of Pflug et al. (2012) to our objective function, we need to replace the term of the standard deviation in (3.3) to the variance, that is

$$
\begin{aligned}
\max _{u} \min _{\mathbb{Q}: d_{2}(\mathbb{P}, \mathbb{Q}) \leq \kappa} & \left\{\mathrm{E}^{\mathbb{Q}}[X]-\frac{\gamma}{2} \operatorname{Var}^{\mathbb{Q}}(X)\right\}, \\
\text { subject to } X & =R^{\prime} u \\
1 & =\mathbf{1}_{d}^{\prime} u
\end{aligned}
$$

However, since the objective function in the problem (3.4) does not satisfy the assumptions in Proposition 1 in Pflug et al. (2012), we can not use the results of Pflug et al. (2012). Therefore, the solution to the problem (3.4) may not converge to the equally weighted portfolio. For details about this discussion, we also refer to Wozabal (2014).

Considering a unique prior case (i.e., $\kappa=0$ ), we can easily show that the solution to the problem (3.3) is proportional to the solution to the problem (3.4). However, the trade-off parameter $\gamma$ has different implications in these problems. As seen in Section 2, in our model, we can regard $\gamma$ as a coefficient of (absolute) risk aversion. On the other hand, economic implication of $\gamma$ in the problem (3.3) is not clear.

Finally, we adopt dynamic mean-variance optimization, whereas the model in Pflug et al. (2012) is static. Therefore, our model allows an investment strategy that changes portfolios in reaction to the state variable $F_{t}$. In Section 4, we will see that this investment strategy often performs well in practice.

\section{Optimality of Naive Investment Strategies in Back Tests}

In this section, we conduct back tests of dynamic optimization with multiple priors. We use the following three data sets.

1. International Equity Indexes. The four MSCI indexes: US, Japan, UK, and Germany. Each index is month-end US-dollar valued. The data source is the Thomson Reuters Datastream.

2. Industry Indexes. The five monthly returns of industry indexes in the US stock market: Consumer, Manufacture, HiTechnology, Health, and Others. The data source is the Kenneth French's web site.

3. Size- and Value-Sorted Portfolios. The six monthly returns of the $2 \times 3$ size- and book-to-market-sorted portfolios in the US stock market by Fama and French (1993). The data source is the Kenneth French's web site. 
We also use monthly returns of 90-day US treasury bill from the Thomson Reuters Datastream as risk-free rates. Each dataset consists of monthly returns from January 1975 to December 2014. In order to focus on the diversification within the risky assets, we consider the asset allocations without the risk-free asset and compare out-of-sample Sharpe ratios.

\subsection{Methodology of the Back Tests}

In each dataset, we assume that the return vector is modeled by a two-state, recursive and time-homogeneous Markov regime-switching model, such that

$$
R_{t+1}=\mu\left(F_{t+1}\right)+\sigma\left(F_{t+1}\right) \epsilon_{t+1}, \quad t \geq 0,
$$

where $\left(F_{t}\right)_{t=0}^{T}$ is a two-state, recursive and time-homogeneous Markov chain. $\left(F_{t}\right)_{t=0}^{T}$ and $\left(\epsilon_{t}\right)_{t=1}^{T}$ are mutually independent. The transition probability matrix of $\left(F_{t}\right)_{t=0}^{T}$ is constant over time by the time-homogeneity of $\left(F_{t}\right)_{t=0}^{T}$. This model satisfies Assumption 1.

We assume that one of the states of $F_{t}$ is a "good state" and another state is a "bad state". Whether the market condition is good or bad is determined by the values of conditional means and variances of the return vectors. Consider the state in which more than half of means and reciprocals of variances are larger than those in another state. We call this state the "good state" and call the other state the "bad state". If these values are the same, as the good state we choose the state in which the sum of the marginal conditional means is larger than that in the other state.

We consider two types of investors. The first type assumes that the returns of the indexes are not regime switching. This type of investor always considers the mean and variance of the returns are constants. We call this type "IID". The second type assumes that the returns are driven by the Markov chain $\left(F_{t}\right)_{t=0}^{T}$. Hence, this type of investor assumes that the conditional mean and variance of the returns vary over the time. We call this type "RS". The IID and $\mathrm{RS}$ investors compute their portfolios using the dynamic optimization without the risk-free asset proposed in Propositions 7 and 8.

As for the preference parameters, $\widehat{\gamma}_{t}$ and $\delta$ are constant over time and states. We set $\widehat{\gamma}_{t}=1$ and $\delta=0.99$. To study the effect of the multiple priors, we consider six different values of $\left(\eta_{t}^{\theta}=0,1,2,3,4,5\right)$ and five different values of $\left(\eta_{t}^{V}=0.0,0.5,1.0,3.0,5.0\right)$. These parameters are fixed over time and states. Furthermore, we also consider two different cases: the one in which short selling is permitted and the one in which it is not permitted. Therefore, we compute $6 \times 5 \times 2$ portfolios for each investor type. The large $\eta_{t}^{\theta}$ and $\eta_{t}^{V}$ represent strong degrees of suspicion of investors, so we can compare the investors with different levels of suspicion. As benchmarks, we also consider the equally weighted portfolio (EW), the portfolio maximizing the single-period empirical Sharpe ratio $(\max \mathrm{SR}$ ), and the single-period global minimumvariance portfolio (GMV). In addition, we use the value-weighted portfolio (VW) as one of the benchmarks for the industry indexes and the size- and value-sorted portfolios data set ${ }^{3}$. Note that the IID investor's max SR at time $t$ maximizes the following objective function:

$$
f_{\mathrm{IID}}(u):=\frac{\bar{\mu}^{\prime} u-r_{\mathrm{f}, t}}{\sqrt{u^{\prime} \bar{\Sigma} u}}
$$

where $\bar{\mu}$ and $\bar{\Sigma}$ are sample mean and variance of returns and $r_{\mathrm{f}, t}$ is the risk-free rate at time $t$. On the other hand, the RS investor's max SR at time $t$ with the state $F_{t}=i$ maximizes the following objective function,

$$
f_{\mathrm{RS}}(u):=\frac{\bar{\mu}_{i}^{\prime} u-r_{\mathrm{f}, t}}{\sqrt{u^{\prime}\left(\bar{\sigma}_{i} \bar{\sigma}_{i}^{\prime}\right) u}}
$$

\footnotetext{
${ }^{3}$ Unfortunately, we can not obtain the data of the market values of the US dollar-based MSCI indexes. So, we compute the value-weighted portfolio only for the abovementioned two data sets
} 
where $\bar{\mu}_{i}$ and $\bar{\sigma}_{i} \bar{\sigma}_{i}^{\prime}$ are estimated mean and variance of returns at state $i$. By the above definition, max SR delivers the largest Sharpe ratio without estimation errors.

Furthermore, we consider the following six extreme strategies. In these six strategies, $\eta_{t}^{\theta}$ and $\eta_{t}^{V}$ take different values in the different states of $F_{t}$. The first strategy is "GMV in the good state and EW in the bad state" (GMV-EW). Under this strategy, the investor invests in the global minimum-variance portfolio in the state of good market condition and in the equally weighted portfolio in the state of bad market condition. This strategy can be interpreted as the optimal portfolio when $\eta_{t}^{V}=0$ and $\eta_{t}^{\theta}$ tends to infinity in the good state and when $\eta_{t}^{V}$ tends to infinity in the bad state. The second strategy is "EW in the good state and No Error in the bad state" (EW-NE). Under this strategy, the investor invests in the equally weighted portfolio in the good state and in the optimal portfolio of Proposition 7 and 8 with $\eta_{t}^{\theta}=\eta_{t}^{V}=0$ in the bad state. This strategy can be interpreted as the optimal portfolio when $\eta_{t}^{V}=0$ and $\eta_{t}^{\theta}$ tends to infinity in the good state and when $\eta_{t}^{\theta}$ and $\eta_{t}^{V}$ are 0 in the bad state. The third strategy is "GMV in the good state and No Error in the bad state" (GMV-NE). Under this strategy, the investor invests in the global minimum-variance portfolio in the good state and in the optimal portfolio with $\eta_{t}^{\theta}=\eta_{t}^{V}=0$ in the bad state. This strategy can be interpreted as the optimal portfolio when $\eta_{t}^{V}=0$ and $\eta_{t}^{\theta}$ tends to infinity in the good state and when $\eta_{t}^{\theta}$ and $\eta_{t}^{V}$ are 0 in the bad state. The rest of the strategies are the reverse strategies of the above three strategies "EW in the good state and GMV in the bad state" (EW-GMV), "No Error in the good state and EW in the bad state" (NE-EW), and "No Error in the good state and GMV in the bad state" (NE-GMV). The abbreviations and the six extreme strategies are summarized in Table 1.

In the back tests, each investor needs to estimate the distribution parameters from the data. The rolling window of estimation is fixed to 240 months. At each time, the IID investor computes the sample mean and variance of the data over the past 240 months from current time. Similarly, the RS investor estimates the distribution parameters using the data over the past 240 months from current time. The RS investor estimates the parameters by the EM algorithm proposed by Hamilton (1990). At the time when $t$ months have passed after the start of investment, the investors compute the optimal plans of the portfolios for $240-t$ periods and invest in their optimal portfolios in the initial period. For example, suppose that the investors would like to decide the portfolios in January 2000. They first compute the optimal portfolio plans for 180 months, from January 2000 to December 2014. Then, they invest in the portfolios in the initial period in January 2000.

However, the RS investors can not determine their portfolios since the state variable $F$ in actual data is not observable. To determine their portfolios, they regard the state having the highest conditional probability as the current state, that is, the current state at time $t$ is the following $i_{t}$,

$$
i_{t}=\arg \max _{i \in\{1,2\}} \mathbb{P}\left(F_{t} \text { is in the } i \text { th state. } \mid R_{1}, R_{2}, \ldots, R_{t}\right),
$$

where $R_{s}$ is the return vector of the indexes at time $s=1, \ldots, t$.

The optimality of the portfolios derived in Proposition 7 and 8 are not guaranteed in the above rolling-window approach. However, in general, investors usually choose their portfolios based on the latest information. Moreover, the existing literature (e.g., Garlappi et al. (2007) and DeMiguel et al. (2009b)) adopts the rolling-window approach. For these reasons, we also use this approach.

\subsection{Results of the Back Tests}

Tables 2, 3, and 4 display the out-of-sample Sharpe ratios obtained from the back tests. In all data sets, the extreme strategies tend to deliver larger Sharpe ratios. 
In the international diversification (Table 2), max SR has larger Sharpe ratios than the other typical portfolios, GMV and EW, in all cases (whether the investor type is IID or RS and with or without permitting short selling). When permitting short selling, max SR of the IID investor has the largest Sharpe ratio (0.1014) except for the extreme strategies. However, the IID investor's optimal portfolio with $\eta_{t}^{\theta}=0.0$ and $\eta_{t}^{V}=5.0$ when not permitting short selling delivers the largest Sharpe ratio (0.1081) among all portfolios. Also, the Sharpe ratios when short selling is not permitted tend to be larger than those when it is permitted. This is consistent with the well-known results of Jagannathan and Ma (2003), which states that a short-selling constraint can improve the investment performance.

According to Table 2, the two extreme strategies, GMV-EW and GMV-NE, have good performances whether short selling is permitted or not. GMV-EW with short selling delivers the largest Sharpe ratio (0.1032) among the portfolios with short selling. On the other hand, GMV-NE without short selling has the second largest Sharpe ration (0.1078) among all portfolios. Furthermore, the Sharpe ratios of GMV-EW without short selling and GMV-NE with short selling, 0.0922 and 0.0891 respectively, are not small. Among the typical portfolios (max SR, GMV, and EW) and the typical extreme strategies (GMV-EW and EW-GMV), GMV-EW when permitting short selling has the largest Sharpe ratio. Taking into account the simplicity of computation, GMV-EW when permitting short selling works effectively.

Table 3 reports the results of the industry indexes data set. According to Table 3, GMV has larger Sharpe ratio than max SR, EW and VW in all cases. Precisely, the IID investor's GMV with short selling has the largest Sharpe ratio among all portfolios. Among portfolios without short selling, the RS investor's GMV has the largest Sharpe ratio. However, the two extreme strategies, GMV-EW and EW-GMV, are not so bad either. Both of GMV-EW and EW-GMV defeat max SR, EW, and VW regardless of whether short selling is permitted or not. Comparing GMV-EW and EW-GMV, EW-GMV performs better than GMV-EW. When short selling is permitted, the Sharpe ratio of GMV-EW is 0.2234 , whereas the Sharpe ratio of EW-GMV is 0.2564 . Without short selling, the Sharpe ratio of GMV-EW is 0.2281, whereas the Sharpe ratio of EW-GMV is 0.2473 .

Table 4 reports the results of the size- and value-sorted portfolios data set. The IID investor's optimal portfolio with $\eta_{t}^{\theta}=1.0$ and $\eta^{V}=0.0$ when permitting short selling has the largest Sharpe ratio (0.3406) among all portfolios. On the other hand, similar to the industry indexes data set, GMV has a larger Sharpe ratio than max SR, EW, and VW in all cases. Furthermore, regardless of whether permitting short selling or not, GMV-EW defeats max SR, EW, VW, and GMV, except for the IID investor's GMV. Precisely, GMV-EW has the largest Sharpe ratio (0.2336) among portfolios without short selling.

In summary, GMV-EW and GMV tend to perform well in all data sets. DeMiguel et al. (2009b) report relatively good performances of GMV, so our results are consistent with their results. Unlike DeMiguel et al. (2009b), EW is less efficient in our data sets. However, GMVEW delivers larger Sharpe ratio in the international indexes data set when short selling is permitted and in the size- and value-sorted portfolios data set when short selling is not permitted. Furthermore, GMV-EW defeats max SR in the industry indexes data set regardless of whether permitting short selling or not. Therefore, EW is efficient under particular situations and GMV-EW effectively uses the efficiency of EW.

Note that the IID investor's max SRs deliver larger Sharpe ratios than the RS investor's $\max \mathrm{SRs}$ in all cases. One explanation of this result is the difference between the objective functions. The RS investor's max SR maximizes the conditional Sharpe ratio whereas the IID investor's max SR maximizes the unconditional Sharpe ratio. Since our back tests compute out-of-sample Shape ratios, which are sample analogs of the unconditional Sharpe ratio, it is not surprising that the IID investor's max SRs perform better than the RS investor's max SRs. 
As seen in Section 3, the extreme strategies can not be justified by our framework since the value functions also diverge. However, optimal portfolios with sufficiently large $\eta_{t}^{\theta}$ and $\eta_{t}^{V}$ will work as extreme strategies. We show behaviors of the optimal portfolios with sufficiently large $\eta_{t}^{\theta}$ and $\eta_{t}^{V}$.

We consider the following metrics from the optimal portfolios of EW and GMV:

$$
\left\|u_{t}^{*}\right\|_{G M V}:=\left\|u_{t}^{*}-u_{t}^{G M V}\right\|, \quad\left\|u_{t}^{*}\right\|_{E W}:=\left\|u_{t}^{*}-\mathbf{1}_{d} / d\right\|,
$$

where $u_{t}^{*}$ is the optimal portfolio at time $t$. These metrics represent the root square errors from EW and GMV, so we call these the portfolio errors from EW and GMV. Figure 1 shows the portfolio errors of the international indexes data set from EW and GMV in the case when short selling is permitted. From the upper figures in Figure 1, we see that the high $\eta_{t}^{\theta}$ reduces the portfolio errors from GMV for both IID and RS investors. Similarly, the lower figures in Figure 1 show that the high $\eta_{t}^{V}$ reduces the portfolio errors from $\mathrm{EW}$ for the both investors ${ }^{4}$. This observation is consistent with our theoretical results in Section 3.

Figure 1 suggests that the optimal portfolios with sufficiently large $\eta_{t}^{\theta}$ and $\eta_{t}^{V}$ can be good proxies for the extreme strategies. We try to approximate GMV-EW and EW-GMV by these optimal portfolios. We denote these approximated portfolios by mGMV-EW and mEW-GMV. The first letter $m$ means mimicking extreme strategy.

Table 5 reports the Sharpe ratios of the optimal portfolios with sufficiently large $\eta_{t}^{\theta}$ and $\eta_{t}^{V}$. The second, third, fourth, and fifth columns are the specific values of $\eta_{t}^{\theta}$ and $\eta_{t}^{V}$ of the mimicking strategies. In Table 5, the averages of the root square errors of the mimicking strategies from the (original) extreme strategies are sufficiently small in all the data sets. This implies that the mimicking strategies are good proxies for the extreme strategies. Consequently, the mimicking strategies' Sharpe ratios are close to the original extreme strategies' Sharpe ratios. Hence, we conclude that the investors can asymptotically justify the extreme strategies and exploit the portfolio selections that are extremely robust to the estimation errors.

GMV-EW and GMV-EW are very simple strategies, which means investing in the global minimum-variance portfolio or the equally weighted portfolio with respect to the market condition. These strategies seem to be naive and ad hoc, but they are one of the results of the plausible portfolio optimization; For GMV-EW, the investor assumes that the estimates of the conditional mean in economic booming and the conditional variance in recession are not credible at all, whereas for EW-GMV, she assumes that the estimated variances in economic booming and the estimated means in recession are not credible. Therefore, the investor chooses GMV-EW or EW-GMV as the extremely robust portfolio to estimation errors.

\section{Conclusion}

In this paper, we derive the optimal portfolios in the dynamic mean-variance problems with multiple priors. Furthermore, we show that the optimal portfolios include the equally weighted portfolio and the global minimum-variance portfolio in the limiting cases.

In the back tests, we find that the extreme strategies, especially GMV-EW tends to be relatively mean-variance efficient in the various data sets. In addition, the portfolios with sufficiently large $\eta_{t}^{\theta}$ and $\eta_{t}^{V}$ can be good proxies for the extreme strategies. Therefore, we can mathematically justify the extreme strategies although the extreme strategies seem to be naive; the reason why the investors choose the extreme strategies is that the investors have strong suspicion of the estimated expected return and covariance.

\footnotetext{
${ }^{4}$ In the lower figures in Figure 1, we fix $\eta_{t}^{\theta}=5$. Since the portfolio errors from EW with $\eta_{t}^{\theta}=1$ is larger than those with $\eta_{t}^{\theta}=5$, we do not report the results of $\eta_{t}^{\theta}=1$
} 
Our analysis has an important implication about asset pricing. As seen above, the standard mean-variance analysis does not work in practice, neither does the capital asset pricing model. However, according to our framework, two mean-variance inefficient in theory, and naive portfolios, the global minimum portfolio and the equally weighted portfolio, can be justified as rational selections of the investors. Thus, it is possible that the two portfolios develop asset pricing models. Then, our framework can be used to analyze the rational reasoning behind the investors' choices of these portfolios.

\section{A Appendix}

\section{A.1 Proof of Lemma 4}

Proof of Lemma 4. We first prove that

$$
\mathrm{E}\left[\exp \left\{u^{\prime} R_{t+1}\right\} \zeta_{t+1} \mid \mathcal{F}_{t}^{R, F}, F_{t+1}\right]=\exp \left\{u^{\prime}\left(\mu\left(F_{t+1}\right)+\theta_{t}\right)+\frac{1}{2} u^{\prime}\left(\sigma\left(F_{t+1}\right)\left(\sigma\left(F_{t+1}\right)\right)^{\prime}+V_{t}\right) u\right\}
$$

for all $t \geq 0$ and $u \in \mathbb{R}^{d}$. To simplify the notations, we write $\mu_{t+1}=\mu\left(F_{t+1}\right)$ and $\sigma_{t+1}=$ $\sigma\left(F_{t+1}\right)$. For any fixed constant vector $u \in \mathbb{R}^{d}$, we have

$$
\begin{aligned}
& \mathrm{E} {\left[\exp \left\{u^{\prime} R_{t+1}\right\} \zeta_{t+1} \mid \mathcal{F}_{t}^{R, F}, F_{t+1}\right] } \\
&= \int_{\mathbb{R}^{d}} \frac{1}{(2 \pi)^{d / 2}} \frac{1}{\sqrt{\operatorname{det}\left(\sigma_{t+1} \sigma_{t+1}^{\prime}+V_{t}\right)}} \exp \left\{-\frac{1}{2} \theta_{t}^{\prime}\left(\sigma_{t+1} \sigma_{t+1}^{\prime}+V_{t}\right)^{-1} \theta_{t}+u^{\prime} \mu_{t+1}\right. \\
&+\left(\left(\sigma_{t+1} \sigma_{t+1}^{\prime}+V_{t}\right)^{-1} \theta_{t}+u\right)^{\prime} \sigma_{t+1} \epsilon_{t+1}+\frac{1}{2} \epsilon_{t+1}^{\prime}\left(I_{d}-\sigma_{t+1}^{\prime}\left(\sigma_{t+1} \sigma_{t+1}^{\prime}+V_{t}\right)^{-1} \sigma_{t+1}\right) \epsilon_{t+1} \\
&\left.-\frac{1}{2} \epsilon_{t+1}^{\prime} \epsilon_{t+1}\right\} \mathrm{d} \epsilon_{t+1} \\
&= \exp \left\{u^{\prime}\left(\mu_{t+1}+\theta_{t}\right)+\frac{1}{2} u^{\prime}\left(\sigma_{t+1} \sigma_{t+1}^{\prime}+V_{t}\right) u\right\} \\
& \times \int_{\mathbb{R}^{d}} \frac{1}{(2 \pi)^{d / 2}} \frac{1}{\sqrt{\operatorname{det}\left(\sigma_{t+1} \sigma_{t+1}^{\prime}+V_{t}\right)}} \\
& \times \exp \left\{-\frac{1}{2}\left(\sigma_{t+1} \epsilon_{t+1}-\operatorname{mean}_{t+1}\right)^{\prime}\left(\sigma_{t+1} \sigma_{t+1}^{\prime}+V_{t}\right)^{-1}\left(\sigma_{t+1} \epsilon_{t+1}-\text { mean }_{t+1}\right)\right\} \mathrm{d} \epsilon_{t+1},
\end{aligned}
$$

where mean $_{t+1}=\theta_{t}+\left(\sigma_{t+1} \sigma_{t+1}^{\prime}+V_{t}\right) u$. Then, the above integral is equal to 1 and the equation (A.1) holds.

If $u=\mathbf{0}_{d}$, we have

$$
\mathrm{E}\left[\zeta_{t+1} \mid \mathcal{F}_{t}^{R, F}, F_{t+1}\right]=\mathrm{E}\left[\exp \left\{\mathbf{0}_{d}^{\prime} R_{t+1}\right\} \zeta_{t+1} \mid \mathcal{F}_{t}^{R, F}, F_{t+1}\right]=1 .
$$

Then, the process $\left(Z_{t}\right)_{t=0}^{T}$ is a martingale with respect to $\mathbb{F}^{R, F}$ since

$$
\mathrm{E}\left[Z_{t+1} \mid \mathcal{F}_{t}^{R, F}\right]=\mathrm{E}\left[Z_{t} E\left[\zeta_{t+1} \mid \mathcal{F}_{t}^{R, F}, F_{t+1}\right] \mid \mathcal{F}_{t}^{R, F}\right]=Z_{t}
$$

for all $t \geq 0$. Moreover, $\mathrm{E}\left[Z_{t}\right]=\mathrm{E}\left[Z_{0}\right]=1$. These equalities imply that we can define the probability measure $\mathbb{P}^{\theta, V}$ such that

$$
\mathbb{P}^{\theta, V}(A)=\mathrm{E}\left[\mathbb{1}_{A} Z_{T}\right], \quad A \in \mathcal{F}_{T}^{R, F} .
$$


Let $\sqrt{-1}$ be an imaginary unit. For any fixed $u \in \mathbb{R}^{K}$, by the Bayes rule, it holds that

$$
\begin{aligned}
\mathrm{E}^{\theta, V}\left[\exp \left\{\sqrt{-1} u^{\prime} F_{t+1}\right\} \mid \mathcal{F}_{t}^{R, F}\right] & =\mathrm{E}\left[\exp \left\{\sqrt{-1} u^{\prime} F_{t+1}\right\} \zeta_{t+1} \mid \mathcal{F}_{t}^{R, F}\right] \\
& =\mathrm{E}\left[\exp \left\{\sqrt{-1} u^{\prime} F_{t+1}\right\} \mathrm{E}\left[\zeta_{t+1} \mid \mathcal{F}_{t}^{R, F}, F_{t+1}\right] \mid \mathcal{F}_{t}^{R, F}\right] \\
& =\mathrm{E}\left[\exp \left\{\sqrt{-1} u^{\prime} F_{t+1}\right\} \mid \mathcal{F}_{t}^{R, F}\right] .
\end{aligned}
$$

This implies that the conditional distribution of $F_{t+1}$ given $\mathcal{F}_{t}^{R, F}$ under $\mathbb{P}^{\theta, V}$ is the same as the conditional distribution of $F_{t+1}$ given $\mathcal{F}_{t}^{R, F}$ under $\mathbb{P}$. It follows that

$$
\begin{aligned}
\mathrm{E}^{\theta, V}\left[R_{t+1} \mid \mathcal{F}_{t}^{R, F}\right] & =\mathrm{E}^{\theta, V}\left[\mathrm{E}^{\theta, V}\left[R_{t+1} \mid \mathcal{F}_{t}^{R, F}, F_{t+1}\right] \mid \mathcal{F}_{t}^{R, F}\right] \\
& =\mathrm{E}^{\theta, V}\left[\mu\left(F_{t+1}\right) \mid \mathcal{F}_{t}^{R, F}\right]+\theta_{t}=\mathrm{E}\left[\mu\left(F_{t+1}\right) \mid \mathcal{F}_{t}^{R, F}\right]+\theta_{t} \\
& =\mathrm{E}\left[R_{t+1} \mid \mathcal{F}_{t}^{R, F}\right]+\theta_{t},
\end{aligned}
$$

for all $t \geq 0$. Similarly, it holds that

$$
\begin{aligned}
\operatorname{Var}^{\theta, V}\left(R_{t+1} \mid \mathcal{F}_{t}^{R, F}\right)= & \operatorname{Var}^{\theta, V}\left(\mathrm{E}^{\theta, V}\left[R_{t+1} \mid \mathcal{F}_{t}^{R, F}, F_{t+1}\right] \mid \mathcal{F}_{t}^{R, F}\right) \\
& \quad+\mathrm{E}^{\theta, V}\left[\operatorname{Var}^{\theta, V}\left(R_{t+1} \mid \mathcal{F}_{t}^{R, F}, F_{t+1}\right) \mid \mathcal{F}_{t}^{R, F}\right] \\
= & \operatorname{Var}^{\theta, V}\left(\mu\left(F_{t+1}\right) \mid \mathcal{F}_{t}^{R, F}\right)+\mathrm{E}^{\theta, V}\left[\sigma\left(F_{t+1}\right)\left(\sigma\left(F_{t+1}\right)\right)^{\prime} \mid \mathcal{F}_{t}^{R, F}\right]+V_{t} \\
= & \operatorname{Var}\left(\mu\left(F_{t+1}\right) \mid \mathcal{F}_{t}^{R, F}\right)+\mathrm{E}\left[\sigma\left(F_{t+1}\right)\left(\sigma\left(F_{t+1}\right)\right)^{\prime} \mid \mathcal{F}_{t}^{R, F}\right]+V_{t} \\
= & \operatorname{Var}\left(R_{t+1} \mid \mathcal{F}_{t}^{R, F}\right)+V_{t},
\end{aligned}
$$

for all $t \geq 0$.

\section{A.2 Proofs of Theorem 5 and Proposition 6}

To prove Theorem 5, we need to show two lemmas. The objective function in the problem (2.2) can be written as

$$
\begin{aligned}
\mathrm{E}^{\theta, V}\left[\sum_{t=0}^{T-1} \delta^{t} X_{t} c_{t}\left(F_{t}, \theta_{t}, V_{t}, u_{t}\right)\right] & =\mathrm{E}\left[\sum_{t=0}^{T-1} \delta^{t} X_{t} c_{t}\left(F_{t}, \theta_{t}, V_{t}, u_{t}\right) Z_{t}\right] \\
& =\mathrm{E}\left[\sum_{t=0}^{T-1} \delta^{t} X_{t} c_{t}\left(F_{t}, \theta_{t}, V_{t}, u_{t}\right) \prod_{s=0}^{t} \zeta_{s}\right] .
\end{aligned}
$$

Let $\rho_{t}$ be a mapping such that

$$
\rho_{t}(W)=\delta \mathrm{E}\left[W \zeta_{t+1} \mid \mathcal{F}_{t}^{R, S}\right], \quad 0 \leq t \leq T-1 .
$$

Then, we can express the objective as

$$
\begin{aligned}
J_{T}(x, f, \theta, V, u):= & \mathrm{E}^{\theta, V}\left[\sum_{t=0}^{T-1} \delta^{t} X_{t} c_{t}\left(F_{t}, \theta_{t}, V_{t}, u_{t}\right)\right] \\
= & x c_{0}\left(f, \theta_{0}, V_{0}, u_{0}\right)+\rho_{0}\left(X_{1} c_{1}\left(F_{1}, \theta_{1}, V_{1}, u_{1}\right)+\rho_{1}(\cdots\right. \\
& \left.\left.+\rho_{T-2}\left(X_{T-1} c_{T-1}\left(F_{T-1}, \theta_{T-1}, V_{T-1}, u_{T-1}\right)\right) \cdots\right)\right),
\end{aligned}
$$


for all $u \in \mathcal{A}_{T}^{u}$ and $(\theta, V)=\left(\theta_{t}, V_{t}\right)_{t=0}^{T-1} \in \mathcal{A}_{T}^{\theta, V}$, where $X_{0}=x$ and $F_{0}=f$. The above recursive structure is important for the optimization. To simplify the notations, we write

$$
c_{t}^{u, \theta, V}=X_{t} c_{t}\left(F_{t}, \theta_{t}, V_{t}, u_{t}\right), \quad 0 \leq t \leq T-1 .
$$

We define $\rho_{t, r-1}$ as

$$
\rho_{t, r-1}\left(W_{t}, \ldots, W_{r}\right)=W_{t}+\rho_{t}\left(W_{t+1}+\rho_{t+1}\left(\cdots+\rho_{r-1}\left(W_{r}\right)\right)\right) .
$$

Then, for all $u \in \mathcal{A}_{T}^{u}$ and $(\theta, V) \in \mathcal{A}^{\theta, V}$, we have

$$
J_{T}(x, f, \theta, V, u)=\rho_{0, t-1}\left(c_{0}^{u, \theta, V}, \ldots, c_{t-1}^{u, \theta, V}, \rho_{t, T-2}\left(c_{t}^{u, \theta, V}, \ldots, c_{t-2}^{u, \theta, V}, c_{T-1}^{u, \theta, V}\right)\right),
$$

for all $0 \leq t \leq T-1$. We first prove that the order of the minimization with respect to $(\theta, V)$ can be exchangeable. The proof of the following lemma is essentially the same as the proof of Lemma 1 in Chen et al. (2014).

Lemma 11 Fix any $u \in \mathcal{A}_{T}^{u}$. Then, it holds that

$$
\begin{aligned}
& \min _{\left.\left(\theta_{r}, V_{r}\right)_{r=t}^{T-1} \in \mathcal{A}_{T}^{\theta, V}\right|_{t} ^{T-1}} \rho_{t, T-1}\left(c_{t}^{u, \theta, V}, \ldots, c_{T-1}^{u, \theta, V}\right) \\
& =\min _{\left.\left(\theta_{r}, V_{r}\right)_{r=t}^{s-1} \in \mathcal{A}_{T}^{\theta, V}\right|_{t} ^{s-1}} \rho_{t, s-1}\left(c_{0}^{u, \theta, V}, \ldots, c_{s-1}^{u, \theta, V}, \min _{\left.\left(\theta_{r}, V_{r}\right)_{r=t}^{s-1} \in \mathcal{A}_{T}^{\theta, V}\right|_{s} ^{T-1}} \rho_{s, T-2}\left(c_{s}^{u, \theta, V}, \ldots, c_{T-1}^{u, \theta, V}\right)\right),
\end{aligned}
$$

for all $0 \leq t \leq s \leq T-1$, where $\left.\mathcal{A}_{T}^{\theta, V}\right|_{t} ^{r}$ is a set of $\left(\theta_{s}, V_{s}\right)_{s=t}^{r}$ which are components of $(\theta, V) \in \mathcal{A}_{T}^{\theta, V}$ from the time $t$ to $r$.

Proof. Fix any $u \in \mathcal{A}_{T}^{u}, x \in \mathbb{R}$ and $f \in \mathbb{R}^{K}$. Fix any $0 \leq t \leq s \leq T-1$. We define $(\widetilde{\theta}, \widetilde{V})$ as

$$
(\widetilde{\theta}, \widetilde{V})=\left(\widetilde{\theta}_{r}, \widetilde{V}_{r}\right)_{r=s}^{T-1} \in \arg \min _{\left.\left(\theta_{r}, V_{r}\right)_{r=s}^{T-1} \in \mathcal{A}_{T}^{\theta, V}\right|_{s} ^{T-1}} \rho_{s, T-2}\left(c_{s}^{u, \theta, V}, \ldots, c_{T-2}^{u, \theta, V}, c_{T-1}^{u, \theta, V}\right) .
$$

Then, for every $(\theta, V) \in \mathcal{A}_{T}^{\theta, V}$, it holds that

$$
\rho_{s, T-2}\left(c_{s}^{u, \theta, V}, \ldots, c_{T-2}^{u, \theta, V}, c_{T-1}^{u, \theta, V}\right) \geq \rho_{s, T-2}\left(c_{s}^{u, \widetilde{\theta}, \widetilde{V}}, \ldots, c_{T-2}^{u, \widetilde{\theta}, \widetilde{V}}, c_{T-1}^{u, \widetilde{\theta}, \widetilde{V}}\right) .
$$

Since $\rho_{r}(W) \leq \rho_{r}\left(W^{\prime}\right)$ for all $r$ if $W \leq W^{\prime}$, it holds that

$$
\rho_{t, s-1}\left(W_{t}, \ldots, W_{s-1}, W_{s}\right) \leq \rho_{t, s-1}\left(W_{t}, \ldots, W_{s-1}, W^{\prime}\right),
$$

if $W_{s} \leq W^{\prime}$. Therefore, we have

$$
\rho_{t, T-1}\left(c_{t}^{u, \theta, V}, \ldots, c_{T-1}^{u, \theta, V}\right) \geq \rho_{t, s-1}\left(c_{t}^{u, \theta, V}, \ldots, c_{s-1}^{u, \theta, V}, \rho_{s, T-2}\left(c_{s}^{u, \widetilde{\theta}, \widetilde{V}}, \ldots, c_{T-2}^{u, \widetilde{\theta}, \widetilde{V}}, c_{T-1}^{u, \widetilde{\theta}, \widetilde{V}}\right)\right) .
$$

Minimizing the above inequality, we obtain

$$
\begin{aligned}
& \min _{\left.\left(\theta_{r}, V_{r}\right)_{r=t}^{T-1} \in \mathcal{A}_{T}^{\theta, V}\right|_{t} ^{T-1}} \rho_{t, T-1}\left(c_{t}^{u, \theta, V}, \ldots, c_{T-1}^{u, \theta, V}\right) \\
& \geq \min _{\left.\left(\theta_{r}, V_{r}\right)_{r=t}^{s-1} \in \mathcal{A}_{T}^{\theta, V}\right|_{t} ^{s-1}} \rho_{t, s-1}\left(c_{t}^{u, \theta, V}, \ldots, c_{s-1}^{u, \theta, V}, \rho_{s, T-2}\left(c_{s}^{u, \widetilde{\theta}, \widetilde{V}}, \ldots, c_{T-2}^{u, \widetilde{\theta}, \widetilde{V}}, c_{T-1}^{u, \widetilde{\theta}, \widetilde{V}}\right)\right) \\
& =\min _{\left.\left(\theta_{r}, V_{r}\right)_{r=t}^{s-1} \in \mathcal{A}_{T}^{\theta, V}\right|_{t} ^{s-1}} \rho_{t, s-1}\left(c_{t}^{u, \theta, V}, \ldots, c_{s-1}^{u, \theta, V}, \min _{\left.\left(\theta_{r}, V_{r}\right)_{r=s}^{T-1} \in \mathcal{A}_{T}^{\theta, V}\right|_{s} ^{T-1}} \rho_{s, T-2}\left(c_{s}^{u, \theta, V}, \ldots, c_{T-1}^{u, \theta, V}\right)\right) .
\end{aligned}
$$


We denote by $(\widehat{\theta}, \widehat{V})$ the following minimizer:

$$
(\widehat{\theta}, \widehat{V}):=\left(\widehat{\theta}_{r}, \widehat{V}_{r}\right)_{r=t}^{s-1} \in \arg \min _{\left.\left(\theta_{r}, V_{r}\right)_{r=t}^{s=1} \in \mathcal{A}_{T}^{\theta, V}\right|_{t} ^{s-1}} \rho_{t, s-1}\left(c_{t}^{u, \theta, V}, \ldots, c_{s-1}^{u, \theta, V}, \rho_{s, T-2}\left(c_{s}^{u, \widetilde{\theta}, \widetilde{V}}, \ldots, c_{T-1}^{u, \widetilde{\theta}}, \widetilde{V}\right)\right) .
$$

Then, $(\bar{\theta}, \bar{V}):=\left(\left(\widehat{\theta}_{t} \widehat{V}_{t}\right), \ldots,\left(\widehat{\theta}_{s-1}, \widehat{V}_{s-1}\right),\left(\widetilde{\theta}_{s}, \widetilde{V}_{s}\right), \ldots,\left(\widetilde{\theta}_{T-1}, \widetilde{V}_{T-1}\right)\right)$ is in $\left.\mathcal{A}_{T}^{\theta, V}\right|_{t} ^{T-1}$. It follows that

$$
\begin{aligned}
& \min _{\left.\left(\theta_{r}, V_{r}\right)_{r=t}^{T-1} \in \mathcal{A}_{T}^{\theta, V}\right|_{t} ^{T-1}} \rho_{t, T-1}\left(c_{t}^{u, \theta, V}, \ldots, c_{T-1}^{u, \theta, V}\right) \\
& \leq \rho_{t, T-1}\left(c_{t}^{u, \bar{\theta}, \bar{V}}, \ldots, c_{T-1}^{u, \bar{\theta}, \bar{V}}\right)=\rho_{t, s-1}\left(c_{t}^{u, \widehat{\theta}, \widehat{V}}, \ldots, c_{s-1}^{u, \widehat{\theta}, \widehat{V}}, \rho_{s, T-2}\left(c_{s}^{u, \widetilde{\theta}, \widetilde{V}}, \ldots, c_{T-2}^{u, \widetilde{\theta}, \widetilde{V}}, c_{T-1}^{u, \widetilde{\theta}, \widetilde{V}}\right)\right) \\
& =\min _{\left.\left(\theta_{r}, V_{r}\right)_{r=t}^{s-1} \in \mathcal{A}_{T}^{\theta, V}\right|_{t} ^{s-1}} \rho_{t, s-1}\left(c_{t}^{u, \theta, V}, \ldots, c_{s-1}^{u, \theta, V}, \min _{\left.\left(\theta_{r}, V_{r}\right)_{r=s}^{T-1} \in \mathcal{A}_{T}^{\theta, V}\right|_{s} ^{T-1}} \rho_{s, T-2}\left(c_{s}^{u, \theta, V}, \ldots, c_{T-1}^{u, \theta, V}\right)\right) .
\end{aligned}
$$

Therefore, we can conclude that the equality (A.2) holds.

By Lemma 11, we can minimize the objective function with respect to $(\theta, V)$, iteratively. Let

$$
\begin{aligned}
\bar{\rho}_{T-1}\left(u_{T-1}\right) & =\min _{\left.\left(\theta_{T-1}, V_{T-1}\right) \in \mathcal{A}_{T}^{\theta, V}\right|_{T-1} ^{T-1}} c_{T-1}^{u, \theta, V}, \\
\bar{\rho}_{t}\left(W, u_{t}\right) & =\min _{\left.\left(\theta_{t}, V_{t}\right) \in \mathcal{A}_{T}^{\theta, V}\right|_{t} ^{t}}\left\{c_{t}^{u, \theta, V}+\delta \mathrm{E}\left[W \zeta_{t+1} \mid \mathcal{F}_{t}^{R, F}\right]\right\}, \quad 0 \leq t \leq T-2, \\
\bar{\rho}_{t, s-1}\left(W,\left(u_{r}\right)_{r=t}^{s-1}\right) & =\bar{\rho}_{t}\left(\bar{\rho}_{t+1}\left(\cdots, \bar{\rho}_{s-1}\left(W, u_{s-1}\right) \cdots, u_{t+1}\right), u_{t}\right)
\end{aligned}
$$

Then, by Lemma 11, we derive that

$$
J_{T}^{*}(x, f, u):=\min _{(\theta, V) \in \mathcal{A}_{T}^{\theta, V}} J_{T}(x, f, \theta, V, u)=\bar{\rho}_{0, T-1}\left(\bar{\rho}_{T-1}\left(u_{T-1}\right),\left(u_{t}\right)_{t=0}^{T-2}\right),
$$

for all $u=\left(u_{t}\right)_{t=0}^{T-1} \in \mathcal{A}_{T}^{u}$.

The investor needs to maximize $J_{T}^{*}(x, f, u)$ over $u \in \mathcal{A}_{T}^{u}$. In the proof of Lemma 11, the monotonicity of $\rho_{t}$ plays a key role in the exchangeability of the minimization with respect to $(\theta, V)$. Similarly, the monotonicity of $\bar{\rho}_{t}$ is important to the the order of the maximization with respect to $u$. $\bar{\rho}_{t}$ satisfies the monotonicity, that is, for all $y \in \mathbb{R}^{d}$ and $0 \leq t \leq T-1$, $\bar{\rho}_{t}(W, y) \leq \bar{\rho}_{t}\left(W^{\prime}, y\right)$ holds if $W \leq W^{\prime}$. This implies that for any $u \in \mathcal{A}_{T}^{u}$,

$$
\bar{\rho}_{t, r-1}\left(W_{r},\left(u_{s}\right)_{s=t}^{r-1}\right) \leq \bar{\rho}_{t, r-1}\left(W^{\prime},\left(u_{s}\right)_{s=t}^{r-1}\right),
$$

if $W_{r} \leq W^{\prime}$. Therefore, using the same argument as in Lemma 11, we can prove the following lemma.

Lemma 12 For all $0 \leq t \leq s \leq T-1$, it holds that

$$
\begin{aligned}
& \max _{\left.\left(u_{r}\right)_{r=t}^{T-1} \in \mathcal{A}_{T}^{u}\right|_{t} ^{T-1}} \bar{\rho}_{t, T-1}\left(\bar{\rho}_{T-1}\left(u_{T-1}\right),\left(u_{r}\right)_{r=t}^{T-2}\right) \\
& =\max _{\left.\left(u_{r}\right)_{r=t}^{s-1} \in \mathcal{A}_{T}^{u}\right|_{t} ^{s-1}} \bar{\rho}_{t, s-1}\left(\max _{\left.\left(u_{r}\right)_{r=s}^{T-1} \in \mathcal{A}_{T}^{u}\right|_{s} ^{T-1}} \bar{\rho}_{s, T-2}\left(\rho_{T-1}\left(u_{T-1}\right),\left(u_{r}\right)_{r=s}^{T-2}\right),\left(u_{r}\right)_{r=t}^{s-1}\right),
\end{aligned}
$$

where $\left.\mathcal{A}_{T}^{u}\right|_{t} ^{r}$ is a set of $\left(u_{s}\right)_{s=t}^{r}$ which are components of $u \in \mathcal{A}_{T}^{u}$ from the time $t$ to $r$. 
Using Lemma 12, the dynamic programming of the multiple priors optimization can be justified. Now, we prove Theorem 5 and Proposition 6.

Proof of Theorem 5 and Proposition 6. Consider the time $T-1$ problem

$$
\begin{aligned}
& \max _{\left.u_{T-1} \in \mathcal{A}_{T}^{u}\right|_{T-1} ^{T-1}} \bar{\rho}_{T-1}\left(u_{T-1}\right) \\
& =\max _{\left.\left.u_{T-1} \in \mathcal{A}_{T}^{u}\right|_{T-1} ^{T-1}\left(\theta_{T-1}, V_{T-1}\right) \in \mathcal{A}_{T}^{\theta, V}\right|_{T-1} ^{T-1}} c_{T-1}^{u, \theta, V} \\
& =\max _{\left.u_{T-1} \in \mathcal{A}_{T}^{u}\right|_{T-1} ^{T-1}} \min _{\left.\left(\theta_{T-1}, V_{T-1}\right) \in \mathcal{A}_{T}^{\theta, V}\right|_{T-1} ^{T-1}} X_{T-1} c_{T-1}\left(F_{T-1}, \theta_{T-1}, V_{T-1}, u_{T-1}\right) \\
& =\max _{\left.\left.u_{T-1} \in \mathcal{A}_{T}^{u}\right|_{T-1} ^{T-1}\left(\theta_{T-1}, V_{T-1}\right) \in \mathcal{A}_{T}^{\theta, V}\right|_{T-1} ^{T-1}} X_{T-1}\left\{1+r_{\mathrm{f}}+\left(m^{e}\left(F_{T-1}\right)+\theta_{T-1}\right)^{\prime} u_{T-1}\right. \\
& \left.-\frac{\widehat{\gamma}_{T-1}}{2} u_{T-1}^{\prime}\left(A\left(F_{T-1}\right)+V_{T-1}\right) u_{T-1}\right\} .
\end{aligned}
$$

Then, the minimization problem in the problem (A.3) is reduced to the following problem,

$$
\min _{\left.\left(\theta_{T-1}, V_{T-1}\right) \in \mathcal{A}_{T}^{\theta, V}\right|_{T-1} ^{T-1}}\left\{\theta_{T-1}^{\prime} u_{T-1}-\frac{\widehat{\gamma}_{T-1}}{2} u_{T-1}^{\prime} V_{T-1} u_{T-1}\right\} .
$$

The Lagrange function of the problem (A.4) is

$$
\begin{aligned}
\mathcal{L}^{\theta, V}= & \theta_{T-1}^{\prime} u_{T-1}-\frac{\widehat{\gamma}_{T-1}}{2} u_{T-1}^{\prime} V_{T-1} u_{T-1}-\lambda_{\theta}\left(\left(\eta_{T-1}^{\theta}\right)^{2}-\theta_{T-1}^{\prime}\left(A\left(F_{T-1}\right)+V_{T-1}\right)^{-1} \theta_{T-1}\right) \\
& -\lambda_{V}\left(\left(\eta_{T-1}^{V}\right)^{2}\left\|A\left(F_{T-1}\right)\right\|^{2}-\left\|V_{T-1}\right\|^{2}\right),
\end{aligned}
$$

where $\lambda_{\theta}$ and $\lambda_{V}$ are Lagrange multipliers. We first consider the case when $u_{T-1} \neq \mathbf{0}_{d}$. The first order condition for $\theta_{T-1}$ is

$$
u_{T-1}+2 \lambda_{\theta}\left(A\left(F_{T-1}\right)+V_{T-1}\right)^{-1} \theta_{T-1}=\mathbf{0}_{d} .
$$

Hence, we have

$$
\theta_{T-1}=-\frac{1}{2 \lambda_{\theta}}\left(A\left(F_{T-1}\right)+V_{T-1}\right) u_{T-1} .
$$

Since the constraint for $\theta_{T-1}$ is binded, it holds that

$$
\left(\eta_{T-1}^{\theta}\right)^{2}=\theta_{T-1}^{\prime}\left(A\left(F_{T-1}\right)+V_{T-1}\right)^{-1} \theta_{T-1}=\frac{1}{4 \lambda_{\theta}^{2}} u_{T-1}^{\prime}\left(A\left(F_{T-1}\right)+V_{T-1}\right) u_{T-1} .
$$

Hence,

$$
\lambda_{\theta}=\frac{1}{2 \eta_{T-1}^{\theta}} \sqrt{u_{T-1}^{\prime}\left(A\left(F_{T-1}\right)+V_{T-1}\right) u_{T-1}},
$$

and

$$
\theta_{T-1}=-\frac{\eta_{T-1}^{\theta}}{\sqrt{u_{T-1}^{\prime}\left(A\left(F_{T-1}\right)+V_{T-1}\right) u_{T-1}}}\left(A\left(F_{T-1}\right)+V_{T-1}\right) u_{T-1} .
$$

We denote the $i \times j$ th element of $V_{T-1}$ by $v_{T-1}^{i, j}$. Then, the first order condition for $v_{T-1}^{i, j}$ is

$$
\begin{aligned}
& -\widehat{\gamma}_{T-1} u_{T-1}^{i} u_{T-1}^{j}+4 \lambda_{V} v_{T-1}^{i, j} \\
& \quad=\operatorname{tr}\left\{\left(A\left(F_{T-1}\right)+V_{T-1}\right)^{-1} \theta_{T-1} \theta_{T-1}^{\prime}\left(A\left(F_{T-1}\right)+V_{T-1}\right)^{-1} N^{i, j}\right\}, \text { if } i \neq j, \\
& -\frac{\widehat{\gamma}_{T-1}}{2}\left(u_{T-1}^{i}\right)^{2}+2 \lambda_{V} v_{T-1}^{i, j} \\
& \quad=\operatorname{tr}\left\{\left(A\left(F_{T-1}\right)+V_{T-1}\right)^{-1} \theta_{T-1} \theta_{T-1}^{\prime}\left(A\left(F_{T-1}\right)+V_{T-1}\right)^{-1} N^{i, i}\right\}, \text { if } i=j,
\end{aligned}
$$


where $N^{i, j}$ is a $d$-dimensional matrix whose $i \times j$ th element and $j \times i$ th element are 1 and the other elements are zero. Substituting $\theta_{T-1}$ into $\left(A\left(F_{T-1}\right)+V_{T-1}\right)^{-1} \theta_{T-1} \theta_{T-1}^{\prime}\left(A\left(F_{T-1}\right)+V_{T-1}\right)^{-1}$, we have

$$
\begin{aligned}
& \left(A\left(F_{T-1}\right)+V_{T-1}\right)^{-1} \theta_{T-1} \theta_{T-1}^{\prime}\left(A\left(F_{T-1}\right)+V_{T-1}\right)^{-1} \\
& =\frac{\left(\eta_{T-1}^{\theta}\right)^{2}}{u_{T-1}^{\prime}\left(A\left(F_{T-1}\right)+V_{T-1}\right) u_{T-1}} u_{T-1} u_{T-1}^{\prime}
\end{aligned}
$$

Hence, it holds that

$$
\begin{aligned}
& \operatorname{tr}\left\{\left(A\left(F_{T-1}\right)+V_{T-1}\right)^{-1} \theta_{T-1} \theta_{T-1}^{\prime}\left(A\left(F_{T-1}\right)+V_{T-1}\right)^{-1} N^{i, j}\right\} \\
& = \begin{cases}\frac{2\left(\eta_{T-1}^{\theta}\right)^{2}}{u_{T-1}^{\prime}\left(A\left(F_{T-1}\right)+V_{T-1}\right) u_{T-1}} u_{T-1}^{i} u_{T-1}^{j}, & \text { if } i \neq j \\
\frac{\left(\eta_{T-1}^{\theta}\right)^{2}}{u_{T-1}^{\prime}\left(A\left(F_{T-1}\right)+V_{T-1}\right) u_{T-1}}\left(u_{T-1}^{i}\right)^{2}, & \text { if } i=j\end{cases}
\end{aligned}
$$

It follows that

$$
-\widehat{\gamma}_{T-1} u_{T-1}^{i} u_{T-1}^{j}+4 \lambda_{V} v_{T-1}^{i, j}=\frac{2\left(\eta_{T-1}^{\theta}\right)^{2}}{u_{T-1}^{\prime}\left(A\left(F_{T-1}\right)+V_{T-1}\right) u_{T-1}} u_{T-1}^{i} u_{T-1}^{j},
$$

for all $i$ and $j$. Using the matrix notation, we have

$$
-\widehat{\gamma}_{T-1} u_{T-1} u_{T-1}^{\prime}+4 \lambda_{V} V_{T-1}=\frac{2\left(\eta_{T-1}^{\theta}\right)^{2}}{u_{T-1}^{\prime}\left(A\left(F_{T-1}\right)+V_{T-1}\right) u_{T-1}} u_{T-1} u_{T-1}^{\prime} .
$$

Hence,

$$
V_{T-1}=\frac{1}{4 \lambda_{V}}\left(\widehat{\gamma}_{T-1}+\frac{2\left(\eta_{T-1}^{\theta}\right)^{2}}{u_{T-1}^{\prime}\left(A\left(F_{T-1}\right)+V_{T-1}\right) u_{T-1}}\right) u_{T-1} u_{T-1}^{\prime} .
$$

Since the constraint for $V_{T-1}$ is also binded, it holds that

$$
\begin{aligned}
& \left(\eta_{T-1}^{V}\right)^{2}\left\|A\left(F_{T-1}\right)\right\|^{2} \\
& =\left\|V_{T-1}\right\|^{2}=\frac{1}{16 \lambda_{V}^{2}}\left(\widehat{\gamma}_{T-1}+\frac{2\left(\eta_{T-1}^{\theta}\right)^{2}}{u_{T-1}^{\prime}\left(A\left(F_{T-1}\right)+V_{T-1}\right) u_{T-1}}\right)^{2}\left\|u_{T-1}\right\|^{4} .
\end{aligned}
$$

Therefore, the Lagrange multiplier $\lambda_{V}$ is

$$
\lambda_{V}=\frac{1}{4 \eta_{T-1}^{V}\left\|A\left(F_{T-1}\right)\right\|}\left(\widehat{\gamma}_{T-1}+\frac{2\left(\eta_{T-1}^{\theta}\right)^{2}}{u_{T-1}^{\prime}\left(A\left(F_{T-1}\right)+V_{T-1}\right) u_{T-1}}\right)\left\|u_{T-1}\right\|^{2} .
$$

Hence, we have

$$
V_{T-1}=\frac{\eta_{T-1}^{V}\left\|A\left(F_{T-1}\right)\right\|}{\left\|u_{T-1}\right\|^{2}} u_{T-1} u_{T-1}^{\prime}
$$

Then,

$$
\left(A\left(F_{T-1}\right)+V_{T-1}\right) u_{T-1}=\left(A\left(F_{T-1}\right)+\eta_{T-1}^{V}\left\|A\left(F_{T-1}\right)\right\| I_{d}\right) u_{T-1}
$$

and

$$
\theta_{T-1}=-\frac{\eta_{T-1}^{\theta}\left(A\left(F_{T-1}\right)+\eta_{T-1}^{V}\left\|A\left(F_{T-1}\right)\right\| I_{d}\right) u_{T-1}}{\sqrt{u_{T-1}^{\prime}\left(A\left(F_{T-1}\right)+\eta_{T-1}^{V}\left\|A\left(F_{T-1}\right)\right\| I_{d}\right) u_{T-1}}} .
$$

If $u_{T-1}=\mathbf{0}_{d}$, then every $\left.\left(\theta_{T-1}, V_{T-1}\right) \in \mathcal{A}_{T}^{\theta, V}\right|_{T-1} ^{T-1}$ is a solution. In this case, we choose $\theta_{T-1}=\mathbf{0}_{d}$ and $V_{T-1}=\mathbf{O}_{d}$ as the solution. In all cases, $\left(\theta_{T-1}, V_{T-1}\right)$ is in $\left.\mathcal{A}_{T}^{\theta, V}\right|_{T-1} ^{T-1}$. 
Substituting $\theta_{T-1}$ and $V_{T-1}$ into the original time $T-1$ optimization problem (A.3), we obtain

$$
\begin{aligned}
\max _{u_{T-1} \in \mathcal{A}_{T}^{u} T_{T-1}^{T-1}} X_{T-1}\{ & 1+r_{\mathrm{f}}+\left(m^{e}\left(F_{T-1}\right)\right)^{\prime} u_{T-1} \\
& -\frac{\widehat{\gamma}_{T-1}}{2} u_{T-1}^{\prime}\left(A\left(F_{T-1}\right)+\eta_{T-1}^{V}\left\|A\left(F_{T-1}\right)\right\| I_{d}\right) u_{T-1} \\
& \left.-\eta_{T-1}^{\theta} \sqrt{u_{T-1}^{\prime}\left(A\left(F_{T-1}\right)+\eta_{T-1}^{V}\left\|A\left(F_{T-1}\right)\right\| I_{d}\right) u_{T-1}}\right\} .
\end{aligned}
$$

We first consider the following case.

$$
a_{T-1}^{e}:=\left(m^{e}\left(F_{T-1}\right)\right)^{\prime}\left(A\left(F_{T-1}\right)+\eta_{T-1}^{V}\left\|A\left(F_{T-1}\right)\right\| I_{d}\right)^{-1} m^{e}\left(F_{T-1}\right)>\left(\eta_{T-1}^{\theta}\right)^{2} .
$$

The first order condition of this problem (A.5) is

$$
m^{e}\left(F_{T-1}\right)-\left(\widehat{\gamma}_{T-1}+\frac{\eta_{T-1}^{\theta}}{\psi_{T-1}}\right)\left(A\left(F_{T-1}\right)+\eta_{T-1}^{V}\left\|A\left(F_{T-1}\right)\right\| I_{d}\right) u_{T-1}=\mathbf{0}_{d},
$$

where

$$
\psi_{T-1}=\sqrt{u_{T-1}^{\prime}\left(A\left(F_{T-1}\right)+\eta_{T-1}^{V}\left\|A\left(F_{T-1}\right)\right\| I_{d}\right) u_{T-1}}
$$

Hence, we have

$$
u_{T-1}=\left(\widehat{\gamma}_{T-1}+\frac{\eta_{T-1}^{\theta}}{\psi_{T-1}}\right)^{-1}\left(A\left(F_{T-1}\right)+\eta_{T-1}^{V}\left\|A\left(F_{T-1}\right)\right\| I_{d}\right)^{-1} m^{e}\left(F_{T-1}\right) .
$$

Furthermore, we also have

$$
\begin{aligned}
\psi_{T-1}^{2} & =u_{T-1}^{\prime}\left(A\left(F_{T-1}\right)+\eta_{T-1}^{V}\left\|A\left(F_{T-1}\right)\right\| I_{d}\right) u_{T-1} \\
& =\left(\widehat{\gamma}_{T-1}+\frac{\eta_{T-1}^{\theta}}{\psi_{T-1}}\right)^{-2} a_{T-1}^{e} .
\end{aligned}
$$

Thus, we obtain the following quadratic equation for $\psi_{T-1}$.

$$
\widehat{\gamma}_{T-1}^{2} \psi_{T-1}^{2}+2 \widehat{\gamma}_{T-1} \eta_{T-1}^{\theta} \psi_{T-1}+\left(\eta_{T-1}^{\theta}\right)^{2}-a_{T-1}^{e}=0 .
$$

By the inequality (A.6), the above equation has a unique positive solution such that

$$
\psi_{T-1}=\frac{\sqrt{a_{T-1}^{e}}-\eta_{T-1}^{\theta}}{\widehat{\gamma}_{T-1}} .
$$

Hence, the optimal portfolio at time $T-1$ is

$$
u_{T-1}=\frac{1}{\widehat{\gamma}_{T-1}}\left(1-\frac{\eta_{T-1}^{\theta}}{\sqrt{a_{T-1}^{e}}}\right)\left(A\left(F_{T-1}\right)+\eta_{T-1}^{V}\left\|A\left(F_{T-1}\right)\right\| I_{d}\right)^{-1} m^{e}\left(F_{T-1}\right) .
$$

Let $K_{T-1}=A\left(F_{T-1}\right)+\eta_{T-1}^{V}\left\|A\left(F_{T-1}\right)\right\| I_{d}$. If the inequality (A.6) does not hold, then the Cauchy-Schwartz inequality leads to

$$
\begin{aligned}
\eta_{T-1}^{\theta} \sqrt{u_{T-1}^{\prime} K_{T-1} u_{T-1}} & \geq \sqrt{a_{T-1}^{e}} \sqrt{u_{T-1}^{\prime} K_{T-1} u_{T-1}} \\
& =\sqrt{\left(m^{e}\left(F_{T-1}\right)\right)^{\prime} K_{T-1}^{-1} m^{e}\left(F_{T-1}\right)} \sqrt{u_{T-1}^{\prime} K_{T-1}\left(K_{T-1}\right)^{-1} K_{T-1} u_{T-1}} \\
& \geq\left|\left(m^{e}\left(F_{T-1}\right)\right)^{\prime}\left(K_{T-1}\right)^{-1} K_{T-1} u_{T-1}\right|=\left|\left(m^{e}\left(F_{T-1}\right)\right)^{\prime} u_{T-1}\right| .
\end{aligned}
$$


Hence,

$$
\begin{aligned}
& \max _{\left.u_{T-1} \in \mathcal{A}_{T}^{u}\right|_{T-1} ^{T-1}} X_{T-1}\{1+r_{\mathrm{f}}+\left(m^{e}\left(F_{T-1}\right)\right)^{\prime} u_{T-1} \\
&-\frac{\widehat{\gamma}_{T-1}}{2} u_{T-1}^{\prime}\left(A\left(F_{T-1}\right)+\eta_{T-1}^{V}\left\|A\left(F_{T-1}\right)\right\| I_{d}\right) u_{T-1} \\
&-\left.\eta_{T-1}^{\theta} \sqrt{u_{T-1}^{\prime}\left(A\left(F_{T-1}\right)+\eta_{T-1}^{V}\left\|A\left(F_{T-1}\right)\right\| I_{d}\right) u_{T-1}}\right\} \\
& \leq \max _{\left.u_{T-1} \in \mathcal{A}_{T}^{u}\right|_{T-1} ^{T-1}} X_{T-1}\left\{1+r_{\mathrm{f}}+\left(m^{e}\left(F_{T-1}\right)\right)^{\prime} u_{T-1}-\left|\left(m^{e}\left(F_{T-1}\right)\right)^{\prime} u_{T-1}\right|\right\}
\end{aligned}
$$

The solution of the later problem is an arbitrarily vector $\left.u_{T-1} \in \mathcal{A}_{T}^{u}\right|_{T-1} ^{T-1}$, such that $\left(m^{e}\left(F_{T-1}\right)\right)^{\prime} u_{T-1} \geq$ 0 . We choose $u_{T-1}=\mathbf{0}_{d}$, and then the optimal value of the later problem becomes $X_{T-1}(1+$ $\left.r_{\mathrm{f}}\right)$. Moreover, we have

$$
\begin{aligned}
& 1+r_{\mathrm{f}}+\left(m^{e}\left(F_{T-1}\right)\right)^{\prime} u_{T-1}-\frac{\widehat{\gamma}_{T-1}}{2} u_{T-1}^{\prime}\left(A\left(F_{T-1}\right)+\eta_{T-1}^{V}\left\|A\left(F_{T-1}\right)\right\| I_{d}\right) u_{T-1} \\
& -\eta_{T-1}^{\theta} \sqrt{u_{T-1}^{\prime}\left(A\left(F_{T-1}\right)+\eta_{T-1}^{V}\left\|A\left(F_{T-1}\right)\right\| I_{d}\right) u_{T-1}} \\
& =1+r_{\mathrm{f}},
\end{aligned}
$$

if $u_{T-1}=\mathbf{0}_{d}$. Therefore, $u_{T-1}=\mathbf{0}_{d}$ is also a solution of the former problem.

In each case, we can express the optimal portfolio at time $T-1$ as

$$
u_{T-1}=\frac{1}{\widehat{\gamma}_{T-1}}\left(1-\frac{\eta_{T-1}^{\theta}}{\sqrt{a_{T-1}^{e}}}\right)^{+}\left(A\left(F_{T-1}\right)+\eta_{T-1}^{V}\left\|A\left(F_{T-1}\right)\right\| I_{d}\right)^{-1} m^{e}\left(F_{T-1}\right) .
$$

It is clear that $\left.u_{T-1} \in \mathcal{A}_{T}^{u}\right|_{T-1} ^{T-1}$. In addition, the value function is

$$
\begin{aligned}
\max _{\left.u_{T-1} \in \mathcal{A}_{T}^{u}\right|_{T} ^{T}} \bar{\rho}_{T-1}\left(u_{T-1}\right) & =Y_{T-1}^{T} X_{T-1} \\
Y_{T-1}^{T} & =\max _{u_{T-1} \in \mathbb{R}^{d}} \min _{\left.\left(\theta_{T-1}, V_{T-1}\right) \in \mathcal{A}_{T}^{\theta, V}\right|_{T-1} ^{T-1}}\left\{c_{T-1}\left(F_{T-1}, \theta_{T-1}, V_{T-1}, u_{T-1}\right)\right\} \\
& =1+r_{\mathrm{f}}+\frac{\left(\left(\sqrt{a_{T-1}^{e}}-\eta_{T-1}^{\theta}\right)^{+}\right)^{2}}{2 \widehat{\gamma}_{T-1}} .
\end{aligned}
$$

By the definition, $Y_{T-1}^{T}$ is $\mathcal{F}_{T-1}^{F}$-measurable.

Now, we assume that

$$
\max _{\left.\left(u_{r}\right)_{r=t+1}^{T-1} \in \mathcal{A}_{T}^{u}\right|_{t+1} ^{T-1}} \underset{\left.\left(\theta_{r}, V_{r}\right)_{r=t+1}^{T-1} \in \mathcal{A}_{T}^{\theta, V}\right|_{t+1} ^{T-1}}{ } \rho_{t+1, T-1}\left(c_{t+1}^{u, \theta, V}, \ldots, c_{T-1}^{u, \theta, V}\right)=Y_{t+1}^{T} X_{t+1},
$$

for some $0 \leq t \leq T-2$. Then, we have

$$
\begin{aligned}
& \mathrm{E}^{\theta, V}\left[Y_{t+1}^{T} X_{t+1} \mid \mathcal{F}_{t}^{R, F}\right] \\
& =\mathrm{E}^{\theta, V}\left[Y_{t+1}^{T}\left(1+r_{\mathrm{f}}+\left(R_{t+1}^{e}\right)^{\prime} u_{t}\right) X_{t} \mid \mathcal{F}_{t}^{R, F}\right] \\
& =\left(1+r_{\mathrm{f}}\right) \mathrm{E}^{\theta, V}\left[Y_{t+1}^{T} \mid \mathcal{F}_{t}^{R, F}\right] X_{t}+\left(\mathrm{E}^{\theta, V}\left[Y_{t+1}^{T} R_{t+1} \mid \mathcal{F}_{t}^{R, F}\right]\right)^{\prime} u_{t} X_{t} \\
& =\left(1+r_{\mathrm{f}}\right) \mathrm{E}\left[Y_{t+1}^{T} \mid \mathcal{F}_{t}^{R, F}\right] X_{t}+\left(\mathrm{E}\left[Y_{t+1}^{T} \mu^{e}\left(F_{t+1}\right) \mid \mathcal{F}_{t}^{R, F}\right]+\mathrm{E}\left[Y_{t+1}^{T} \mid \mathcal{F}_{t}^{R, F}\right] \theta_{t}\right)^{\prime} u_{t} X_{t}
\end{aligned}
$$


where we have used Lemma 4 and $\mathcal{F}_{t+1}^{F}$-measurability of $Y_{t+1}^{T}$. Therefore, the objective in the time $t$ problem is

$$
c_{t}^{u, \theta, V}+\delta \mathrm{E}^{\theta, V}\left[Y_{t+1}^{T} X_{t+1} \mid \mathcal{F}_{t}^{R, F}\right]=X_{t}\left\{\left(1+r_{\mathrm{f}}\right) g_{t}^{e}+\left(m_{t}^{e}+g_{t}^{e} \theta_{t}\right)^{\prime} u_{t}-\frac{\widehat{\gamma}_{t}}{2} u_{t}^{\prime}\left(A_{t}+V_{t}\right) u_{t}\right\} .
$$

Hence, the objective function in the time $t$ problem has the same form as the objective function in the problem (A.3). Therefore, the error of the conditional mean is

$$
\theta_{t}=-\frac{\operatorname{sgn}\left(g_{t}^{e}\right) \eta_{t}^{\theta}\left(A\left(F_{t}\right)+\eta_{t}^{V}\left\|A\left(F_{t}\right)\right\| I_{d}\right) u_{t}}{\sqrt{u_{t}^{\prime}\left(A\left(F_{t}\right)+\eta_{t}^{V}\left\|A\left(F_{t}\right)\right\| I_{d}\right) u_{t}}}
$$

where sgn is a sign function such that

$$
\operatorname{sgn}(x)= \begin{cases}1, & \text { if } x>0 \\ -1, & \text { if } x<0 \\ 0, & \text { if } x=0 .\end{cases}
$$

The error of the conditional variance is

$$
V_{t}=\frac{\eta_{t}^{V}\left\|A\left(F_{t}\right)\right\|}{\left\|u_{t}\right\|^{2}} u_{t} u_{t}^{\prime} .
$$

The optimal portfolio at time $t$ is

$$
u_{t}=\frac{1}{\widehat{\gamma}_{t}}\left(1-\frac{\left|g_{t}^{e}\right| \eta_{t}^{\theta}}{\sqrt{a_{t}^{e}}}\right)^{+}\left(A\left(F_{t}\right)+\eta_{t}^{V}\left\|A\left(F_{t}\right)\right\| I_{d}\right)^{-1} m_{t}^{e}
$$

It is clear that $\left.u_{t} \in \mathcal{A}_{T}^{u}\right|_{t} ^{t}$ and $\left.\left(\theta_{t}, V_{t}\right) \in \mathcal{A}_{T}^{\theta, V}\right|_{t} ^{t}$. The value function is

$$
\begin{aligned}
Y_{t}^{T} X_{t} & =\max _{\left.\left(u_{r}\right)_{r=t}^{T-1} \in \mathcal{A}_{T}^{u}\right|_{t} ^{T-1}} \min _{\left.\left(\theta_{r}, V_{r}\right)_{r=t}^{T-1} \in \mathcal{A}_{T}^{\theta, V}\right|_{t} ^{T-1}} \rho_{t, T-1}\left(c_{t}^{u, \theta, V}, \ldots, c_{T-1}^{u, \theta, V}\right), \\
Y_{t}^{T} & =\left(1+r_{\mathrm{f}}\right) g_{t}^{e}+\frac{\left(\left(\sqrt{a_{t}^{e}}-\left|g_{t}^{e}\right| \eta_{t}^{\theta}\right)^{+}\right)^{2}}{2 \widehat{\gamma}_{t}} .
\end{aligned}
$$

By construction, $Y_{t}^{T}$ is $\mathcal{F}_{t}^{F}$-measurable.

For any $0 \leq t \leq T-1$, by the mathematical induction, the value function at time $t$ can be expressed as

$$
Y_{t}^{T} X_{t}=\max _{\left.\left(u_{r}\right)_{r=t}^{T-1} \in \mathcal{A}_{T}^{u}\right|_{t} ^{T-1}} \min _{\left.\left(\theta_{r}, V_{r}\right)_{r=t}^{T-1} \in \mathcal{A}_{T}^{\theta, V}\right|_{t} ^{T-1}} \rho_{t, T-1}\left(c_{t}^{u, \theta, V}, \ldots, c_{T-1}^{u, \theta, V}\right),
$$

where

$$
Y_{t}^{T}=\left(1+r_{\mathrm{f}}\right) g_{t}^{e}+\frac{\left(\left(\sqrt{a_{t}^{e}}-\left|g_{t}^{e}\right| \eta_{t}^{\theta}\right)^{+}\right)^{2}}{2 \widehat{\gamma}_{t}} .
$$

The optimal portfolio at time $t$ is

$$
u_{t}^{*}=\frac{1}{\widehat{\gamma}_{t}}\left(1-\frac{\left|g_{t}^{e}\right| \eta_{t}^{\theta}}{\sqrt{a_{t}^{e}}}\right)^{+}\left(A\left(F_{t}\right)+\eta_{t}^{V}\left\|A\left(F_{t}\right)\right\| I_{d}\right)^{-1} m_{t}^{e} .
$$

Also, it is obvious that the optimal $\left(u_{t}^{*}\right)_{t=0}^{T-1}$ is time-consistent. 


\section{A.3 Proofs of Proposition 7 and 8}

Proof of Proposition 7. By Theorem 5, we can use the following dynamic programming procedure.

$$
\begin{aligned}
\widehat{Y}_{T-1}^{T} & =\max _{\left.\left.u_{T-1} \in \widetilde{\mathcal{A}}_{T}^{u}\right|_{T-1} ^{T-1}\left(\theta_{T-1}, V_{T-1}\right) \in \mathcal{A}_{T}^{\theta, V}\right|_{T-1} ^{T-1}}\left\{\widehat{c}_{T-1}\left(F_{T-1}, \theta_{T-1}, V_{T-1}, u_{T-1}\right)\right\}, \\
\widehat{Y}_{t}^{T} & =\max _{\left.\left.u_{t} \in \widetilde{\mathcal{A}}_{T}^{u}\right|_{t} ^{t}\left(\theta_{t}, V_{t}\right) \mathcal{A}_{T}^{\theta, V}\right|_{t} ^{t}}\left\{\widehat{c}_{t}\left(F_{t}, \theta_{t}, V_{t}, u_{t}\right)+\delta \mathrm{E}^{\theta, V}\left[Y_{t+1}^{T}\left(1+\left(R_{t+1}\right)^{\prime} u_{t}\right) \mid \mathcal{F}_{t}^{R, S}\right]\right\} .
\end{aligned}
$$

Consider the time $T-1$ problem.

$$
\begin{aligned}
\widehat{Y}_{T-1}^{T}=\max _{\left.u_{T-1} \in \widetilde{\mathcal{A}}_{T}^{u} T_{T-1}^{T-1}\left(\theta_{T-1}, V_{T-1}\right) \in \mathcal{A}_{T}^{\theta, V}\right|_{T-1} ^{T-1}}\left\{1+\left(m\left(F_{T-1}\right)+\theta_{T-1}\right)^{\prime} u_{T-1}\right. \\
\left.-\frac{\widehat{\gamma}_{T-1}}{2} u_{T-1}^{\prime}\left(A\left(F_{T-1}\right)+V_{T-1}\right) u_{T-1}\right\} .
\end{aligned}
$$

The inner minimization problem in the problem (A.7) can be reduced to the following:

$$
\min _{\left.\left(\theta_{T-1}, V_{T-1}\right) \in \mathcal{A}_{T}^{\theta, V}\right|_{T-1} ^{T-1}}\left\{\theta_{T-1}^{\prime} u_{T-1}-\frac{\widehat{\gamma}_{T-1}}{2} u_{T-1}^{\prime} V_{T-1} u_{T-1}\right\} .
$$

The above problem is the same as the problem (A.4) in the proof of Theorem 5. Therefore, the solutions are

$$
\begin{aligned}
\theta_{T-1} & =-\frac{\eta_{T-1}^{\theta}\left(A\left(F_{T-1}\right)+\eta_{T-1}^{V}\left\|A\left(F_{T-1}\right)\right\| I_{d}\right) u_{T-1}}{\sqrt{u_{T-1}^{\prime}\left(A\left(F_{T-1}\right)+\eta_{T-1}^{V}\left\|A\left(F_{T-1}\right)\right\| I_{d}\right) u_{T-1}}}, \\
V_{T-1} & =\frac{\eta_{T-1}^{V}\left\|A\left(F_{T-1}\right)\right\|}{\left\|u_{T-1}\right\|^{2}} u_{T-1} u_{T-1}^{\prime} .
\end{aligned}
$$

Unlike Theorem 5, we do not need to consider the case $u_{T-1}=\mathbf{0}_{d}$ since $\left.\mathbf{0}_{d} \notin \widetilde{\mathcal{A}}_{T}^{u}\right|_{T-1} ^{T-1}$. Therefore, the time $T-1$ problem is

$$
\begin{gathered}
\widehat{Y}_{T-1}^{T}=\max _{\left.u_{T-1} \in \widetilde{\mathcal{A}}_{T}^{u}\right|_{T-1} ^{T-1}}\left\{1+\left(m\left(F_{T-1}\right)\right)^{\prime} u_{T-1}-\frac{\widehat{\gamma}_{T-1}}{2} u_{T-1}^{\prime}\left(A\left(F_{T-1}\right)+\eta_{T-1}^{V}\left\|A\left(F_{T-1}\right)\right\| I_{d}\right) u_{T-1}\right. \\
\left.-\eta_{T-1}^{\theta} \sqrt{u_{T-1}^{\prime}\left(A\left(F_{T-1}\right)+\eta_{T-1}^{V}\left\|A\left(F_{T-1}\right)\right\| I_{d}\right) u_{T-1}}\right\}
\end{gathered}
$$

The Lagrange function of the above problem is

$$
\begin{aligned}
\mathcal{L}^{u}= & 1+\left(m\left(F_{T-1}\right)\right)^{\prime} u_{T-1}-\frac{\widehat{\gamma}_{T-1}}{2} u_{T-1}^{\prime}\left(A\left(F_{T-1}\right)+\eta_{T-1}^{V}\left\|A\left(F_{T-1}\right)\right\| I_{d}\right) u_{T-1} \\
& -\eta_{T-1}^{\theta} \sqrt{u_{T-1}^{\prime}\left(A\left(F_{T-1}\right)+\eta_{T-1}^{V}\left\|A\left(F_{T-1}\right)\right\| I_{d}\right) u_{T-1}}+\lambda_{u}\left(1-\mathbf{1}_{d}^{\prime} u_{T-1}\right)
\end{aligned}
$$

where $\lambda_{u}$ is the Lagrange multiplier. The first order condition is

$$
m\left(F_{T-1}\right)-\lambda_{u} \mathbf{1}_{d}-\left(\widehat{\gamma}_{T-1}+\frac{\eta_{T-1}^{\theta}}{\psi_{T-1}^{*}}\right)\left(A\left(F_{T-1}\right)+\eta_{T-1}^{V}\left\|A\left(F_{T-1}\right)\right\| I_{d}\right) u_{T-1}=\mathbf{0}_{d},
$$

where

$$
\psi_{T-1}^{*}=\sqrt{u_{T-1}^{\prime}\left(A\left(F_{T-1}\right)+\eta_{T-1}^{V}\left\|A\left(F_{T-1}\right)\right\| I_{d}\right) u_{T-1}} .
$$


Hence,

$$
u_{T-1}=\left(\widehat{\gamma}_{T-1}+\frac{\eta_{T-1}^{\theta}}{\psi_{T-1}^{*}}\right)^{-1}\left(A\left(F_{T-1}\right)+\eta_{T-1}^{V}\left\|A\left(F_{T-1}\right)\right\| I_{d}\right)^{-1}\left(m\left(F_{T-1}\right)-\lambda_{u} \mathbf{1}_{d}\right) .
$$

Since the constraint is binded, we have

$$
1=\mathbf{1}_{d}^{\prime} u_{T-1}=\left(\widehat{\gamma}_{T-1}+\frac{\eta_{T-1}^{\theta}}{\psi_{T-1}^{*}}\right)^{-1}\left(c_{T-1}^{\text {worf }}-\lambda_{u} b_{T-1}^{\text {worf }}\right) .
$$

Therefore, we obtain

$$
\lambda_{u}=\frac{1}{b_{T-1}^{w o r f}}\left(c_{T-1}^{\text {worf }}-\left(\widehat{\gamma}_{T-1}+\frac{\eta_{T-1}^{\theta}}{\psi_{T-1}^{*}}\right)\right) .
$$

The optimal portfolio is

$$
\begin{aligned}
u_{T-1}=\frac{1}{\widehat{\gamma}_{T-1}+\eta_{T-1}^{\theta} / \psi_{T-1}^{*}} & \left(A\left(F_{T-1}\right)+\eta_{T-1}^{V}\left\|A\left(F_{T-1}\right)\right\| I_{d}\right)^{-1} \\
& \times\left(m\left(F_{T-1}\right)-\frac{c_{T-1}^{\text {worf }}-\left(\widehat{\gamma}_{T-1}+\eta_{T-1}^{\theta} / \psi_{T-1}^{*}\right)}{b_{T-1}^{\text {worf }}} \mathbf{1}_{d}\right) .
\end{aligned}
$$

Then,

$$
\begin{aligned}
& b_{T-1}^{\text {worf }}\left(\psi_{T-1}^{*}\right)^{2} \\
& =b_{T-1}^{\text {worf }} u_{T-1}^{\prime}\left(A\left(F_{T-1}\right)+\eta_{T-1}^{V}\left\|A\left(F_{T-1}\right)\right\| I_{d}\right) u_{T-1} \\
& =\frac{b_{T-1}^{\text {worf }}}{\left(\widehat{\gamma}_{T-1}+\frac{\eta_{T-1}^{\theta}}{\psi_{T-1}^{*}}\right)^{2}}\left(a_{T-1}^{\text {worf }}-2 \frac{c_{T-1}^{\text {worf }}-\left(\widehat{\gamma}_{T-1}+\frac{\eta_{T-1}^{\theta}}{\psi_{T-1}^{*}}\right)}{b_{T-1}^{\text {worf }}} c_{T-1}^{\text {worf }}+\frac{\left.\left(c_{T-1}^{\text {worf }}-\left(\widehat{\gamma}_{T-1}+\frac{\eta_{T-1}^{\theta}}{\psi_{T-1}^{*}}\right)\right)^{2}\right)}{b_{T-1}^{\text {worf }}}\right) \\
& =\frac{b_{T-1}^{\text {worf }}}{\left(\widehat{\gamma}_{T-1}+\frac{\eta_{T-1}^{\theta}}{\psi_{T-1}^{*}}\right)^{2}}\left(a_{T-1}^{\text {worf }}-\frac{\left(c_{T-1}^{\text {worf }}\right)^{2}}{b_{T-1}^{\text {worf }}}+\frac{\left(\widehat{\gamma}_{T-1}+\frac{\eta_{T-1}^{\theta}}{\psi_{T-1}^{*}}\right)^{2}}{b_{T-1}^{\text {worf }}}\right) \\
& =\frac{a_{T-1}^{\text {worf }} b_{T-1}^{\text {worf }}-\left(c_{T-1}^{\text {worf }}\right)^{2}}{\left(\widehat{\gamma}_{T-1}+\frac{\eta_{T-1}^{\theta}}{\psi_{T-1}^{*}}\right)^{2}}+1=\frac{d_{T-1}^{\text {worf }}}{\left(\widehat{\gamma}_{T-1}+\frac{\eta_{T-1}^{\theta}}{\psi_{T-1}^{*}}\right)^{2}}+1 .
\end{aligned}
$$

Therefore, we can consider the following polynomial equation for $\psi_{T-1}^{*}$ :

$$
b_{T-1}^{w o r f}\left(\psi_{T-1}^{*}\right)^{2}=\frac{d_{T-1}^{\text {worf }}}{\left(\widehat{\gamma}_{T-1}+\eta_{T-1}^{\theta} / \psi_{T-1}^{*}\right)^{2}}+1 .
$$

Expanding it, we obtain

$$
\begin{aligned}
q\left(\psi_{T-1}^{*}\right):= & b_{T-1}^{\text {worf }} \widehat{\gamma}_{T-1}^{2}\left(\psi_{T-1}^{*}\right)^{4}+2 b_{T-1}^{\text {worf }} \widehat{\gamma}_{T-1} \eta_{T-1}^{\theta}\left(\psi_{T-1}^{*}\right)^{3} \\
& +\left(b_{T-1}^{\text {worf }}\left(\eta_{T-1}^{\theta}\right)^{2}-\left(d_{T-1}^{\text {worf }}+\widehat{\gamma}_{T-1}^{2}\right)\right)\left(\psi_{T-1}^{*}\right)^{2} \\
& -2 \widehat{\gamma}_{T-1} \eta_{T-1}^{\theta} \psi_{T-1}^{*}-\left(\eta_{T-1}^{\theta}\right)^{2}=0 .
\end{aligned}
$$

The equation $q\left(\psi_{T-1}^{*}\right)=0$ is essentially the same as the equation (A11) in Garlappi et al. (2007), therefore it has a unique positive solution by the discussion in Garlappi et al. (2007). We show the existence of the unique positive solution to the equation (A.9). Since the equation 
(A.9) is a quartic equation, it has four solutions. Since $q(0)=-\left(\eta_{T-1}^{\theta}\right)^{2}<0$ and $b_{T-1}^{\text {worf }} \widehat{\gamma}_{T-1}^{2}>$ 0, the equation (A.9) has at least two real solutions: the one is a positive solution and the one is a negative solution. We show that the positive solution is unique. Consider the following quadratic equation.

$$
\begin{aligned}
\frac{\mathrm{d}^{2} q\left(\psi_{T-1}^{*}\right)}{\mathrm{d}\left(\psi_{T-1}^{*}\right)^{2}}= & 12 b_{T-1}^{\text {worf }} \widehat{\gamma}_{T-1}^{2}\left(\psi_{T-1}^{*}\right)^{2}+12 b_{T-1}^{\text {worf }} \widehat{\gamma}_{T-1} \eta_{T-1}^{\theta} \psi_{T-1}^{*} \\
& +2\left(b_{T-1}^{\text {worf }}\left(\eta_{T-1}^{\theta}\right)^{2}-\left(d_{T-1}^{w o r f}+\widehat{\gamma}_{T-1}^{2}\right)\right)=0 .
\end{aligned}
$$

The discriminant of the quadratic equation (A.10) is

$$
\begin{aligned}
D & =36\left(b_{T-1}^{\text {worf }}\right)^{2} \widehat{\gamma}_{T-1}^{2}\left(\eta_{T-1}^{\theta}\right)^{2}-24 b_{T-1}^{\text {worf }} \widehat{\gamma}_{T-1}^{2}\left(b_{T-1}^{\text {worf }}\left(\eta_{T-1}^{\theta}\right)^{2}-\left(d_{T-1}^{\text {worf }}+\widehat{\gamma}_{T-1}^{2}\right)\right) \\
& =12\left(b_{T-1}^{\text {worf }}\right)^{2} \widehat{\gamma}_{T-1}^{2}\left(\eta_{T-1}^{\theta}\right)^{2}+24 b_{T-1}^{\text {worf }} \widehat{\gamma}_{T-1}^{2}\left(d_{T-1}^{\text {worf }}+\widehat{\gamma}_{T-1}^{2}\right)>0
\end{aligned}
$$

Hence, the equation (A.10) has two real solutions. Moreover, we have

$$
\frac{\mathrm{d}^{2} q\left(\psi_{T-1}^{*}\right)}{\mathrm{d}\left(\psi_{T-1}^{*}\right)^{2}}=12 b_{T-1}^{\text {worf }} \widehat{\gamma}_{T-1}^{2}\left(\psi_{T-1}^{*}+\frac{\eta_{T-1}^{\theta}}{2 \widehat{\gamma}_{T-1}}\right)^{2}-\left(b_{T-1}^{\text {worf }}\left(\eta_{T-1}^{\theta}\right)^{2}+d_{T-1}^{\text {worf }}+\widehat{\gamma}_{T-1}^{2}\right) .
$$

Since $\eta_{T-1}^{\theta} /\left(2 \widehat{\gamma}_{T-1}\right)>0$, the equation (A.10) has at least one negative solution. Next, consider the first derivative of $q$ such that

$$
\begin{aligned}
\frac{\mathrm{d} q\left(\psi_{T-1}^{*}\right)}{\mathrm{d} \psi_{T-1}^{*}}= & 4 b_{T-1}^{\text {worf }} \widehat{\gamma}_{T-1}^{2}\left(\psi_{T-1}^{*}\right)^{3}+6 b_{T-1}^{\text {worf }} \widehat{\gamma}_{T-1} \eta_{T-1}\left(\psi_{T-1}^{*}\right)^{2} \\
& +2\left(b_{T-1}^{\text {worf }}\left(\eta_{T-1}^{\theta}\right)^{2}-\left(d_{T-1}^{\text {worf }}+\widehat{\gamma}_{T-1}^{2}\right)\right) \psi_{T-1}^{*}-2 \widehat{\gamma}_{T-1} \eta_{T-1}^{\theta}=0 .
\end{aligned}
$$

Then, the cubic equation (A.11) has at least one positive solution since $4 b_{T-1}^{\text {worf }} \widehat{\gamma}_{T-1}^{2}>0$ and $-2 \widehat{\gamma}_{T-1} \eta_{T-1}^{\theta}<0$. However, since the equation (A.10) has at least one negative solution, we conclude that the equation (A.11) has a unique positive solution. Now, let us consider the quartic equation (A.9) again. Since $q(0)=-\left(\eta_{T-1}^{\theta}\right)^{2}<0$ and $b_{T-1}^{\text {worf }} \widehat{\gamma}_{T-1}^{2}>0$ hold, and since the equation (A.11) has a unique positive solution, the equation (A.9) has at most one local minimum on $\psi_{T-1}^{*}>0$. Furthermore, we also have

$$
\left.\frac{\mathrm{d} q\left(\psi_{T-1}^{*}\right)}{\mathrm{d} \psi_{T-1}^{*}}\right|_{\psi_{T-1}^{*}=0}=-2 \widehat{\gamma}_{T-1} \eta_{T-1}^{\theta}<0
$$

These facts imply that the equation (A.9) has only one positive solution. Therefore, the positive solution of the equation (A.9) is unique. We also write $\psi_{T-1}^{*}$ as the positive solution of the equation (A.9). Then, the optimal portfolio is

$$
\begin{aligned}
u_{T-1}=\frac{1}{\widehat{\gamma}_{T-1}+\eta_{T-1}^{\theta} / \psi_{T-1}^{*}} & \left(A\left(F_{T-1}\right)+\eta_{T-1}^{V}\left\|A\left(F_{T-1}\right)\right\| I_{d}\right)^{-1} \\
& \times\left(m\left(F_{T-1}\right)-\frac{c_{T-1}^{\text {worf }}-\left(\widehat{\gamma}_{T-1}+\eta_{T-1}^{\theta} / \psi_{T-1}^{*}\right)}{b_{T-1}^{\text {worf }}} \mathbf{1}_{d}\right) \\
=\frac{1}{\widehat{\gamma}_{T-1}+\left|g_{T-1}\right| \eta_{T-1}^{\theta} / \psi_{T-1}^{*}}\left(A\left(F_{T-1}\right)+\eta_{T-1}^{V}\left\|A\left(F_{T-1}\right)\right\| I_{d}\right)^{-1} & \times\left(m_{T-1}-\frac{c_{T-1}^{\text {worf }}-\left(\widehat{\gamma}_{T-1}+\left|g_{T-1}\right| \eta_{T-1}^{\theta} / \psi_{T-1}^{*}\right)}{b_{T-1}^{\text {worf }}} \mathbf{1}_{d}\right),
\end{aligned}
$$


where we have used $g_{T-1}=1$ and $m_{T-1}=m\left(F_{T-1}\right)$. Since the coefficients of the quartic equation (A.9) are $\mathcal{F}_{T-1}^{R, F}$-measurable, its solution $\psi_{T-1}^{*}$ is also $\mathcal{F}_{T-1}^{R, F}$-measurable. Therefore, $u_{T-1}$ is $\mathcal{F}_{T-1}^{R, F}$-measurable. The value function at time $T-1$ is

$$
\begin{aligned}
\widehat{Y}_{T-1}^{T}= & +\left(m\left(F_{T-1}\right)\right)^{\prime} u_{T-1}-\frac{\widehat{\gamma}_{T-1}}{2}\left(\psi_{T-1}^{*}\right)^{2}-\eta_{T-1}^{\theta} \psi_{T-1}^{*} \\
= & g_{T-1}+\frac{1}{\widehat{\gamma}_{T-1}+\left|g_{T-1}\right| \eta_{T-1}^{\theta} / \psi_{T-1}^{*}}\left(a_{T-1}^{\text {worf }}-\frac{c_{T-1}^{\text {worf }}-\left(\widehat{\gamma}_{T-1}+\left|g_{T-1}\right| \eta_{T-1}^{\theta} / \psi_{T-1}^{*}\right)}{b_{T-1}^{w o r f}} c_{T-1}^{\text {worf }}\right) \\
& \quad-\frac{\widehat{\gamma}_{T-1}}{2}\left(\psi_{T-1}^{*}\right)^{2}-\left|g_{T-1}\right| \eta_{T-1}^{\theta} \psi_{T-1}^{*} \\
= & g_{T-1}+\frac{c_{T-1}^{\text {worf }}}{b_{T-1}^{\text {worf }}}+\frac{d_{T-1}^{\text {worf }}}{b_{T-1}^{\text {worf }}\left(\widehat{\gamma}_{T-1}+\left|g_{T-1}\right| \eta_{T-1}^{\theta} / \psi_{T-1}^{*}\right)}-\frac{\widehat{\gamma}_{T-1}}{2}\left(\psi_{T-1}^{*}\right)^{2}-\left|g_{T-1}\right| \eta_{T-1}^{\theta} \psi_{T-1}^{*},
\end{aligned}
$$

where we have used $g_{T-1}=1$. Using the equation (A.8), we have

$$
\begin{aligned}
& \frac{d_{T-1}^{\text {worf }}}{b_{T-1}^{\text {worf }}\left(\widehat{\gamma}_{T-1}+\left|g_{T-1}\right| \eta_{T-1}^{\theta} / \psi_{T-1}^{*}\right)}-\frac{\widehat{\gamma}_{T-1}}{2}\left(\psi_{T-1}^{*}\right)^{2}-\left|g_{T-1}\right| \eta_{T-1}^{\theta} \psi_{T-1}^{*} \\
& =\left(\left(\psi_{T-1}^{*}\right)^{2}-\frac{1}{b_{T-1}^{\text {worf }}}\right)\left(\widehat{\gamma}_{T-1}+\frac{\left|g_{T-1}\right| \eta_{T-1}^{\theta}}{\psi_{T-1}^{*}}\right)-\left(\widehat{\gamma}_{T-1}+\frac{\left|g_{T-1}\right| \eta_{T-1}^{\theta}}{\psi_{T-1}^{*}}\right)\left(\psi_{T-1}^{*}\right)^{2} \\
& \quad+\frac{\widehat{\gamma}_{T-1}}{2}\left(\psi_{T-1}^{*}\right)^{2} \\
& =-\frac{\widehat{\gamma}_{T-1}+\left|g_{T-1}\right| \eta_{T-1}^{\theta} / \psi_{T-1}^{*}}{b_{T-1}^{\text {worf }}}+\frac{\widehat{\gamma}_{T-1}}{2}\left(\psi_{T-1}^{*}\right)^{2} .
\end{aligned}
$$

Hence, the value function is

$$
\widehat{Y}_{T-1}^{T}=g_{T-1}+\frac{c_{T-1}^{\text {worf }}-\left(\widehat{\gamma}_{T-1}+\left|g_{T-1}\right| \eta_{T-1}^{\theta} / \psi_{T-1}^{*}\right)}{b_{T-1}^{\text {worf }}}+\frac{\widehat{\gamma}_{T-1}}{2}\left(\psi_{T-1}^{*}\right)^{2}
$$

and it is $\mathcal{F}_{T-1}^{F}$-measurable.

Now, we assume that $\widehat{Y}_{t+1}^{T}$ is $\mathcal{F}_{t+1}^{F}$-measurable for some $0 \leq t \leq T-2$. Then, we have

$$
\begin{aligned}
& \widehat{c}_{t}\left(F_{t}, \theta_{t}, V_{t}, u_{t}\right)+\delta \mathrm{E}^{\theta, V}\left[Y_{t+1}^{T}\left(1+\left(R_{t+1}\right)^{\prime} u_{t}\right) \mid \mathcal{F}_{t}^{R, S}\right] \\
& =g_{t}+\left(m_{t}+g_{t} \theta_{t}\right)^{\prime} u_{t}-\frac{\widehat{\gamma}_{t}}{2} u_{t}^{\prime}\left(A\left(F_{t}\right)+V_{t}\right) u_{t} .
\end{aligned}
$$

Then, the minimization problem for $\left(\theta_{t}, V_{t}\right)$ can be reduced to

$$
\min _{\left.\left(\theta_{t}, V_{t}\right) \in \mathcal{A}_{T}^{\theta, V}\right|_{t} ^{t}}\left\{g_{t} \theta_{t}^{\prime} u_{t}-\frac{\widehat{\gamma}_{t}}{2} u_{t}^{\prime} V_{t} u_{t}\right\}
$$

Similarly to the time $T-1$ problem, the solution of the above problem is

$$
\begin{aligned}
\theta_{t} & =-\frac{\operatorname{sgn}\left(g_{t}\right) \eta_{t}^{\theta}\left(A\left(F_{t}\right)+\eta_{t}^{V}\left\|A\left(F_{t}\right)\right\| I_{d}\right) u_{t}}{\sqrt{u_{t}^{\prime}\left(A\left(F_{t}\right)+\eta_{t}^{V}\left\|A\left(F_{t}\right)\right\| I_{d}\right) u_{t}}}, \\
V_{t} & =\frac{\eta_{t}^{V}\left\|A\left(F_{t}\right)\right\|}{\left\|u_{t}\right\|^{2}} u_{t} u_{t}^{\prime} .
\end{aligned}
$$


Therefore, the maximization problem for $u_{t}$ is

$$
\begin{array}{r}
\max _{\left.u_{t} \in \widetilde{\mathcal{A}}_{T}\right|_{t} ^{t}}\left\{g_{t}+m_{t}^{\prime} u_{t}-\frac{\widehat{\gamma}_{t}}{2} u_{t}^{\prime}\left(A\left(F_{t}\right)+\eta_{t}^{V}\left\|A\left(F_{t}\right)\right\| I_{d}\right) u_{t}\right. \\
\left.-\left|g_{t}\right| \eta_{t}^{\theta} \sqrt{u_{t}^{\prime}\left(A\left(F_{t}\right)+\eta_{t}^{V}\left\|A\left(F_{t}\right)\right\| I_{d}\right) u_{t}}\right\} .
\end{array}
$$

Since the above problem has the same form as the time $T-1$ problem, the optimal portfolio is

$$
u_{t}=\frac{1}{\widehat{\gamma}_{t}+\left|g_{t}\right| \eta_{t}^{\theta} / \psi_{t}^{*}}\left(A\left(F_{t}\right)+\eta_{t}^{V}\left\|A\left(F_{t}\right)\right\| I_{d}\right)^{-1}\left(m_{t}-\frac{c_{t}^{\text {worf }}-\left(\widehat{\gamma}_{t}+\left|g_{t}\right| \eta_{t}^{\theta} / \psi_{t}^{*}\right)}{b_{t}^{\text {worf }}} \mathbf{1}_{d}\right),
$$

where $\psi_{t}^{*}$ is the unique positive solution of the following quartic equation:

$$
b_{t}^{\text {worf }}\left(\psi_{t}^{*}\right)^{2}=\frac{d_{t}^{\text {worf }}}{\left(\widehat{\gamma}_{t}+\left|g_{t}\right| \eta_{t}^{\theta} / \psi_{t}^{*}\right)^{2}}+1 .
$$

The value function is

$$
\widehat{Y}_{t}^{T}=g_{t}+\frac{c_{t}^{\text {worf }}-\left(\widehat{\gamma}_{t}+\left|g_{t}\right| \eta_{t}^{\theta} / \psi_{t}^{*}\right)}{b_{t}^{\text {worf }}}+\frac{\widehat{\gamma}_{t}}{2}\left(\psi_{t}^{*}\right)^{2}
$$

and it is $\mathcal{F}_{t}^{F}$-measurable.

By the mathematical induction, we therefore conclude that the optimal portfolio and that the value function is determined as in Proposition 7 .

Proof of Proposition 8. In this case, we can also apply the dynamic programming procedure. Consider the time $T-1$ problem,

$$
\begin{aligned}
\widehat{Y}_{T-1}^{+T} & \min _{\left.\left.u_{T-1} \in \widetilde{\mathcal{A}}_{+T}^{u}\right|_{T-1} ^{T-1}\left(\theta_{T-1}, V_{T-1}\right) \in \mathcal{A}_{T}^{\theta, V}\right|_{T-1} ^{T-1}}\left\{\begin{aligned}
1+ & \left(m\left(F_{T-1}\right)+\theta_{T-1}\right)^{\prime} u_{T-1} \\
& \left.-\frac{\widehat{\gamma}_{T-1}}{2} u_{T-1}^{\prime}\left(A\left(F_{T-1}\right)+V_{T-1}\right) u_{T-1}\right\} .
\end{aligned}\right.
\end{aligned}
$$

Since $g_{T-1}^{+}=1$ and $m_{T-1}^{+}=m\left(F_{T-1}\right)$ by the definition, the objective function of the time $T-1$ problem can be expressed as

$$
g_{T-1}^{+}+\left(m_{T-1}^{+}+g_{T-1}^{+} \theta_{T-1}\right)^{\prime} u_{T-1}-\frac{\widehat{\gamma}_{T-1}}{2} u_{T-1}^{\prime}\left(A\left(F_{T-1}\right)+V_{T-1}\right) u_{T-1} .
$$

Hence, it suffices to consider the following optimization problem.

$$
\begin{aligned}
\max _{\left.u_{T-1} \in \widetilde{\mathcal{A}}_{+T}^{u} T_{T-1}^{T-1}\left(\theta_{T-1}, V_{T-1}\right) \in \mathcal{A}_{T}^{\theta, V}\right|_{T-1} ^{T-1}}\left\{g_{T-1}^{+}\right. & +\left(m_{T-1}^{+}+g_{T-1}^{+} \theta_{T-1}\right)^{\prime} u_{T-1} \\
& \left.-\frac{\widehat{\gamma}_{T-1}}{2} u_{T-1}^{\prime}\left(A\left(F_{T-1}\right)+V_{T-1}\right) u_{T-1}\right\} .
\end{aligned}
$$

Solving the inner minimization problem, we can express the above problem as

$$
\begin{array}{r}
\max _{u_{T-1} \in \widetilde{\mathcal{A}}_{+T}^{u} T_{T-1}^{T-1}}\left\{g_{T-1}^{+}+\left(m_{T-1}^{+}\right)^{\prime} u_{T-1}-\frac{\widehat{\gamma}_{T-1}}{2} u_{T-1}^{\prime}\left(A\left(F_{T-1}\right)+\eta_{T-1}^{V}\left\|A\left(F_{T-1}\right)\right\| I_{d}\right) u_{T-1}\right. \\
\left.-\left|g_{T-1}^{+}\right| \eta_{T-1}^{\theta} \sqrt{u_{T-1}^{\prime}\left(A\left(F_{T-1}\right)+\eta_{T-1}^{V}\left\|A\left(F_{T-1}\right)\right\| I_{d}\right) u_{T-1}}\right\} .
\end{array}
$$


Now, we consider the auxiliary problem of (A.12), whose feasible set of portfolios is $\overline{\mathcal{C}^{+}}$. If we regard $\overline{\mathcal{C}^{+}}$as a correspondence from $\mathbb{R}^{K}$ to $\mathbb{R}^{d}$, then its graph is

$$
\operatorname{Gr}\left(\overline{\mathcal{C}^{+}}\right):=\left\{(f, \phi) \in \mathbb{R}^{K} \times \mathbb{R}^{d} \mid \phi \in \overline{\mathcal{C}^{+}}\right\}=\mathbb{R}^{K} \times \overline{\mathcal{C}^{+}} .
$$

It is clear that $\operatorname{Gr}\left(\overline{\mathcal{C}^{+}}\right)$is a Borel subset on $\mathbb{R}^{K} \times \mathbb{R}^{d}$. This implies that $\overline{\mathcal{C}^{+}}$is Borel measurable. Furthermore, the objective function of the problem (A.12) is continuous in $u_{T-1}$ and also $\mathcal{F}_{T-1}^{F}$-measurable for any fixed $u_{T-1}$. Moreover, $\overline{\mathcal{C}^{+}}$is a non-empty compact subset of $\mathbb{R}^{d}$ endowed with the Euclidean topology. Hence, by the measurable selection theorem (see Appendix D in Hernández-Lerma and Lasserre (1996)), there exists an $\mathcal{F}_{T-1}^{F}$-measurable random vector $u_{T-1}^{*} \in \overline{\mathcal{C}^{+}}$such that

$$
\begin{array}{r}
g_{T-1}^{+}+\left(m_{T-1}^{+}\right)^{\prime} u_{T-1}^{*}-\frac{\widehat{\gamma}_{T-1}}{2}\left(u_{T-1}^{*}\right)^{\prime}\left(A\left(F_{T-1}\right)+\eta_{T-1}^{V}\left\|A\left(F_{T-1}\right)\right\| I_{d}\right) u_{T-1}^{*} \\
-\left|g_{T-1}^{+}\right| \eta_{T-1}^{\theta} \sqrt{\left(u_{T-1}^{*}\right)^{\prime}\left(A\left(F_{T-1}\right)+\eta_{T-1}^{V}\left\|A\left(F_{T-1}\right)\right\| I_{d}\right) u_{T-1}^{*}} \\
=\max _{u_{T-1} \in \mathcal{C}^{+}}\left\{g_{T-1}^{+}+\left(m_{T-1}^{+}\right)^{\prime} u_{T-1}-\frac{\widehat{\gamma}_{T-1}}{2} u_{T-1}^{\prime}\left(A\left(F_{T-1}\right)+\eta_{T-1}^{V}\left\|A\left(F_{T-1}\right)\right\| I_{d}\right) u_{T-1}\right. \\
\left.-\left|g_{T-1}^{+}\right| \eta_{T-1}^{\theta} \sqrt{u_{T-1}^{\prime}\left(A\left(F_{T-1}\right)+\eta_{T-1}^{V}\left\|A\left(F_{T-1}\right)\right\| I_{d}\right) u_{T-1}}\right\} .
\end{array}
$$

Moreover, since $\left.u_{T-1}^{*} \in \widetilde{\mathcal{A}}_{+T}^{u}\right|_{T-1} ^{T-1}$ and $\left.\widetilde{\mathcal{A}}_{+T}^{u}\right|_{T-1} ^{T-1} \subseteq \overline{\mathcal{C}^{+}}$, we conclude that

$$
\begin{aligned}
& g_{T-1}^{+}+\left(m_{T-1}^{+}\right)^{\prime} u_{T-1}^{*}-\frac{\widehat{\gamma}_{T-1}}{2}\left(u_{T-1}^{*}\right)^{\prime}\left(A\left(F_{T-1}\right)+\eta_{T-1}^{V}\left\|A\left(F_{T-1}\right)\right\| I_{d}\right) u_{T-1}^{*} \\
& -\left|g_{T-1}^{+}\right| \eta_{T-1}^{\theta} \sqrt{\left(u_{T-1}^{*}\right)^{\prime}\left(A\left(F_{T-1}\right)+\eta_{T-1}^{V}\left\|A\left(F_{T-1}\right)\right\| I_{d}\right) u_{T-1}^{*}} \\
& =\max _{\left.u_{T-1} \in \widetilde{\mathcal{A}}_{+T}^{u}\right|_{T-1} ^{T-1}}\left\{g_{T-1}^{+}+\left(m_{T-1}^{+}\right)^{\prime} u_{T-1}-\frac{\widehat{\gamma}_{T-1}}{2} u_{T-1}^{\prime}\left(A\left(F_{T-1}\right)+\eta_{T-1}^{V}\left\|A\left(F_{T-1}\right)\right\| I_{d}\right) u_{T-1}\right. \\
& \left.-\left|g_{T-1}^{+}\right| \eta_{T-1}^{\theta} \sqrt{u_{T-1}^{\prime}\left(A\left(F_{T-1}\right)+\eta_{T-1}^{V}\left\|A\left(F_{T-1}\right)\right\| I_{d}\right) u_{T-1}}\right\} \\
& =\max _{u_{T-1} \in \overline{\mathcal{C}^{+}}}\left\{g_{T-1}^{+}+\left(m_{T-1}^{+}\right)^{\prime} u_{T-1}-\frac{\widehat{\gamma}_{T-1}}{2} u_{T-1}^{\prime}\left(A\left(F_{T-1}\right)+\eta_{T-1}^{V}\left\|A\left(F_{T-1}\right)\right\| I_{d}\right) u_{T-1}\right. \\
& \left.-\left|g_{T-1}^{+}\right| \eta_{T-1}^{\theta} \sqrt{u_{T-1}^{\prime}\left(A\left(F_{T-1}\right)+\eta_{T-1}^{V}\left\|A\left(F_{T-1}\right)\right\| I_{d}\right) u_{T-1}}\right\} \\
& =\widehat{Y}_{T-1}^{+T}
\end{aligned}
$$

This implies that $u_{T-1}^{*}$ is the maximizer of the problem (A.12). Moreover, $\widehat{Y}_{T-1}^{+T}$ is $\mathcal{F}_{T-1^{-}}^{F}$ measurable by the measurable selection theorem.

Now, we hypothesize that at some time $t+1,0 \leq t+1 \leq T-1, \widehat{Y}_{t+1}^{+T}$ is $\mathcal{F}_{t+1}^{S}$-measurable. 
Then, we have

$$
\begin{aligned}
& \widehat{Y}_{t}^{+T}:=\max _{\left.\left.u_{t} \in \widetilde{\mathcal{A}}_{+T}^{u}\right|_{t} ^{t}\left(\theta_{t}, V_{t}\right) \in \mathcal{A}_{T}^{\theta, V}\right|_{t} ^{t}}\left\{1+\left(m\left(F_{t}\right)+\theta_{t}\right)^{\prime} u_{t}-\frac{\widehat{\gamma}_{t}}{2} u_{t}^{\prime}\left(A\left(F_{t}\right)+V_{t}\right) u_{t}\right. \\
& \left.+\delta \mathrm{E}^{\theta, V}\left[\widehat{Y}_{t+1}^{+T} \mid \mathcal{F}_{t}^{R, F}\right]\right\} \\
& =\max _{\left.\left.u_{t} \in \widetilde{\mathcal{A}}_{+T}^{u}\right|_{t} ^{t}\left(\theta_{t}, V_{t}\right) \in \mathcal{A}_{T}^{\theta, V}\right|_{t} ^{t}}\left\{g_{t}^{+}+\left(m_{t}^{+}+g_{t}^{+} \theta_{t}\right)^{\prime} u_{t}-\frac{\widehat{\gamma}_{t}}{2} u_{t}^{\prime}\left(A\left(F_{t}\right)+V_{t}\right) u_{t}\right\} \\
& =\max _{\left.u_{t} \in \widehat{\mathcal{A}}_{+T}^{u}\right|_{t} ^{t}}\left\{g_{t}^{+}+\left(m_{t}^{+}\right)^{\prime} u_{t}-\frac{\widehat{\gamma}_{t}}{2} u_{t}^{\prime}\left(A\left(F_{t}\right)+\eta_{t}^{V}\left\|A\left(F_{t}\right)\right\| I_{d}\right) u_{t}\right. \\
& \left.-\left|g_{t}^{+}\right| \eta_{t}^{\theta} \sqrt{u_{t}^{\prime}\left(A\left(F_{t}\right)+\eta_{t}^{V}\left\|A\left(F_{t}\right)\right\| I_{d}\right) u_{t}}\right\} \text {. }
\end{aligned}
$$

The problem (A.13) has the same form as the problem (A.12), and $m_{t}^{+}, g_{t}^{+}, \widehat{\gamma}_{t}, A\left(F_{t}\right), \eta_{t}^{\theta}$ and $\eta_{t}^{V}$ are $\mathcal{F}_{t}^{F}$-measurable. Therefore, we can use the measurable selection theorem in the same manner as we did in the case of the problem (A.12). By the measurable selection theorem, there exists an $\mathcal{F}_{t}^{F}$-measurable random vector $u_{t}^{*} \in \overline{\mathcal{C}^{+}}$such that

$$
\begin{gathered}
g_{t}^{+}+\left(m_{t}^{+}\right)^{\prime} u_{t}^{*}-\frac{\widehat{\gamma}_{t}}{2}\left(u_{t}^{*}\right)^{\prime}\left(A\left(F_{t}\right)+\eta_{t}^{V}\left\|A\left(F_{t}\right)\right\| I_{d}\right) u_{t}^{*} \\
-\left|g_{t}^{+}\right| \eta_{t}^{\theta} \sqrt{\left(u_{t}^{*}\right)^{\prime}\left(A\left(F_{t}\right)+\eta_{t}^{V}\left\|A\left(F_{t}\right)\right\| I_{d}\right) u_{t}^{*}} \\
=\max _{\left.u_{t} \in \widetilde{\mathcal{A}}_{+T}^{u}\right|_{t} ^{t}}\left\{g_{t}^{+}+\left(m_{t}^{+}\right)^{\prime} u_{t}-\frac{\widehat{\gamma}_{t}}{2} u_{t}^{\prime}\left(A\left(F_{t}\right)+\eta_{t}^{V}\left\|A\left(F_{t}\right)\right\| I_{d}\right) u_{t}\right. \\
\left.-\left|g_{t}^{+}\right| \eta_{t}^{\theta} \sqrt{u_{t}^{\prime}\left(A\left(F_{t}\right)+\eta_{t}^{V}\left\|A\left(F_{t}\right)\right\| I_{d}\right) u_{t}}\right\} \\
=\max _{u_{t} \in \overline{\mathcal{C}^{+}}}\left\{g_{t}^{+}+\left(m_{t}^{+}\right)^{\prime} u_{t}-\frac{\widehat{\gamma}_{t}}{2} u_{t}^{\prime}\left(A\left(F_{t}\right)+\eta_{t}^{V}\left\|A\left(F_{t}\right)\right\| I_{d}\right) u_{t}\right. \\
\left.-\left|g_{t}^{+}\right| \eta_{t}^{\theta} \sqrt{u_{t}^{\prime}\left(A\left(F_{t}\right)+\eta_{t}^{V}\left\|A\left(F_{t}\right)\right\| I_{d}\right) u_{t}}\right\}=\widehat{Y}_{t}^{+T} .
\end{gathered}
$$

Moreover, $\widehat{Y}_{t}^{+T}$ is $\mathcal{F}_{t}^{F}$-measurable.

By the mathematical induction, $\widehat{Y}_{t}^{+T}$ is $\mathcal{F}_{t}^{F}$-measurable for all $0 \leq t \leq T-1$. Therefore, the value function of the original optimization problem is

$$
V_{+T}^{w o r f}(x, f)=\mathrm{E}\left[\widehat{Y}_{t}^{+T} \mid F_{0}=f\right] x,
$$

and the optimal portfolio at time $t$ is $u_{t}^{*}$.

\section{References}

Ang, A., and G. Bekaert. 2002. International Asset Allocation with Regime Shifts. Review of Financial Studies 15:1137-1187. URL http://dx.doi.org/10.1093/rfs/15.4.1137.

Best, M. J., and R. R. Grauer. 1991. On the Sensitivity of Mean-Variance-Efficient Portfolios to Changes in Asset Means: Some Analytical and Computational Results. Review of Financial Studies 4:315-342. URL http://dx.doi.org/10.1093/rfs/4.2.315. 
Björk, T., A. Murgoci, and X. Y. Zhou. 2014. Mean-Variance Portfolio Optimization with State-Dependent Risk Aversion. Mathematical Finance 24:1-24. URL http://dx.doi.org/10.1111/j.1467-9965.2011.00515.x.

Black, F., and R. Litterman. 1990. Asset Allocation: Combining Investor Views with Market Equilibrium. Discussion Paper, Goldman, Sachs \& Co .

Chen, Z. P., G. Li, and Y. G. Zhao. 2014. Time-Consistent Investment Policies in Markovian Markets: A Case of Mean-Variance Analysis. Journal of Economic Dynamics and Control 40:293-316. URL http://dx.doi.org/10.1016/j.jedc.2014.01.011.

DeMiguel, V., L. Garlappi, F. J. Nogales, and R. Uppal. 2009a. A Generalized Approach to Portfolio Optimization: Improving Performance by Constraining Portfolio Norms. Management Science 55:798-812. URL http://dx.doi.org/10.1287/mnsc.1080.0986.

DeMiguel, V., L. Garlappi, and R. Uppal. 2009b. Optimal Versus Naive Diversification: How Inefficient Is the 1/N Portfolio Strategy? Review of Financial Studies 22:1915-1953. URL http://dx.doi.org/10.1093/rfs/hhm075.

Ellsberg, D. 1961. Risk, Ambiguity, and the Savage Axioms. Quarterly Journal of Economics 75:643-669. URL http://dx.doi.org/10.2307/1884324.

Fama, E. F., and K. R. French. 1993. Common Risk Factors in the Returns on Stocks and Bonds. Journal of Financial Economics 33:3-56. URL http://dx.doi.org/10.1016/0304-405X (93)90023-5.

Garlappi, L., R. Uppal, and T. Wang. 2007. Portfolio Selection with Parameter and Model Uncertainty: A Multi-Prior Approach. Review of Financial Studies 20:41-81. URL http://dx.doi.org/10.1093/rfs/hhl003.

Gilboa, I., and D. Schmeidler. 1989. Maxmin Expected Utility with NonUnique Prior. Journal of Mathematical Economics 18:141-153. URL http://dx.doi.org/10.1016/0304-4068(89)90018-9.

Goldfarb, D., and G. Iyengar. 2003. Robust Portfolio Selection Problems. Mathematics of Operations Research 28:1-38. URL http://dx.doi.org/10.1287/moor.28.1.1.14260.

Hamilton, J. D. 1989. A New Approach to the Economic Analysis of Nonstationary Time Series and the Business Cycle. Econometrica 57:357-384. URL http://dx.doi.org/10.2307/1912559.

Hamilton, J. D. 1990. Analysis of Time Series Subject to Changes in Regime. Journal of Econometrics 45:39-70. URL http://dx.doi.org/10.1016/0304-4076(90)90093-9.

Hernández-Lerma, O., and J. B. Lasserre. 1996. Discrete-Time Markov Control Processes: Basic Optimality Criteria, vol. 30. Springer Science \& Business Media.

Jagannathan, R., and T. Ma. 2003. Risk Reduction in Large Portfolios: Why Imposing the Wrong Constraints Helps. The Journal of Finance 58:1651-1683. URL http://dx.doi.org/10.1111/1540-6261.00580.

Jobson, J. D., and B. Korkie. 1980. Estimation for Markowitz Efficient Portfolios. Journal of the American Statistical Association 75:544-554. URL http://dx.doi.org/10.1080/01621459.1980.10477507. 
Jorion, P. 1986. Bayes-Stein Estimation for Portfolio Analysis. Journal of Financial and Quantitative Analysis 21:279-292. URL http://dx.doi.org/10.2307/2331042.

Ledoit, O., and M. Wolf. 2003. Improved Estimation of the Covariance Matrix of Stock Returns with an Application to Portfolio Selection. Journal of Empirical Finance 10:603621. URL http://dx.doi.org/10.1016/S0927-5398(03)00007-0.

Ledoit, O., and M. Wolf. 2004. A Well-Conditioned Estimator for LargeDimensional Covariance Matrices. Journal of Multivariate Analysis 88:365-411. URL http://dx.doi.org/10.1016/S0047-259X (03) 00096-4.

Li, D., and W.-L. Ng. 2000. Optimal Dynamic Portfolio Selection: Multiperiod Mean-Variance Formulation. Mathematical Finance 10:387-406. URL http://dx.doi.org/10.1111/1467-9965.00100.

Markowitz, H. 1952. Portfolio Selection. The Journal of Finance 7:77-91. URL http://dx.doi.org/10.1111/j.1540-6261.1952.tb01525.x.

Merton, R. C. 1972. An Analytic Derivation of the Efficient Portfolio Frontier. Journal of Financial and Quantitative Analysis 7:1851-1872. URL http://dx.doi.org/10.2307/2329621.

Merton, R. C. 1980. On Estimating the Expected Return on the Market: An Exploratory Investigation. Journal of Financial Economics 8:323-361. URL http://dx.doi.org/10.1016/0304-405X (80)90007-0.

Pástor, L., and R. F. Stambaugh. 2000. Comparing Asset Pricing Models: An Investment Perspective. Journal of Financial Economics 56:335-381. URL http://dx.doi.org/10.1016/S0304-405X(00) 00044-1.

Pflug, G. C., A. Pichler, and D. Wozabal. 2012. The 1/N Investment Strategy Is Optimal under High Model Ambiguity. Journal of Banking and Finance 36:410-417. URL http://dx.doi.org/10.1016/j.jbankfin.2011.07.018.

Pınar, M. Ç. 2014. Equilibrium in an Ambiguity-Averse Mean-Variance Investors Market. European Journal of Operational Research 237:957-965. URL http://dx.doi.org/10.1016/j.ejor.2014.02.016.

Sharpe, W. F. 1964. Capital Asset Prices: A Theory of Market Equilibrium under Conditions of Risk. The Journal of Finance 19:425-442. URL http://dx.doi.org/10.1111/j.1540-6261.1964.tb02865.x.

Tobin, J. 1958. Liquidity Preference As Behavior Towards Risk. The Review of Economic Studies 25:65-86. URL http://dx.doi.org/10.2307/2296205.

Wozabal, D. 2014. Robustifying Convex Risk Measures for Linear Portfolios: A Nonparametric Approach. Operations Research 62:1302-1315. URL http://dx.doi.org/10.1287/opre.2014.1323. 
Table 1. The Abbreviations of Typical Portfolios and Six Extreme Strategies. Panel A displays the abbreviations of typical portfolios, and Panel B displays the details concerning the extreme strategies. The first column in Panel B lists the abbreviations of the extreme strategies. The second and third columns represent portfolios in different states. The forth and fifth columns display behaviors of parameters which justify the extreme strategies.

Panel A

Abbreviation

Portfolio

$\max \mathrm{SR}$

The portfolio maximizing the empirical Sharpe ratio

GMV

EW

VW

The single-period global minimum-variance portfolio

The equally weighted portfolio

The value-weighted portfolio

NE (No Error) The no error portfolio (always $\eta_{t}^{\theta}=\eta_{t}^{V}=0$ )

Panel B

\begin{tabular}{ccccc}
\multirow{2}{*}{ Abbreviation } & \multicolumn{2}{c}{ Portfolio } & \multicolumn{2}{c}{ Parameters } \\
& Good State & Bad State & Good State & Bad State \\
\hline GMV-EW & GMV & EW & $\eta_{t}^{V}=0$ and $\eta_{t}^{\theta} \rightarrow \infty$ & $\eta_{t}^{V} \rightarrow \infty$ \\
EW-NE & EW & No Error & $\eta_{t}^{V} \rightarrow \infty$ & $\eta_{t}^{\theta}=\eta_{t}^{V}=0$ \\
GMV-NE & GMV & No Error & $\eta_{t}^{V}=0$ and $\eta_{t}^{\theta} \rightarrow \infty$ & $\eta_{t}^{\theta}=\eta_{t}^{V}=0$ \\
EW-GMV & EW & GMV & $\eta_{t}^{V} \rightarrow \infty$ & $\eta_{t}^{V}=0$ and $\eta_{t}^{\theta} \rightarrow \infty$ \\
NE-EW & No Error & EW & $\eta_{t}^{\theta}=\eta_{t}^{V}=0$ & $\eta_{t}^{V} \rightarrow \infty$ \\
NE-GMV & No Error & GMV & $\eta_{t}^{\theta}=\eta_{t}^{V}=0$ & $\eta_{t}^{V}=0$ and $\eta_{t}^{\theta} \rightarrow \infty$ \\
\hline
\end{tabular}


Table 2. International Diversification $(d=4)$. This table reports the Sharpe ratios of various strategies obtained from the back test of the international asset allocations. "IID" represents the investor who believes that the number of market states is 1 . "RS" represents the investor who believes that the number of market states is 2 . The abbreviations of portfolios are listed in Table 1. In the case of $\eta_{t}^{\theta}=0$ with short selling, we can not compute the optimal portfolios since they diverge. Therefore, we use the portfolios of $\eta_{t}^{\theta}=1$ and $\eta_{t}^{V}=0$ as the No Error portfolios in the extreme strategies.

Short selling is permitted.

IID

\begin{tabular}{ccccccccc} 
& & \multicolumn{9}{c}{$\eta_{t}^{\theta}$} & & GMV \\
$\max \mathrm{SR}$ & & 0.0 & 1.0 & 2.0 & 3.0 & 4.0 & 5.0 & $\infty$ \\
\cline { 3 - 9 } 0.1014 & 0.0 & - & 0.0954 & 0.0941 & 0.0936 & 0.0934 & 0.0933 & 0.0927 \\
& 0.5 & - & 0.0878 & 0.0872 & 0.0869 & 0.0868 & 0.0867 & 0.0864 \\
$\eta_{t}^{V}$ & 1.0 & - & 0.0849 & 0.0843 & 0.0842 & 0.0841 & 0.0840 & 0.0838 \\
& 3.0 & - & 0.0818 & 0.0815 & 0.0814 & 0.0814 & 0.0813 & 0.0812 \\
& 5.0 & - & 0.0810 & 0.0808 & 0.0807 & 0.0807 & 0.0807 & 0.0806 \\
\hline
\end{tabular}

RS

\begin{tabular}{ccccccccc} 
& & \multicolumn{9}{c}{$\eta_{t}^{\theta}$} \\
$\max \mathrm{SR}$ & & 0.0 & 1.0 & 2.0 & 3.0 & 4.0 & 5.0 & \multicolumn{1}{c}{ GMV } \\
\cline { 3 - 9 } 0.0889 & 0.0 & - & 0.0837 & 0.0855 & 0.0859 & 0.0860 & 0.0861 & 0.0863 \\
& 0.5 & - & 0.0856 & 0.0852 & 0.0850 & 0.0849 & 0.0849 & 0.0847 \\
$\eta_{t}^{V}$ & 1.0 & - & 0.0835 & 0.0831 & 0.0830 & 0.0829 & 0.0829 & 0.0827 \\
& 3.0 & - & 0.0813 & 0.0811 & 0.0810 & 0.0809 & 0.0809 & 0.0808 \\
& 5.0 & - & 0.0808 & 0.0805 & 0.0805 & 0.0804 & 0.0804 & 0.0803 \\
\hline
\end{tabular}

Short selling is not permitted.

IID

\begin{tabular}{ccccccccc} 
& & \multicolumn{9}{c}{$\eta_{t}^{\theta}$} & & & GMV \\
$\max \mathrm{SR}$ & & 0.0 & 1.0 & 2.0 & 3.0 & 4.0 & 5.0 & $\infty$ \\
\cline { 3 - 10 } 0.1014 & 0.0 & 0.1025 & 0.1028 & 0.1021 & 0.1018 & 0.1017 & 0.1016 & 0.1012 \\
& 0.5 & 0.0896 & 0.0878 & 0.0872 & 0.0869 & 0.0868 & 0.0867 & 0.0864 \\
$\eta_{t}^{V}$ & 1.0 & 0.1078 & 0.0849 & 0.0843 & 0.0842 & 0.0841 & 0.0840 & 0.0838 \\
& 3.0 & 0.1019 & 0.0818 & 0.0815 & 0.0814 & 0.0814 & 0.0813 & 0.0812 \\
& 5.0 & 0.1081 & 0.0810 & 0.0808 & 0.0807 & 0.0807 & 0.0807 & 0.0806 \\
\hline
\end{tabular}

RS

\begin{tabular}{|c|c|c|c|c|c|c|c|c|}
\hline \multirow{2}{*}{$\max \mathrm{SR}$} & \multirow[b]{3}{*}{00} & \multicolumn{6}{|c|}{$\eta_{t}^{\theta}$} & \multirow{2}{*}{$\begin{array}{c}\text { GMV } \\
\infty\end{array}$} \\
\hline & & 0.0 & 1.0 & 2.0 & 3.0 & 4.0 & 5.0 & \\
\hline 0.0889 & & 0.0597 & 0.0973 & 0.0952 & 0.0942 & 0.0937 & 0.0934 & 0.0920 \\
\hline \multirow{4}{*}{$\eta_{t}^{V}$} & 0.5 & 0.0674 & 0.0856 & 0.0852 & 0.0850 & 0.0849 & 0.0849 & 0.0847 \\
\hline & 1.0 & 0.0583 & 0.0835 & 0.0831 & 0.0830 & 0.0829 & 0.0829 & 0.0827 \\
\hline & 3.0 & 0.0577 & 0.0813 & 0.0811 & 0.0810 & 0.0809 & 0.0809 & 0.0808 \\
\hline & 5.0 & 0.0570 & 0.0808 & 0.0805 & 0.0805 & 0.0804 & 0.0804 & 0.0803 \\
\hline
\end{tabular}

EW 0.0795
Short selling is permitted.

$\begin{array}{ccc}\text { GMV-EW } & \text { EW-NE } & \text { GMV-NE } \\ 0.1032 & 0.0664 & 0.0891 \\ \text { EW-GMV } & \text { NE-EW } & \text { NE-GMV } \\ 0.0636 & 0.0976 & 0.0809\end{array}$

Extreme Strategies

Short selling is not permitted.

GMV-EW EW-NE GMV-NE

$\begin{array}{lll}0.0922 & 0.0949 & 0.1078\end{array}$

EW-GMV NE-EW NE-GMV

$\begin{array}{lll}0.0783 & 0.0457 & 0.0433\end{array}$


Table 3. Diversification across Industries $(d=5)$. This table reports the Sharpe ratios of various strategies obtained from the back test of the asset allocations across industries. "IID" represents the investor who believes that the number of market states is 1. "RS" represents the investor who believes that the number of market states is 2 . The abbreviations of portfolios are listed in Table 1. In the case of $\eta_{t}^{\theta}=0$ with short selling, we can not compute the optimal portfolios since they diverge. Therefore, we use the portfolios of $\eta_{t}^{\theta}=1$ and $\eta_{t}^{V}=0$ as the No Error portfolios in the extreme strategies.

Short selling is permitted.

IID

\begin{tabular}{ccccccccc} 
& & \multicolumn{9}{c}{$\eta_{t}^{\theta}$} & & & GMV \\
$\max \mathrm{SR}$ & & 0.0 & 1.0 & 2.0 & 3.0 & 4.0 & 5.0 & $\infty$ \\
\cline { 3 - 9 } 0.2210 & 0.0 & - & 0.2732 & 0.2791 & 0.2808 & 0.2817 & 0.2821 & 0.2839 \\
& 0.5 & - & 0.2213 & 0.2220 & 0.2222 & 0.2224 & 0.2224 & 0.2227 \\
$\eta_{t}^{V}$ & 1.0 & - & 0.2165 & 0.2169 & 0.2170 & 0.2171 & 0.2171 & 0.2173 \\
& 3.0 & - & 0.2127 & 0.2129 & 0.2129 & 0.2130 & 0.2130 & 0.2131 \\
& 5.0 & - & 0.2119 & 0.2120 & 0.2121 & 0.2121 & 0.2121 & 0.2121 \\
\hline
\end{tabular}

RS

\begin{tabular}{ccccccccc} 
& & \multicolumn{9}{c}{$\eta_{t}^{\theta}$} & & & GMV \\
$\max \mathrm{SR}$ & & 0.0 & 1.0 & 2.0 & 3.0 & 4.0 & 5.0 & $\infty$ \\
\cline { 3 - 9 } 0.1968 & 0.0 & - & 0.1912 & 0.2524 & 0.2601 & 0.2632 & 0.2649 & 0.2707 \\
& 0.5 & - & 0.2238 & 0.2269 & 0.2278 & 0.2283 & 0.2286 & 0.2297 \\
$\eta_{t}^{V}$ & 1.0 & - & 0.2181 & 0.2202 & 0.2209 & 0.2212 & 0.2214 & 0.2222 \\
& 3.0 & - & 0.2129 & 0.2141 & 0.2145 & 0.2147 & 0.2148 & 0.2153 \\
& 5.0 & - & 0.2118 & 0.2127 & 0.2130 & 0.2131 & 0.2132 & 0.2136 \\
\hline
\end{tabular}

Short selling is not permitted.

\begin{tabular}{|c|c|c|c|c|c|c|c|c|}
\hline & & & & & & & & GMV \\
\hline $\max S R$ & & 0.0 & 1.0 & 2.0 & 3.0 & 4.0 & 5.0 & $\infty$ \\
\hline \multirow[t]{2}{*}{0.2210} & 0.0 & 0.1822 & 0.2496 & 0.2547 & 0.2561 & 0.2570 & 0.2576 & 0.2596 \\
\hline & 0.5 & 0.1643 & 0.2213 & 0.2220 & 0.2222 & 0.2224 & 0.2224 & 0.2227 \\
\hline \multirow{3}{*}{$\eta_{t}^{V}$} & 1.0 & 0.1724 & 0.2165 & 0.2169 & 0.2170 & 0.2171 & 0.2171 & 0.2173 \\
\hline & 3.0 & 0.1783 & 0.2127 & 0.2129 & 0.2129 & 0.2130 & 0.2130 & 0.2131 \\
\hline & 5.0 & 0.1487 & 0.2119 & 0.2120 & 0.2121 & 0.2121 & 0.2121 & 0.2121 \\
\hline
\end{tabular}

RS

\begin{tabular}{|c|c|c|c|c|c|c|c|c|}
\hline \multirow{2}{*}{$\max \mathrm{SR}$} & \multirow[b]{3}{*}{00} & \multicolumn{6}{|c|}{$\eta_{t}^{\theta}$} & \multirow{2}{*}{$\begin{array}{c}\text { GMV } \\
\infty\end{array}$} \\
\hline & & 0.0 & 1.0 & 2.0 & 3.0 & 4.0 & 5.0 & \\
\hline \multirow[t]{2}{*}{0.1968} & & 0.0660 & 0.2394 & 0.2502 & 0.2543 & 0.2573 & 0.2592 & 0.2664 \\
\hline & 0.5 & 0.0796 & 0.2239 & 0.2269 & 0.2278 & 0.2283 & 0.2286 & 0.2297 \\
\hline \multirow[t]{3}{*}{$\eta_{t}^{V}$} & 1.0 & 0.0769 & 0.2181 & 0.2202 & 0.2209 & 0.2212 & 0.2214 & 0.2222 \\
\hline & 3.0 & 0.0653 & 0.2129 & 0.2141 & 0.2145 & 0.2147 & 0.2148 & 0.2153 \\
\hline & 5.0 & 0.0841 & 0.2118 & 0.2127 & 0.2130 & 0.2131 & 0.2132 & 0.2136 \\
\hline
\end{tabular}

EW

0.0795

VW
0.1825

Short selling is permitted.

GMV-EW EW-NE GMV-NE

$\begin{array}{lll}0.2234 & 0.2457 & 0.2592\end{array}$

EW-GMV NE-EW NE-GMV $\begin{array}{lll}0.2564 & 0.1657 & 0.1964\end{array}$
Extreme Strategies

Short selling is not permitted.

GMV-EW EW-NE GMV-NE

$\begin{array}{lll}0.2281 & 0.1199 & 0.1344\end{array}$

EW-GMV NE-EW NE-GMV

$\begin{array}{lll}0.2473 & 0.1410 & 0.1678\end{array}$


Table 4. Diversification among Sizes and Values $(d=6)$. This table reports the Sharpe ratios of various strategies obtained from the back test of the asset allocations among the $2 \times 3$ size- and book-to-market-sorted portfolios by Fama and French (1993). "IID" represents the investor who believes that the number of market states is 1 . "RS" represents the investor who believes that the number of market states is 2 . The abbreviations of portfolios are listed in Table 1 . In the case of $\eta_{t}^{\theta}=0$ with short selling, we can not compute the optimal portfolios since they diverge. Therefore, we use the portfolios of $\eta_{t}^{\theta}=1$ and $\eta_{t}^{V}=0$ as the No Error portfolios in the extreme strategies.

Short selling is permitted.

IID

\begin{tabular}{ccccccccc} 
& & \multicolumn{9}{c}{$\eta_{t}^{\theta}$} & & & GMV \\
$\max \mathrm{SR}$ & & 0.0 & 1.0 & 2.0 & 3.0 & 4.0 & 5.0 & $\infty$ \\
\cline { 2 - 9 } 0.2139 & 0.0 & - & 0.3406 & 0.3293 & 0.3243 & 0.3215 & 0.3197 & 0.3122 \\
& 0.5 & - & 0.2237 & 0.2228 & 0.2224 & 0.2223 & 0.2222 & 0.2218 \\
$\eta_{t}^{V}$ & 1.0 & - & 0.2176 & 0.2170 & 0.2168 & 0.2167 & 0.2166 & 0.2164 \\
& 3.0 & - & 0.2126 & 0.2123 & 0.2122 & 0.2122 & 0.2121 & 0.2120 \\
& 5.0 & - & 0.2115 & 0.2113 & 0.2112 & 0.2111 & 0.2111 & 0.2110 \\
\hline
\end{tabular}

RS

\begin{tabular}{ccccccccc} 
& & \multicolumn{9}{c}{$\eta_{t}^{\theta}$} \\
$\max \mathrm{SR}$ & & 0.0 & 1.0 & 2.0 & 3.0 & 4.0 & 5.0 & \multicolumn{1}{c}{ GMV } \\
\cline { 3 - 9 } 0.2081 & 0.0 & - & 0.2389 & 0.2473 & 0.2486 & 0.2489 & 0.2491 & 0.2488 \\
& 0.5 & - & 0.2184 & 0.2195 & 0.2198 & 0.2200 & 0.2200 & 0.2203 \\
$\eta_{t}^{V}$ & 1.0 & - & 0.2147 & 0.2154 & 0.2156 & 0.2157 & 0.2158 & 0.2160 \\
& 3.0 & - & 0.2113 & 0.2117 & 0.2118 & 0.2119 & 0.2119 & 0.2120 \\
& 5.0 & - & 0.2105 & 0.2108 & 0.2109 & 0.2109 & 0.2110 & 0.2110 \\
\hline
\end{tabular}

Short selling is not permitted.

IID

\begin{tabular}{ccccccccc} 
& & \multicolumn{9}{c}{$\eta_{t}^{\theta}$} & & GMV \\
$\max \mathrm{SR}$ & & 0.0 & 1.0 & 2.0 & 3.0 & 4.0 & 5.0 & $\infty$ \\
\cline { 3 - 9 } 0.2139 & 0.0 & 0.2202 & 0.2260 & 0.2263 & 0.2255 & 0.2253 & 0.2257 & 0.2256 \\
& 0.5 & 0.2289 & 0.2237 & 0.2228 & 0.2224 & 0.2223 & 0.2222 & 0.2218 \\
$\eta_{t}^{V}$ & 1.0 & 0.2207 & 0.2176 & 0.2170 & 0.2168 & 0.2167 & 0.2166 & 0.2164 \\
& 3.0 & 0.2271 & 0.2126 & 0.2123 & 0.2122 & 0.2122 & 0.2121 & 0.2120 \\
& 5.0 & 0.2291 & 0.2115 & 0.2113 & 0.2112 & 0.2111 & 0.2111 & 0.2110 \\
\hline
\end{tabular}

RS

\begin{tabular}{ccccccccc} 
& & \multicolumn{9}{c}{$\eta_{t}^{\theta}$} \\
$\max \mathrm{SR}$ & & 0.0 & 1.0 & 2.0 & 3.0 & 4.0 & 5.0 & GMV \\
\cline { 3 - 10 } 0.2081 & 0.0 & 0.1791 & 0.2101 & 0.2133 & 0.2146 & 0.2155 & 0.2158 & 0.2175 \\
& 0.5 & 0.1874 & 0.2184 & 0.2195 & 0.2198 & 0.2199 & 0.2200 & 0.2203 \\
$\eta_{t}^{V}$ & 1.0 & 0.1949 & 0.2147 & 0.2154 & 0.2156 & 0.2157 & 0.2158 & 0.2160 \\
& 3.0 & 0.1856 & 0.2113 & 0.2117 & 0.2118 & 0.2119 & 0.2119 & 0.2120 \\
& 5.0 & 0.1999 & 0.2105 & 0.2108 & 0.2109 & 0.2109 & 0.2110 & 0.2110 \\
\hline
\end{tabular}

EW

0.0795

$\begin{array}{cccc} & \text { GMV-EW } & \text { EW-NE } & \text { GMV-NE } \\ \text { VW } & 0.2763 & 0.1697 & 0.2281 \\ 0.2010 & \text { EW-GMV } & \text { NE-EW } & \text { NE-GMV } \\ & 0.1876 & 0.2861 & 0.2592\end{array}$

Extreme Strategies

Short selling is not permitted.

GMV-EW EW-NE GMV-NE

$\begin{array}{ccc}0.2336 & 0.1790 & 0.1979 \\ \text { EW-GMV } & \text { NE-EW } & \text { NE-GMV } \\ 0.1939 & 0.2069 & 0.1920\end{array}$


Table 5. The Results of the Mimicking Strategies' Sharpe Ratios Obtained from the Back Tests. The second and third columns display parameters in the good state, and the fourth and fifth columns display parameters in the bad state. The sixth column shows whether short selling is permitted or not. The seventh column reports the Sharpe ratios of the mimicking extreme strategies. The eighth column reports the Sharpe ratios of the original extreme strategies. The ninth column reports the average root square errors between the original extreme strategies and the mimicking strategies. The extreme strategies are listed in Table 1.

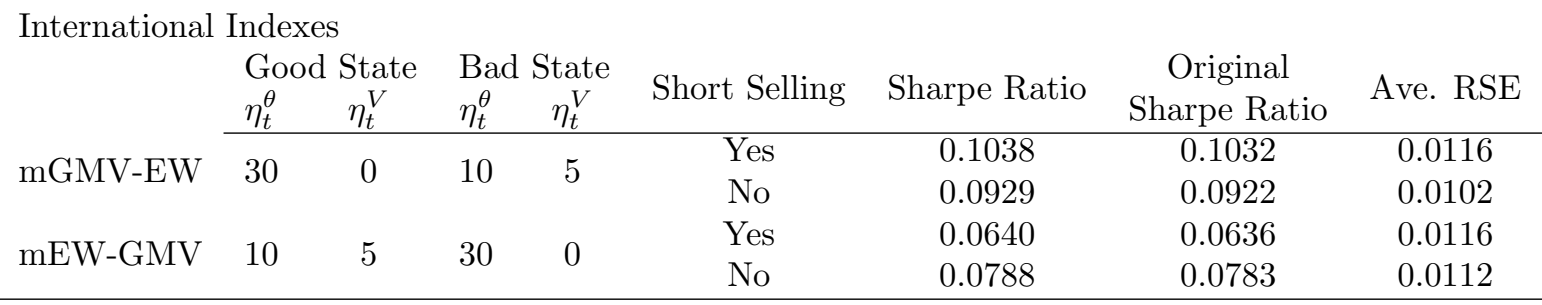

Industry Indexes

\begin{tabular}{|c|c|c|c|c|c|c|c|c|}
\hline & $\begin{array}{l}\text { Goo } \\
\eta_{t}^{\theta}\end{array}$ & $\begin{array}{l}\text { State } \\
\eta_{t}^{V}\end{array}$ & $\begin{array}{l}\mathrm{Bac} \\
\eta_{t}^{\theta}\end{array}$ & $\begin{array}{l}\text { thate } \\
\eta_{t}^{V}\end{array}$ & Short Selling & Sharpe Ratio & $\begin{array}{c}\text { Original } \\
\text { Sharpe Ratio }\end{array}$ & Ave. RSE \\
\hline \multirow{2}{*}{ mGMV-EW } & \multirow{2}{*}{30} & \multirow{2}{*}{0} & \multirow{2}{*}{10} & \multirow{2}{*}{5} & Yes & 0.2252 & 0.2234 & 0.0134 \\
\hline & & & & & No & 0.2303 & 0.2281 & 0.0089 \\
\hline \multirow{2}{*}{ mEW-GMV } & \multirow{2}{*}{10} & \multirow{2}{*}{5} & \multirow{2}{*}{30} & \multirow[b]{2}{*}{0} & Yes & 0.2567 & 0.2564 & 0.0095 \\
\hline & & & & & No & 0.2470 & 0.2473 & 0.0079 \\
\hline
\end{tabular}

Size- and Value-Sorted Portfolios

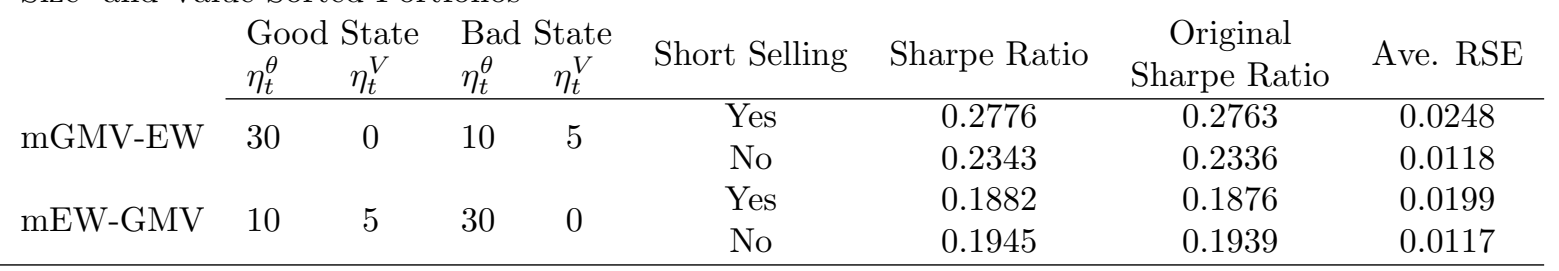


Figure 1. Portfolio Errors of International Indexes Data Set When Short Selling Is Permitted. The following four figures display portfolio errors $\left\|u_{t}^{*}\right\|_{G M V}$ and $\left\|u_{t}^{*}\right\|_{E W}$ at each time $t$. The upper two figures show the portfolio errors from GMV, $\left\|u_{t}^{*}\right\|_{G M V}$, with $\eta_{t}^{V}=0$. The lower two figures represent the portfolio errors from $\mathrm{EW},\left\|u_{t}^{*}\right\|_{E W}$, with $\eta_{t}^{\theta}=5$. The left two figures represent the portfolio errors of the IID investor and the right two figures show those of the RS investor.

Portfolio Errors between GMV and the Optimal Portfolio

IID

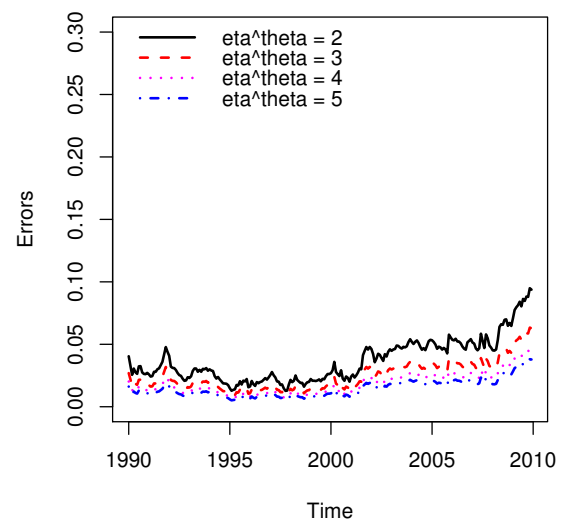

RS

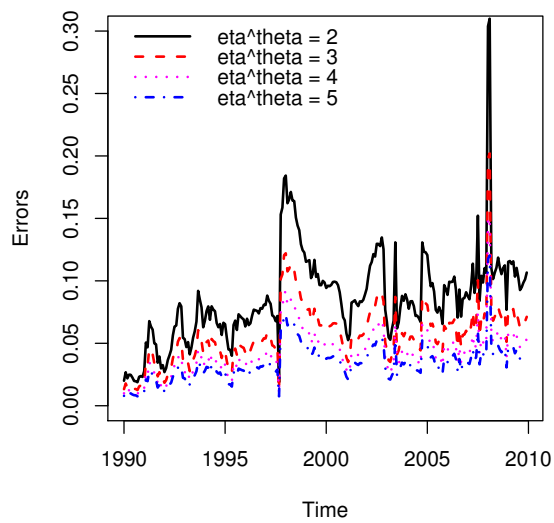

Portfolio Errors between EW and the Optimal Portfolio

IID

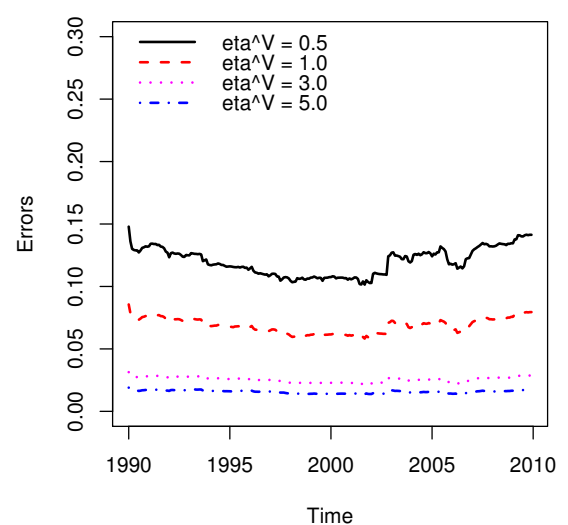

RS

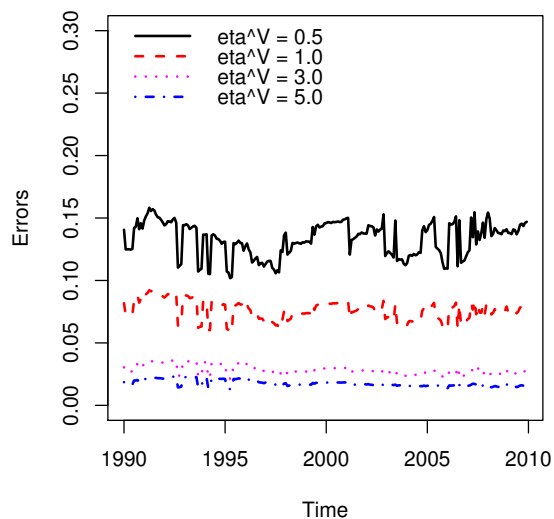

